\title{
VOLTAGE STABILITY ANALYSIS USING SIMULATED SYNCHROPHASOR MEASUREMENTS
}

\author{
A Thesis \\ presented to \\ the Faculty of California Polytechnic State University, \\ San Luis Obispo
}

\author{
In Partial Fulfillment \\ of the Requirements for the Degree \\ Master of Science in Electrical Engineering
}

by

Allan Agatep

May 2013 
(C) 2013

Allan Agatep

\section{ALL RIGHTS RESERVED}




\title{
COMMITTEE MEMBERSHIP
}

TITLE: VOLTAGE STABILITY ANALYSIS USING SIMULATED SYNCHROPHASOR MEASUREMENTS

\author{
AUTHOR: \\ Allan Agatep \\ DATE SUBMITTED: \\ May 2013 \\ COMMITTEE CHAIR: $\quad$ Dr. Ahmad Nafisi, Professor \\ Electrical Engineering Department
}

COMMITTEE MEMBER: Dr. Taufik, Professor Electrical Engineering Department

COMMITTEE MEMBER: Dr. Ali O. Shaban, Professor Electrical Engineering Department 


\section{ABSTRACT \\ VOLTAGE STABILITY ANALYSIS USING SIMULATED SYNCHROPHASOR MEASUREMENTS}

\section{Allan Agatep}

An increase in demand for electric power has forced utility transmission systems to continuously operate under stressed conditions, which are close to instability limits. Operating power systems under such conditions along with inadequate reactive power reserves initiates a sequence of voltage instability points and can ultimately lead to a system voltage collapse. Significant research have been focused on time-synchronized measurements of power systems which can be used to frequently determine the state of a power system and can lead to a more robust protection, control and operation performance. This thesis discusses the applicability of two voltage stability synchrophasor-based indices from literature to analyze the stability of a power system. Various load flow scenarios were conducted on the BPA 10-Bus system and the IEEE 39Bus System using PowerWorld Simulator. The two indices were analyzed and compared against each other along with other well-known methods. Results show that their performances are coherent to each other regarding to voltage stability of the system; the indices can also predict voltage collapse as well as provide insight on other locations within the system that can contribute to instability.

Keywords: voltage stability, voltage stability index, synchrophasors 


\section{ACKNOWLEDGMENTS}

First and foremost, I would like to express my deepest gratitude to my advisor Dr. Ahmad Nafisi for his wisdom and encouragement. This thesis would not have been possible to accomplish without the help and guidance he has provided me throughout the entire process.

I would like to thank Dr. Taufik and Dr. Ali Shaban for serving as members on my thesis committee. I would also like to thank them for their lectures they teach. Their knowledge has been a great resource, which has initially inspired me to pursue power electronics and power systems engineering in the first place.

I would also like to express my dearest appreciation to my friends and fellow colleagues, especially: Abby Cansanay, Alex Morales, Chris Nguyen, Joanne Cho, Mason Ung, Nelson Lau and M.S Rithy Chhean. The discussions and times I've shared with them have helped me get through the most challenging times. Thank you for providing me with most valuable advice and motivation.

Last but not least, I would like to thank my family - especially my parents Noel and Laureen Agatep. My family has always guided me along the right path and has provided me with the inspiration to accomplish great things. They have always been there to help me in any way they can. For all these reasons and more, I am truly grateful. 


\section{TABLE OF CONTENTS}

$\begin{array}{lll}\text { Title } & \text { Page }\end{array}$

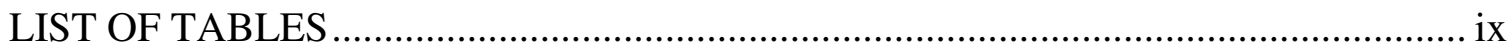

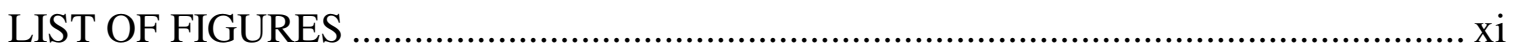

CHAPTER 1 INTRODUCTION..................................................................... 1

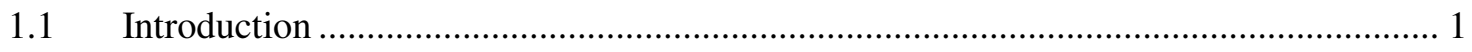

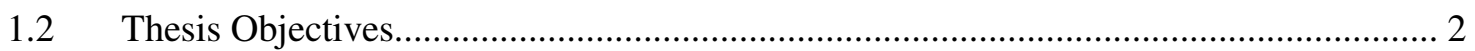

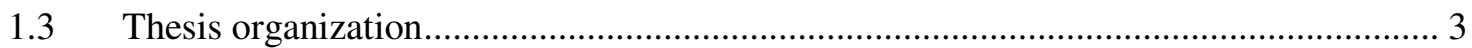

CHAPTER 2 VOLTAGE STABILITY OVERVIEW ........................................... 4

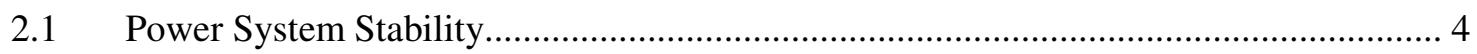

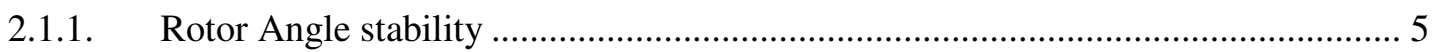

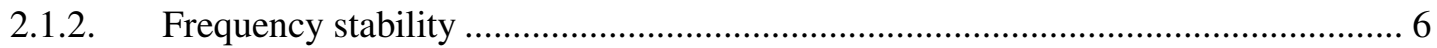

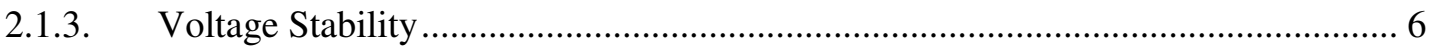

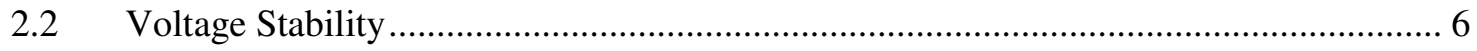

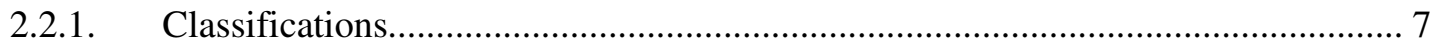

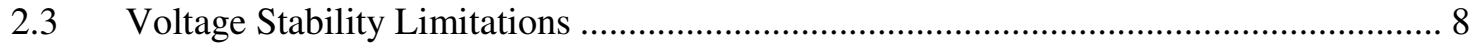

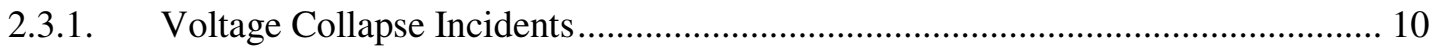

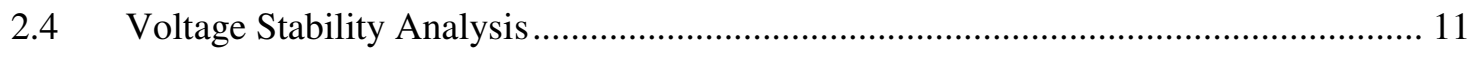

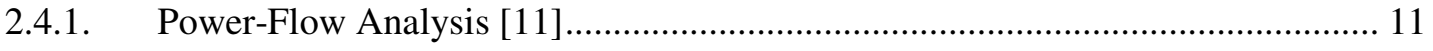

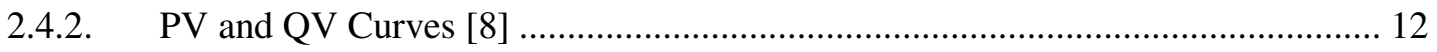

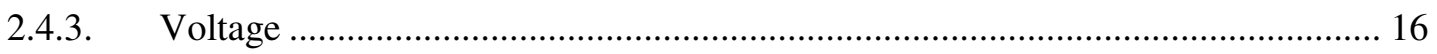

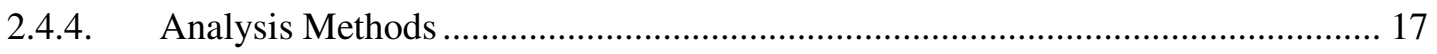

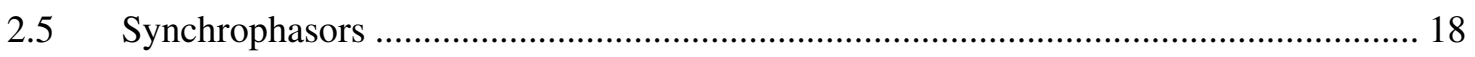

CHAPTER 3 EVALUATION OF VOLTAGE STABILITY INDICES .................... 23

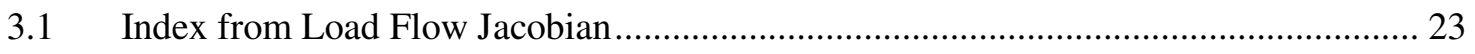

3.1.1. Modal Analysis of Power Flow Model............................................................ 23 
3.2 Indices Based on Phasor Measurement Units.............................................................. 29

3.2.1. Algorithm for Equating Thevenin Equivalent Parameters [26-27] ...................... 30

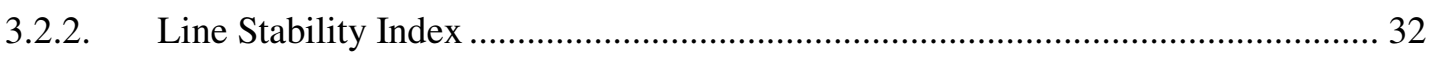

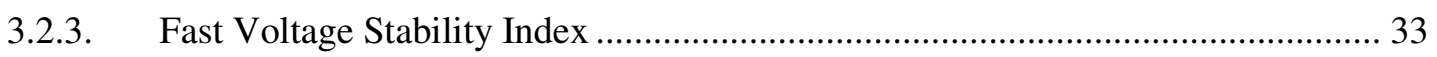

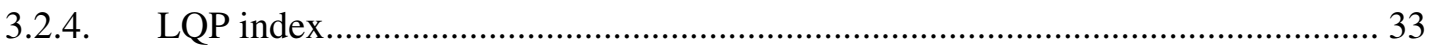

3.2.5. VSI based on Maximum Power Transfers.............................................................. 34

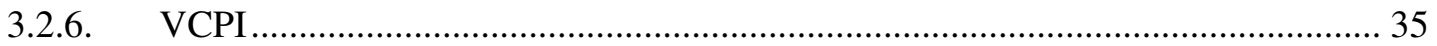

CHAPTER 4 IMPLEMENTATION OF INDICES AND TEST NETWORKS........... 36

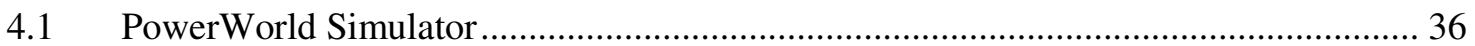

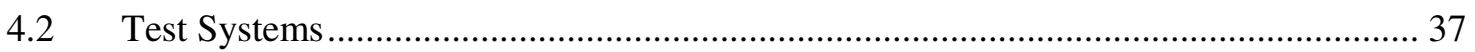

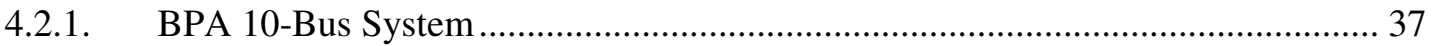

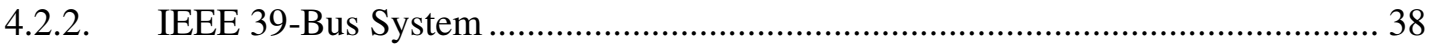

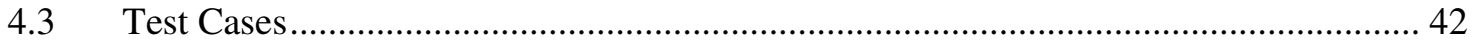

4.3.1. Verification of Index Performance Using 10-Bus Case .................................... 42

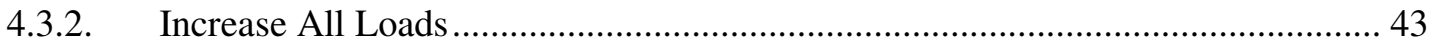

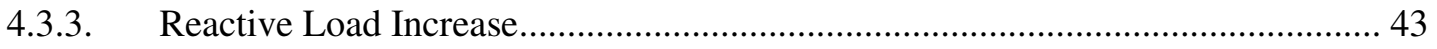

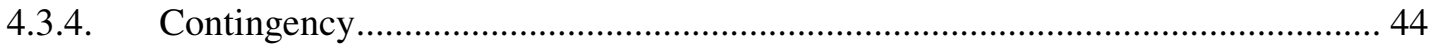

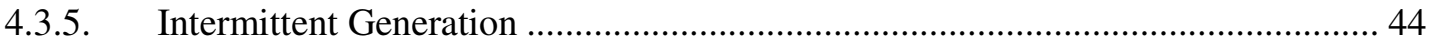

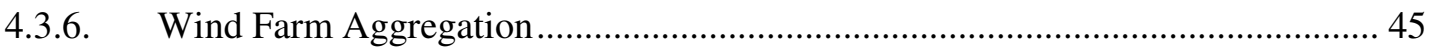

CHAPTER 5 SIMULATION AND RESULTS ..................................................... 47

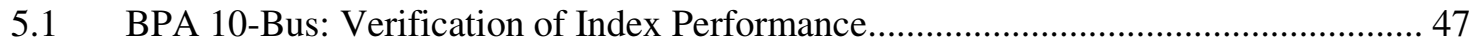

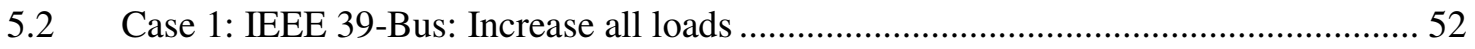

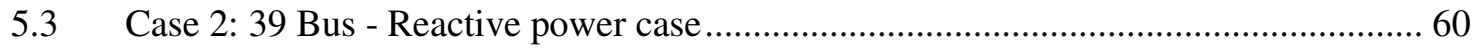

5.3.1. Voltage Collapse Predictability From Indices ...................................................... 64

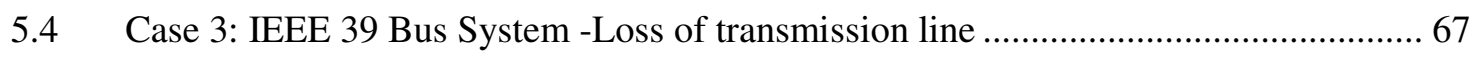

5.5 Case 4: IEEE 39 Bus System - Intermittent Generation .......................................... 76 


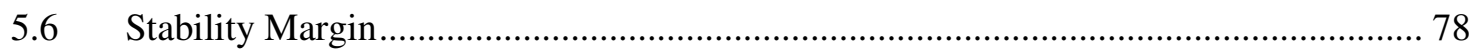

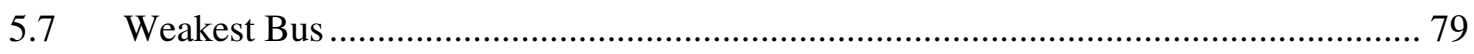

CHAPTER 6 CONCLUSION AND RECOMMENDATIONS ................................ 83

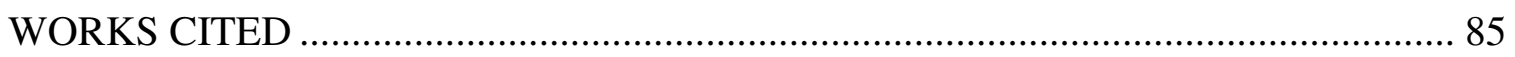

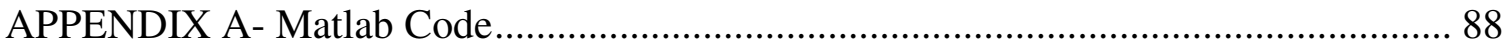

APPENDIX B- Power Flow and Line Loses for Case 3 ........................................... 95

APPENDIX C- QV Analysis Calculations for IEEE-39 Bus System ............................ 96

APPENDIX D- Modal Analysis Calculations for IEEE-39 Bus System ........................ 97 


\section{LIST OF TABLES}

Table

Page

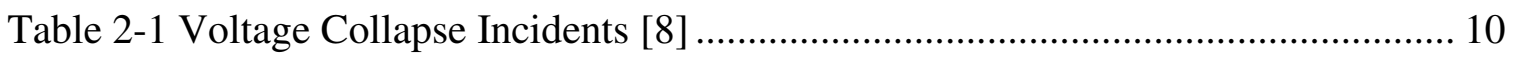

Table 3-1: Eigenvalues for a given load increase ................................................... 27

Table 3-2 Participation Factors For Each Modal Variation (Initial Conditions) .............. 28

Table 3-3 Participation Factors For Each Modal Variation (Last Increase Before

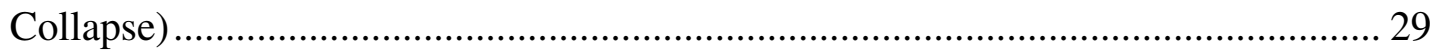

Table 4-1 IEEE 39 Bus Parameters- Load and Generation .......................................... 40

Table 4-2: IEEE 39 Bus Parameters- Line Data .......................................................... 41

Table 4-3 IEEE 39 Bus Parameters- Generator Data..................................................... 42

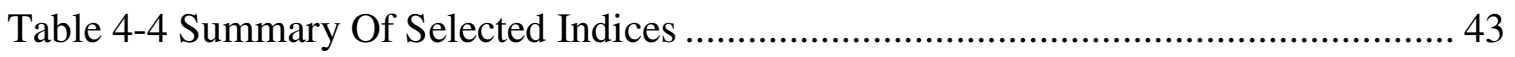

Table 5-1 Load Factor with Corresponding Total System Laod .................................. 52

Table 5-2: Load Bus VSI for Various Load Factors ................................................... 53

Table 5-3 Load Bus VCPI for Various Load Factors .............................................. 54

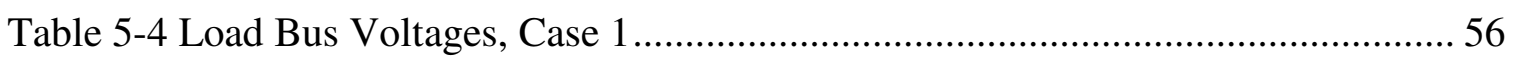

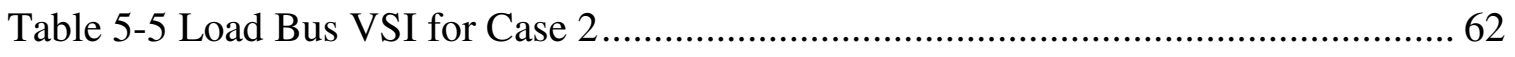

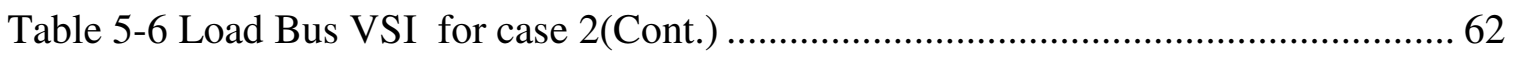

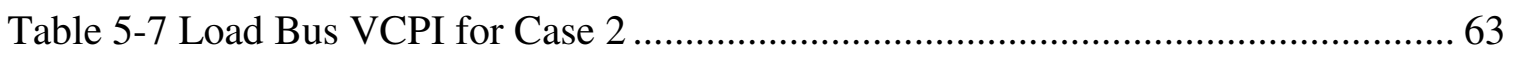

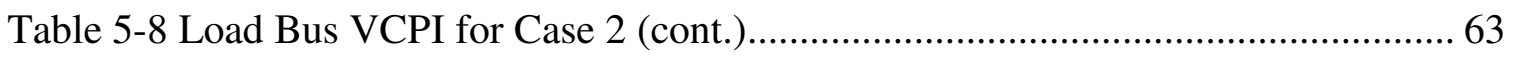

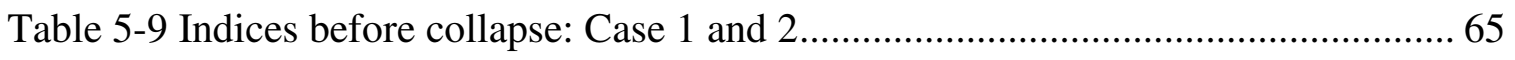

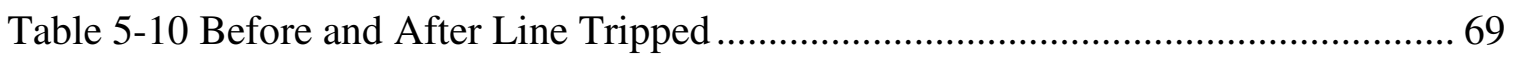

Table 5-11 Load Bus Indices for Case 3, before and after collapse ............................. 71

Table 5-12 Power Flow and Line Losses: Before and After Line Trip ......................... 72 
Table 5-13 Generation and Loads before and after Line Trip

Table 5-14 Line Losses Before and After Line Trip........................................................ 75

Table 5-15 Comparison Of Rankings Between Various Methods: First Load Increase... 80

Table 5-16 Comparison Of Rankings Between Various Methods: Final Load Increase.. 81 


\section{LIST OF FIGURES}

Figure

Page

Figure 2.1 Power System Stability Classifications [8] ............................................ 5

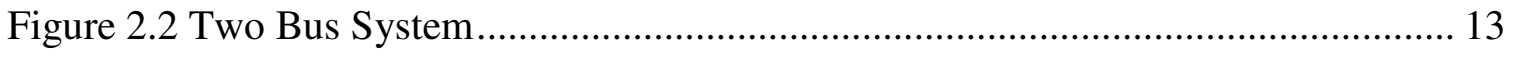

Figure 2.3 PV curves for corresponding power factors: (1) $\varphi=45^{\circ}$ lagging, (2) $\varphi=30^{\circ}$

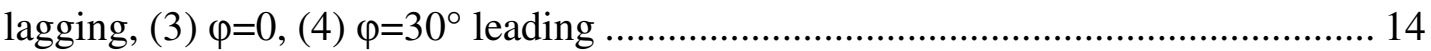

Figure 2.4 QV curves for various load levels. [13] ............................................... 15

Figure 2.5 Convention for Synchrophasor Representation [14] ................................. 19

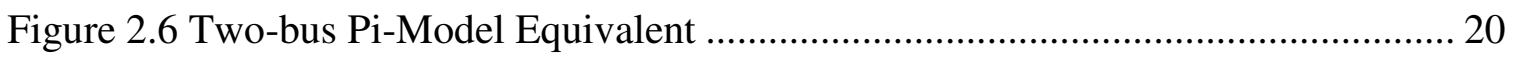

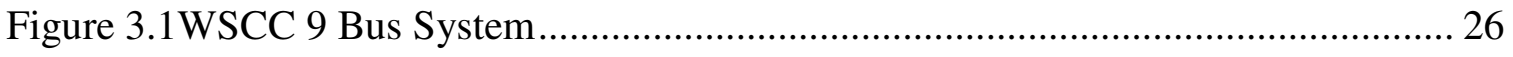

Figure 3.2 Three Lowest Eigenvalues Under System Load Changes........................... 28

Figure 3.3 Thevenin Equivalent with coupling and open circuit voltage ....................... 31

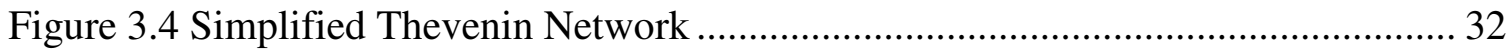

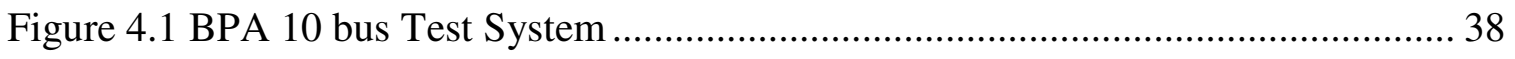

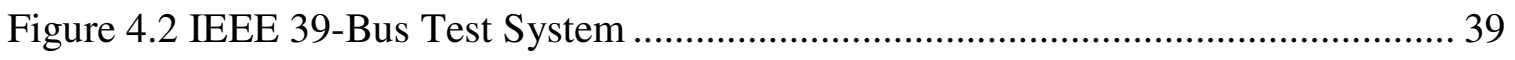

Figure 5.1 Algorithm for Computing VCPI....................................................... 48

Figure 5.2 Algorithm for Computing VSI …..................................................... 49

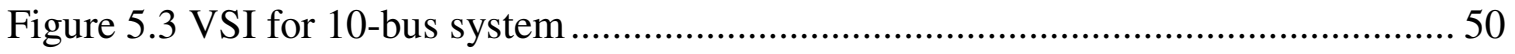

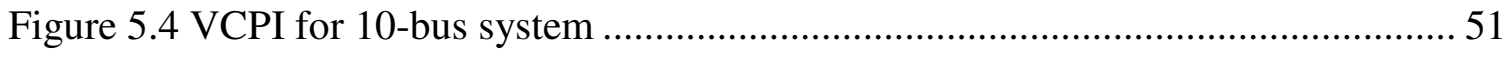

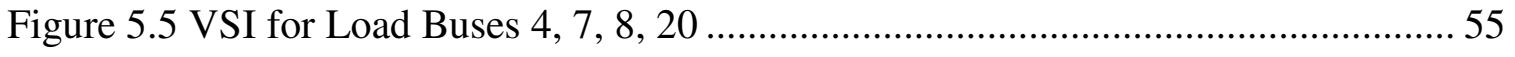

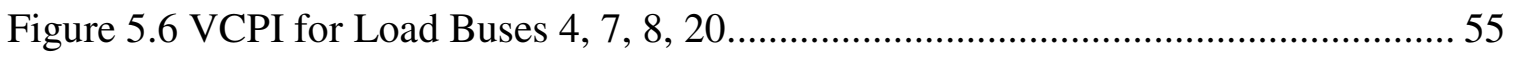

Figure 5.7 Load Buses with respect to nearest generation units ................................ 57

Figure 5.8 VSI and Their Respected Bus Voltages ................................................. 59 
Figure 5.9 VCPI and Their Respected Bus Voltages...............................................5 59

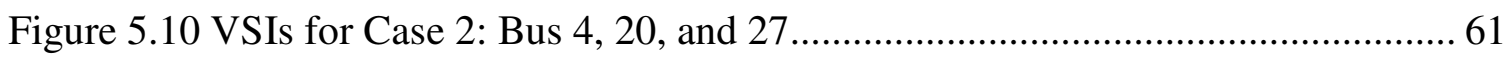

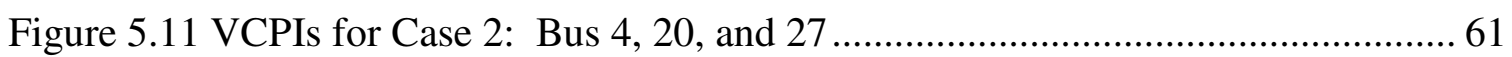

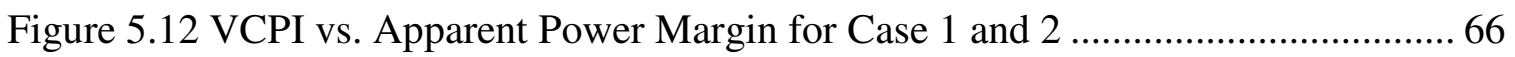

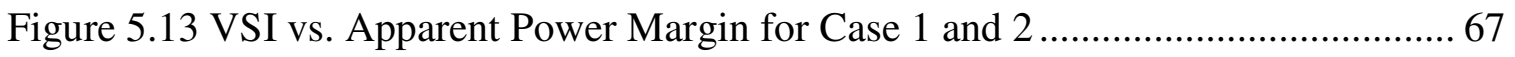

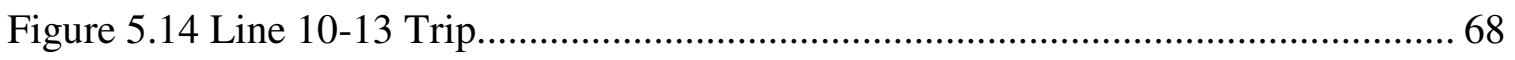

Figure 5.15 VSIs for Case 3: Bus 4, 6, 8, 12 and Global........................................... 70

Figure 5.16 VCPIs for Case 3: Bus 4, 6, 8, 12 and Global ........................................ 70

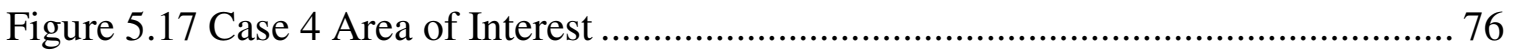

Figure 5.18 Bus 18 VSI for various Wind Generation output .................................... 77

Figure 5.19 Bus 18 VCPI for various Wind Generation output ..................................... 78 


\section{CHAPTER 1 \\ INTRODUCTION}

\subsection{Introduction}

As a result of recent increases in demand for electric power, utility transmission systems have been forced to operate under stressful conditions, often close to instability limits. Efforts to construct new transmission lines or enlarge networks are limited due to economic and environmental constraints. According to U.S. Department of Energy, since 1982, the growth in peak demand for electricity has exceeded transmission growth by almost $25 \%$ every year. The deregulation of electricity market has resulted in increased bulk power across interconnected systems. In some utilities, the amount of transactions previously purchased in a year is now managed in one day [1]. Operating power systems under such conditions along with inadequate reactive power reserves initiates a sequence of voltage instability points and can ultimately lead to a system voltage collapse.

Special attention is being paid to determine methods for assessing voltage stability in real time and developing strategies to mitigate instability issues once they have been detected. Synchronized phasor measurement technology, which is already available at most substation location through protection relays for instance, is capable of directly measuring power system variables (voltage and current phasors) in real time, synchronized to within a millisecond. Together with the improvements on high-speed communication infrastructures, it is possible to build wide area measurement and protection systems [2] to complement classic protection, supervisory control and data acquisition (SCADA), Energy Management Systems (EMS) applications and to 
prevent cascading system level outages. With this new direction on wide area measurement systems, come new approaches for wide area protection and control functions including generating indices for voltage collapse prevention. There are many studies on voltage stability indices including those based on phasor measurements. Some comparisons between these different indices can be found in the literature, such as [3-6]

\subsection{Thesis Objectives}

The main objective of this thesis is to investigate the voltage stability phenomenon using indices based on simulated synchrophasor data. The analysis will consider proximity to voltage collapse: "How close is the system to voltage instability?" and mechanism of voltage instability: "What are the voltage-weak areas?"

In consideration of these questions, this thesis will begin with a discussion on conventional and newer voltage stability methods presented in literature. Next, an analysis and comparison of two indices based on synchrophasor data using static analysis will be conducted and discussed. Various cases, such as increasing system load/generation and/or N-1 contingency will be created to demonstrate the application of indices in voltage stability analysis. Results will reveal their overall effectiveness in its application to the voltage stability problem. An application of these analyses is then briefly discussed in an investigation on the impact of wind generation on voltage stability considering the intermittent nature of wind generation and penetration level. The studied indices will be used in a case where a modeled wind farm is placed on a bus. Indices will be generated for various test cases. Lastly, an analysis will be conducted to demonstrate their significance in the voltage stability problem. Studies and analysis will employ use 
of PowerWorld Software for load flow simulation and Matlab as a post processor tool to calculate indices.

\subsection{Thesis organization}

Chapter 1 introduces the motivation and purpose of this thesis. A survey of literature on the topic of voltage stability indices is briefly discussed and identified. An aim and an appropriate research method are formulated.

Chapter 2 presents the theoretical concepts of voltage stability. A basic overview on power system stability is given before dedicating the remainder of the chapter to explaining voltage stability and methods to analyze the phenomenon.

Chapter 3 explains various indices proposed in literature and their methods used to evaluate the voltage stability of power systems.

Chapter 4 provides a detailed description of two different test networks. The methodology to demonstrate the predictive ability of two indices are formulated

In Chapter 5, the indices chosen are applied according to the methodology from Chapter 4. The results are analyzed and discussed to verify the proposed indices on their applicability to determine voltage stability.

Concluding the thesis will be chapter 6 . A review of the results and its implications are stated with recommendations for future work. 


\section{CHAPTER 2 VOLTAGE STABILITY OVERVIEW}

An overview on power system stability which is based on [7] will be given to provide a global perspective before voltage stability is defined.

\subsection{Power System Stability}

Power system stability is the ability of an electric power system, for a given initial operating condition, to regain a state of operating equilibrium after being subjected to a physical disturbance, with most system variables bounded so that the system remains intact. Due to the various types of disturbances introduced, power system stability can be further classified into appropriate categories: Rotor angle, frequency, and voltage stability. These system variables are categorized, as shown in figure 2.1 , based on disturbance magnitude and time response. In any given situation, one form of instability can possibly lead to another form. However, distinguishing between different forms can provide convenience in identifying the underlying causes of instability, applying an appropriate analysis and ultimately taking corrective measures to return the system to a stable operating point. 


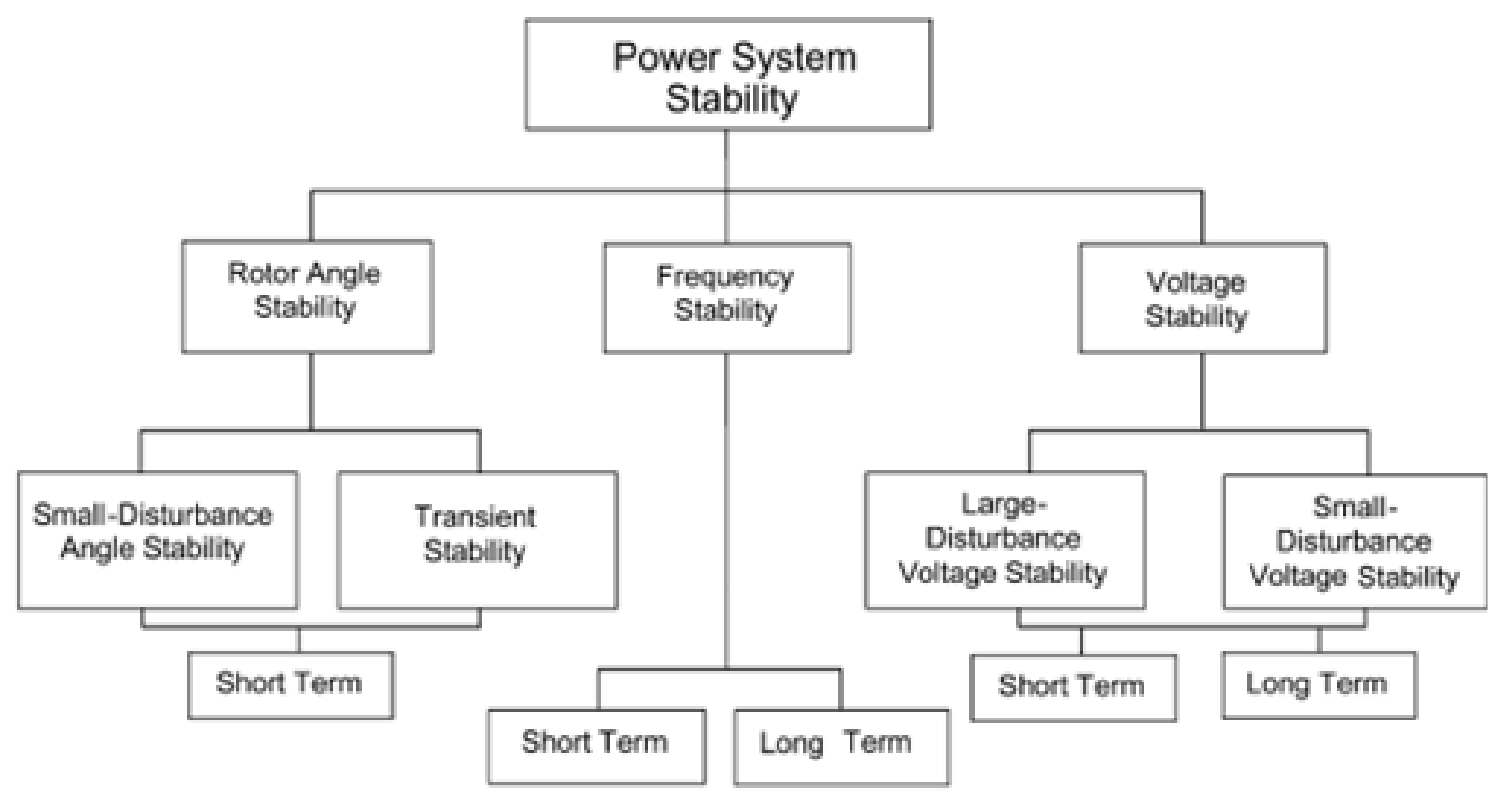

Figure 2.1 Power System Stability Classifications [8]

\subsubsection{Rotor Angle stability}

The rotor angle stability is defined as the ability of interconnected synchronous

machines to remain in synchronism under normal operating conditions and after being subjected to a disturbance. In general, the total active electrical power fed by the generators must always be equal to the active power consumed by the loads; this includes also the losses in the system. This balance between the load and generation can be associated to the balance between the generator input, or mechanical torque, and the generator output, or electrical torque. A disturbance to the system can upset this equilibrium, which results in the acceleration or deceleration of the rotors of the generators. If one generator temporarily runs faster than another, the angular position of its rotor relative to that of the slower machine will increase. The resulting angular difference transfers a part of the load from the slow machine to the fast machine, depending on the theoretically known power angle relationship. This tends to reduce the 
speed difference and hence the angular separation. A further increase in angular separation results in a decrease in power transfer, which can lead to further instability.

\subsubsection{Frequency stability}

Frequency stability refers to the ability of a power system to maintain steady frequency following a severe system upset, resulting in a significant imbalance between generation and load. It depends on the ability to maintain or restore equilibrium between system generation and load, with minimum unintentional loss of load. Instability that may result occurs in the form of sustained frequency swings. A typical cause for frequency instability is the loss of generation causing the overall system frequency to drop. Generally, frequency stability problems are associated with inadequacies in equipment responses, poor coordination of control and protection equipment, or insufficient generation reserve.

\subsubsection{Voltage Stability}

The third power system stability problem is the voltage stability and is elaborated in section 2.2. Further analyses and the method proposed in the framework of this thesis are focused only on this last type of power system stability.

\subsection{Voltage Stability}

Voltage stability is a subset of overall power system stability. It refers to the ability of a power system to maintain steady voltages at all buses in the system after being subjected to a disturbance from a given initial operating condition.

The voltage stability definitions according to $[8]$ are as follows: 
A power system at a given operating point is small-disturbance voltage stable if, after a small disturbance, voltages near loads are similar or identical to pre-disturbance values.

A power system at a given operating point and subject to a given disturbance is voltage stable if voltages near loads approach post disturbance equilibrium values.

A power system at a given operating point and subject to a given disturbance undergoes voltage collapse if post-disturbance equilibrium voltages are below acceptable limits. Voltage collapse may be system wide or partial.

The main factor contributing to voltage instability is usually the voltage drop that occurs when real and reactive power flow through inductive reactance's associated with the transmission network; as the line currents during various power flow conditions increase, the reactive losses increase. When reactive losses increase, the voltage magnitude decreases. Therefore, as the real power flow increases the voltage magnitudes tend to decrease. There is a point that the system can no longer support the real power flow on the lines and maintain a stable voltage. Thusly the voltage collapses.

\subsubsection{Classifications}

As previously mentioned, voltage stability can be classified according to the type of disturbance or to time frame. Small disturbance voltage stability is concerned with a system's ability to control voltages following small perturbations such as incremental changes in system load. This form of stability is determined by the characteristics of loads, continuous controls, and discrete controls at a given instant of time. 
Large disturbance voltage stability is concerned with a system's ability to control voltages following large disturbances such as system faults, loss of generation, or circuit contingencies. This ability is determined by the system-load characteristics and the interactions of both continuous and discrete controls and protections.

In short-term (transient) voltage stability the time frame ranges from zero to ten seconds and involves dynamics of fast acting load components such as induction motors, electronically controlled loads, and HVDC converters. Long term transients on the other hand, correspond to stabilizing elements. This includes load tap changers, voltage regulators, and compensators and their responses to the system changes and the new system conditions. While stabilizing the system, if these elements exceed their operating limits, they will be removed from system operation. This will then drive the system to more severe operating conditions that are uncontrollable. This particular time period may extend to several or many minutes.

In summary, in any given situation, each form of stability are closely associated with one another, and thus one form of instability can possibly lead to another form. Distinguishing between these different forms can provide convenience in identifying the underlying causes of instability, applying an appropriate analysis and ultimately, taking corrective measures to return the system to a stable operating point. With this in mind, the following section will provide an overview on the existing methods used to analyze voltage stability.

\subsection{Voltage Stability Limitations}

Various system aspects may cause voltage instability. Amongst the most important aspects are generators, transmission lines, and loads. [9] 
Generators play an important role for providing adequate reactive power support for power systems. Reactive power is produced by generators and therefore limited by the current rating of the field and armature windings. Since there are levels to how much current or heat the exciter winding can take before the winding becomes damaged, the maximum reactive power output is set using an over excitation limiter (OXL). When the OXL hits the limit, the terminal voltage is no longer maintained constant. Therefore the power transfer limit is further limited, resulting in long-term voltage instability.

Transmission networks are other important constraints for voltage stability. Under a deregulation environment where bulk power is transferred across long distances, the maximum deliverable power is limited by the transmission system characteristics. Power beyond the transmission capacity determined by thermal or stability considerations cannot be delivered.

The third major factor that influences voltage instability is system loads. Voltage instability is load driven. Following a change in the demand, the load will at first change according to its instantaneous characteristic such as, constant impedance or current. It will then adjust the current drawn from the system until the load supplied by the system satisfies the demand at the final system voltage. Similarly when there is a sudden change in system voltage, such as, following a disturbance, the load will change momentarily. It will then adjust the current and draw from the system, whatever current is necessary in order to satisfy the demand. [10]

Another important load aspect is the Load Tap Changing (LTC) transformer, which is one of the key mechanisms in load restoration. In response to a disturbance, the LTC tends to maintain constant voltage level at the low voltage end. Therefore, load 
behavior observed at high voltage level is close to constant power, which may aggravate voltage instability problems.

\subsubsection{Voltage Collapse Incidents}

Many voltage instability incidents have occurred around the world. Table 2-1

groups several known incidents and their time frame.

Table 2-1 Voltage Collapse Incidents [8]

\begin{tabular}{|c|c|c|}
\hline Date & Location & Time Frame \\
\hline 1-Dec-87 & Western France & $4-6$ minutes \\
\hline 22-Aug-87 & Western Tennessee & 10 seconds \\
\hline 23-Jul-87 & Tokyo, Japan & 20 minutes \\
\hline 30-Nov-86 & SE Brazil, Paraguay & 2 seconds \\
\hline 27-Dec-83 & Sweden & 55 seconds \\
\hline 30-Dec-82 & Florida & $1-3$ minutes \\
\hline 4-Aug-82 & Belgium & 4.5 minutes \\
\hline 19-Dec-78 & France & 26 minutes \\
\hline 22-Aug-70 & Japan & 30 minutes \\
\hline
\end{tabular}

From table 2-1 voltage collapse incidents dynamics span can range from seconds to as long as tens of minutes. Since the voltage instability issue started to emerge, research efforts from the power engineering community have been dedicated to studying the voltage instability mechanism and to developing analysis tools and control schemes to mitigate the instability. 


\subsection{Voltage Stability Analysis}

In large complex meshed networks, power-flow analysis is commonly used. In this section an introduction to power-flow, or load flow, analysis and its application to voltage stability will be given in order to understand the voltage stability indices exposed in the next chapter.

\subsubsection{Power-Flow Analysis [11]}

For load flow studies, a balanced three-phase steady-state system is assumed, and thus positive sequence networks are only used. There are four variables associated with each bus in a system: voltage magnitude, phase angle, net real power, and reactive power supplied to each bus. At each bus two of these variables are specified. Each bus is further categorized into one of the following bus types:

1. Swing bus - There is only one swing bus which serves as a reference bus for which $V \angle \delta$, typically $1.0 \angle 0^{\circ}$ per unit, is the input data.

2. Load bus $-\mathrm{P}$ and $\mathrm{Q}$ are the input data. $\mathrm{V}$ and $\delta$ are computed

3. Voltage controlled bus $-\mathrm{P}$ and $\mathrm{V}$ are input data. $\mathrm{Q}$ and $\delta$ are computed.

The network equations in terms of the bus admittance matrix can be written as follows:

$$
\left[\begin{array}{c}
\bar{I}_{1} \\
\bar{I}_{z} \\
\vdots \\
\vdots \\
\bar{I}_{n}
\end{array}\right]=\left[\begin{array}{ccccc}
Y_{11} & -Y_{1 z} & -Y_{1 \mathrm{z}} & \cdots & -Y_{1 n} \\
-Y_{1 z} & Y_{2 z} & -Y_{23} & \cdots & -Y_{n n} \\
\vdots & \vdots & \vdots & \ddots & \vdots \\
-Y_{n 1} & -Y_{n z} & -Y_{n z} & \cdots & Y_{n n}
\end{array}\right]\left[\begin{array}{c}
\bar{V}_{1} \\
\bar{V}_{z} \\
\vdots \\
\vdots \\
\bar{V}_{n}
\end{array}\right]
$$

Where

$\mathrm{n}$ is the total number of buses.

$Y_{i i}$ is the self admittance at node $\mathrm{i}$. 
$Y_{i j}$ is the mutual admittance between buses.

$\bar{V}_{i}$ is the phasor voltage to ground at bus $\mathrm{i}$.

$\bar{I}_{i}$ is the phasor current at bus $\mathrm{i}$.

Equation (2.1) would be linear if injections $\bar{I}_{i}$ were known, but, in practice, are not known for most buses. The current at any node $k$ is related to $P, Q$ and $V$ as follows:

$$
\bar{Y}=\frac{P_{k}+j Q_{k}}{\bar{V}_{k}^{*}}
$$

The relations between $P, Q, V$ and $I$ are defined by the characteristics of the devices connected to the buses, this dependency makes the problem nonlinear and therefore have to be solved iteratively using techniques such as Gauss-Seidel or Newton-Raphson. Due to the complexity in calculations, in practice load flow programs such as ETAP, PSS/E or GE PSLF, PowerWorld is used. In this thesis, all load flow solutions will be obtained using PowerWorld Simulator.

\subsubsection{PV and QV Curves [8]}

Before describing more complex analysis methods, a simple example is given using PV and QV curves, which are two power flow methods widely used to visualize and determine the voltage stability phenomenon.

Consider the simplified two-bus model in Figure 2.2. A generator with magnitude $\mathrm{V}_{1}$, behind a purely reactive line, supplies a constant load with real $\mathrm{P}$ and reactive $\mathrm{Q}$ components. 


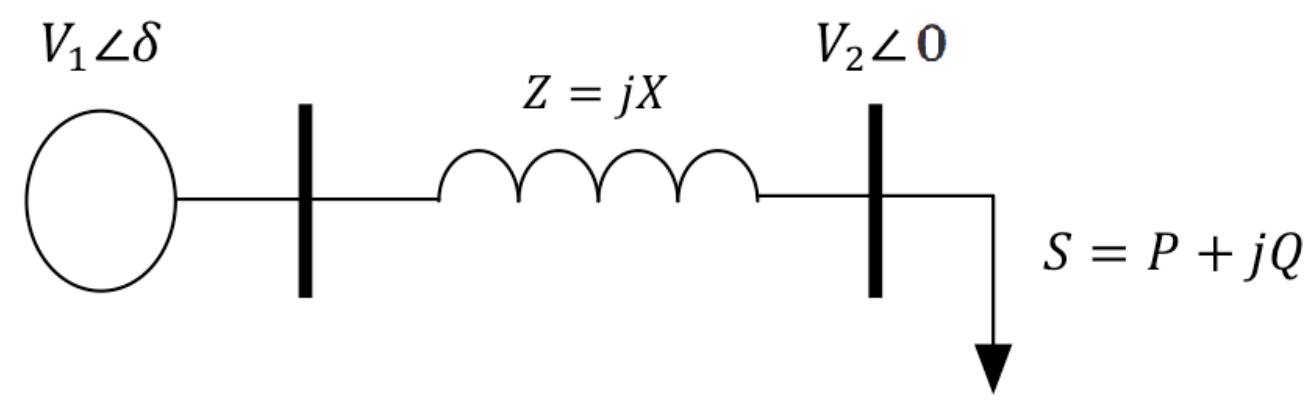

Figure 2.2 Two Bus System

Where $\mathrm{V}$ is voltage at the load bus and $\delta$ is the phase difference between the load and generator and $\theta$ is the line impedance angle. The power delivered to the load is given by equations:

$$
P=\frac{V_{1} V_{2}}{X} \sin \delta
$$

$$
Q=\frac{V_{1} V_{2}}{X}\left[\cos (m i)-\frac{V_{2}^{2}}{X}\right.
$$

Using equations (2.3) and (2.4) to eliminate $\delta$, the following equation is obtained

$$
V_{z}=\sqrt{\frac{V_{1}^{2}}{2}-Q_{L} X \pm X \sqrt{\frac{V_{1}^{2}}{4 X^{2}}-P_{L}^{2}-Q \frac{V_{1}^{2}}{X}}}
$$

As shown in Equation 2.5, the load voltage $V_{z}$ depends on the sending end voltage $V_{1}$, line impedance $\mathrm{X}$, and load demand values, $\mathrm{P}$ and $\mathrm{Q}$ 
The solutions of (2.5) with respect to load voltage $\mathrm{V}$ for different power factors $(\mathrm{Q}=\mathrm{P}(\tan \varphi))$ are shown in figure 2.3

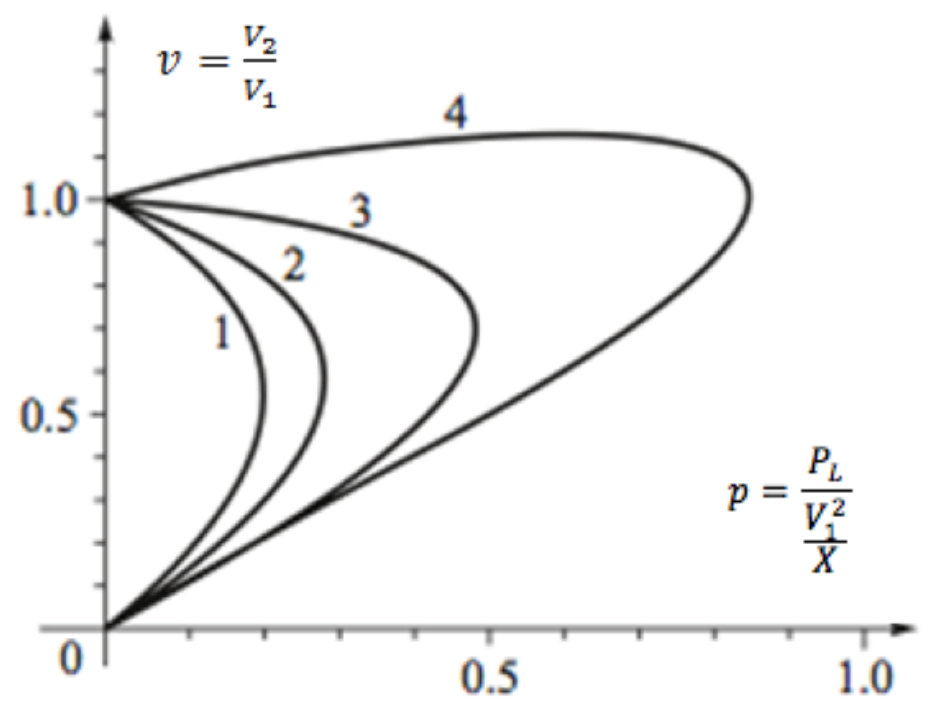

Figure 2.3 PV curves for corresponding power factors: (1) $\varphi=45^{\circ}$ lagging, (2) $\varphi=30^{\circ}$ lagging, (3) $\varphi=0$, (4) $\varphi=30^{\circ}$ leading

For each PV curve there are two equilibrium points, one at high voltage and therefore low current, and the other at low voltage at high current. In practice the high voltage equilibrium is the more stable and the equilibrium at which a power system operates. The tip of the curve represents that maximum power delivered. At leading power factors the maximum power is higher. Using this method, changes in voltage with respect to power can be observed. The plots can serve as a visual on how far the bus is from instability [12]; however there are several disadvantages of this method. Typically power flow can only compute the upper part of the curve up to maximum loading point, beyond this point, the load flow will diverge because it cannot find a solution.

Now, considering the same circuit in figure 2.2, QV curves can be plotted for the load bus. QV curves represent a plot of reactive power versus voltage for a particular bus. 
In order to create a $\mathrm{QV}$ curve, a constant real power load is assumed and a fictitious synchronous condenser with infinite reactive capability is placed on the test bus. The voltage of the synchronous condenser is then varied, and the VAR output is allowed to be any value to meet the scheduled voltage. Reactive power demand is plotted for various voltage schedules as shown in figure 2.4 .

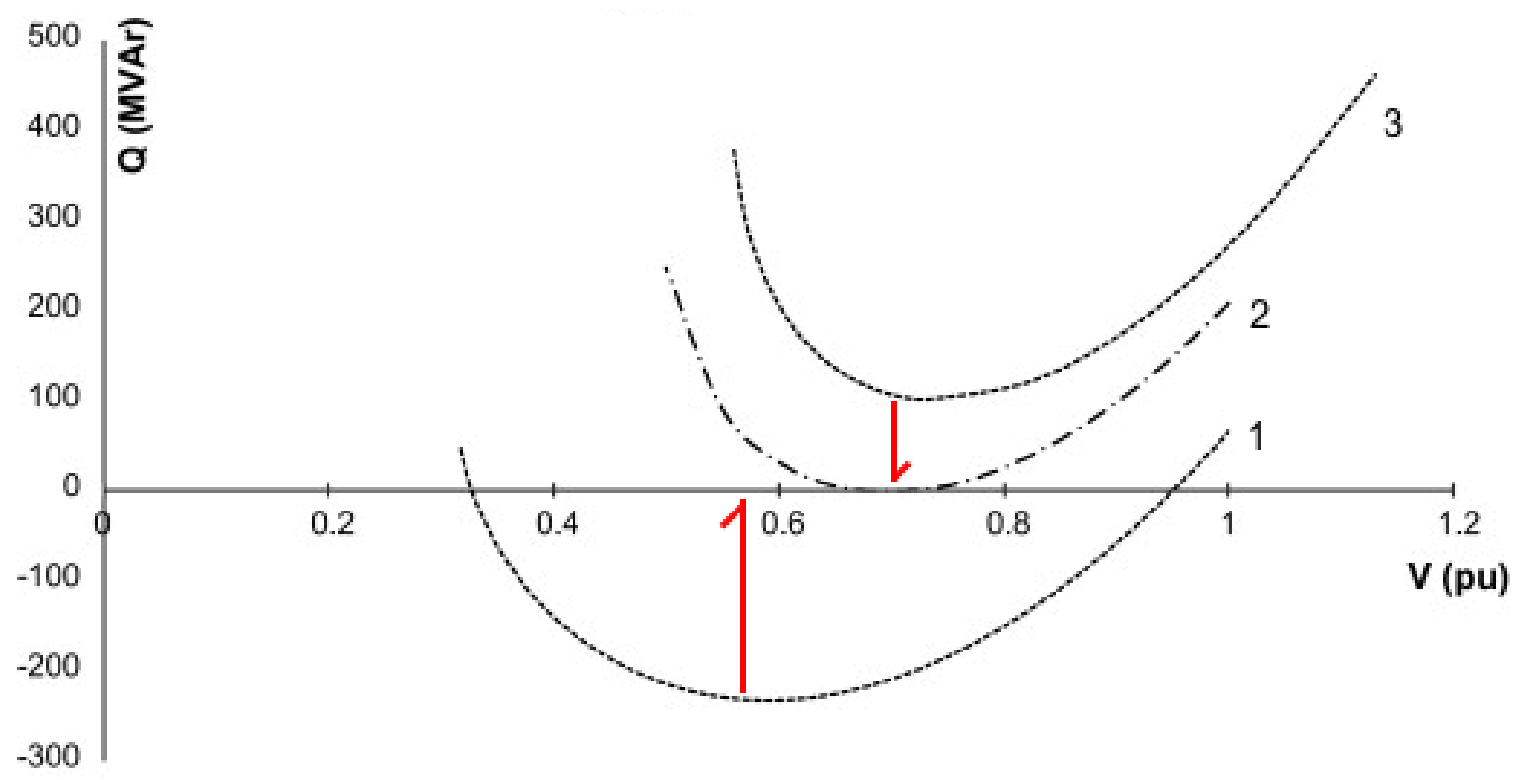

Figure 2.4 QV curves for various load levels. [13]

From figure 2.4, the $\mathrm{x}$-intercept of the curve on the positive slope represents the base case operating point. Tracing downward along the curve represents an increase in MVAR load along with a corresponding decrease in voltage. The positive slope of the QV curve, where $\mathrm{dQ} / \mathrm{dV}>0$, is the stable region and the negative slope of the $\mathrm{QV}$ curve where $\mathrm{dQ} / \mathrm{dV}<0$, is the unstable region. The bottom of the curve where $\mathrm{dQ} / \mathrm{dV}=0$ shows the minimum reactive power required for stable operation, as well as the minimum voltage that the bus can withstand. Curve 1 shows a positive reactive margin, and thus is more robust in terms of voltage stability. Curve 2 has little or no margin, and is therefore 
marginally stable. Any further increase in demand will result in voltage collapse. Curve 3, has no $\mathrm{x}$-intercept, has a negative margin and therefore the system has collapsed. As a result the system will require additional MVAR support in order to come out of the collapse.

This method is useful as it provides the reactive power margin with respect to reactive power injections at the test bus. In conjunction to PV-curves, these two methods are useful for studying voltage stability for small networks, especially radial systems. However, it is not efficient for large meshed systems since it requires extensive computation and may contain more than one critical bus to be analyzed.

\subsubsection{Voltage}

Voltage is probably the most intuitive index for quantifying voltage stability. Monitoring voltage magnitudes have been a widely accepted index to initialize remedial actions such as undervoltage load shedding schemes in order to prevent voltage collapse. Typically, a voltage margin is set between $85 \%-90 \%$ of the nominal voltage. At this point a designated relay will trip, or dispatchers can act accordingly to drop a load in order to prevent voltage collapse.

The limitation of the voltage-based index is that it cannot quantify the distance to the voltage marginally stable point. The bus with the lowest voltage is not necessarily the one closest to the voltage collapse point. As shown in figure 2.3 - curve 4, a load bus with high reactive power compensation may not show any significant low voltage problems even if the power transfer is close to the system transmission limit and the system is close to the voltage marginally stable point. 


\subsubsection{Analysis Methods}

Methods for analysis voltage stability can by classified into two separate categories: Dynamic analysis or Static analysis. Each method is specific in addressing particular problems and has its advantages and disadvantages.

Dynamic analysis can provide information on system performance with respect to time usually ranging from seconds to several minutes. Using dynamic simulations, analyses can be conducted on coordination of equipment where time frames are overlapping; e.g. generator controls, switched capacitor banks and undervoltage load shedding. Dynamic simulations have also proven useful for presenting the system performance during the final stages of collapse such as motor stalling, changes in load, and recovery resulting in voltage collapse or stability respectively. This extra dimension of time adds another layer of complexity in the representation of the system. Each piece of equipment requires detailed set of parameters and equations, and for large systems requires extensive computing power in order to cope with the nonlinearities and switching events.

On the other hand static analyses are often adequate in addressing the slower forms of voltage stability. Indices can be also generated which define areas prone to voltage instability and indicate elements, which are important in the instability phenomenon. These methods are often computationally less intensive, which makes it suitable for online, and offline applications. 


\subsection{Synchrophasors}

As the improvement of the transmission network becomes more complex, the need for faster clearing times, pilot protection schemes and more wide-area protection and control systems to ensure that these lines are being most utilized, has increased. Protection and control systems must adapt to constant changes in the network. The variability on both the supply and demand side increases the importance of having widearea protection, control and monitoring systems that are secure, reliable, and simple as possible. At the heart of these new wide-area systems are time synchronized phasor data known as synchrophasors. Phasor representation of sinusoidal signals is commonly used in ac power system analysis. The sinusoidal waveform defined in equation (2.5):

$$
x(t)=X_{m} \cos (\omega t+\varphi)
$$

The synchrophasor representation $X$ of a signal $\mathrm{x}(\mathrm{t})$ is the complex value given by:

$$
X=\left(\frac{X_{m}}{\sqrt{2}}\right) e^{j \varphi}=\left(\frac{X_{m}}{\sqrt{2}}\right) \mathbb{I}\left(\cos [\varphi+j \sin \varphi)=X_{\eta}+j X_{\mathrm{i}}\right.
$$

Where the magnitude is the root-mean-square (rms) value, $\mathrm{Xm} / \sqrt{ } 2$, of the waveform, and the subscripts $r$ and i signify real and imaginary parts of a complex value in rectangular components. 


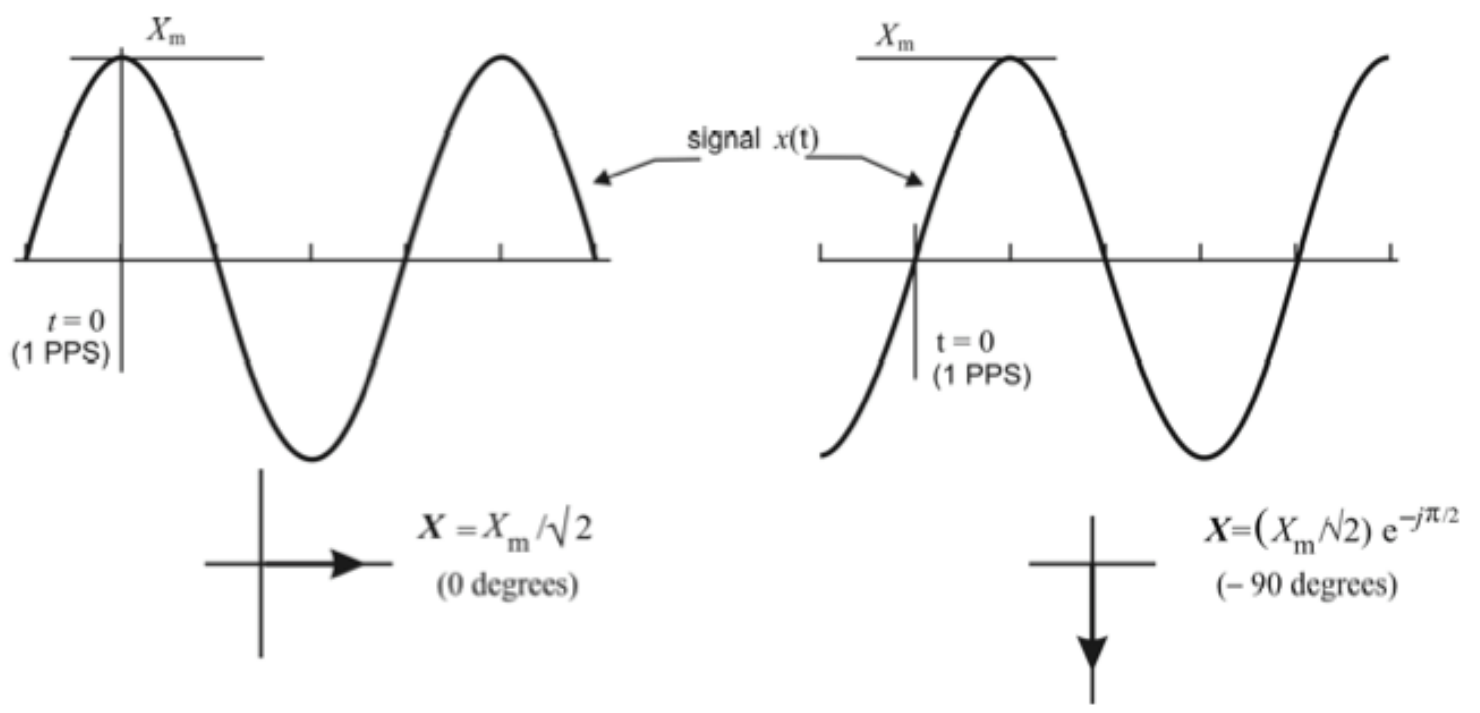

Figure 2.5 Convention for Synchrophasor Representation [14]

In power systems it is common to represent $X$ in phasor notation: $X=X_{m}<\varphi$. The phase angle $\varphi$ depends on the time scale, particularly where $t=0$. It is important to note this phasor is defined for the angular frequency $\omega$ given by $2 \pi f$ where $f$ is the frequency. The representation of a synchrophasor is shown in figure 2.5. IEEE Standard C37.118.1 defines synchrophasors as precise time-synchronized measurements of certain parameters on the electricity grid from phasor measurement units (PMUs). PMUs measure voltage, current and frequency and calculate phasors, and this group of time synchronized grid condition data is called phasor data. Each phasor measurement is time stamped against Global Positioning System universal time. This allows measurements taken by PMUs in different locations or by different owners, to be transmitted over standard communication systems including Ethernet, phone modem, or just an EIA-232 cable from the PMU, then to be synchronized to a computer at each end of the line [15]. 
Now that these values are available from devices on the power system, the question is how to make it available to use this value. One example is using the synchrophasors to define an accurate system model [15]. System models are useful to a system operators and operations engineers as they can provide insight to how power is directed when the network topology is subject to changes. Changes that maybe beneficial to an economic standpoint, such as importing bulk power across line, can be undesirable from a stability standpoint, such as loading the line to its maximum ratings. By having an accurate model of the system, both security and economic factors can be optimized. Consider an equivalent pi model of a transmission line of a two-bus system. When voltage and current measurements are taken at the buses, the line parameters are given by:

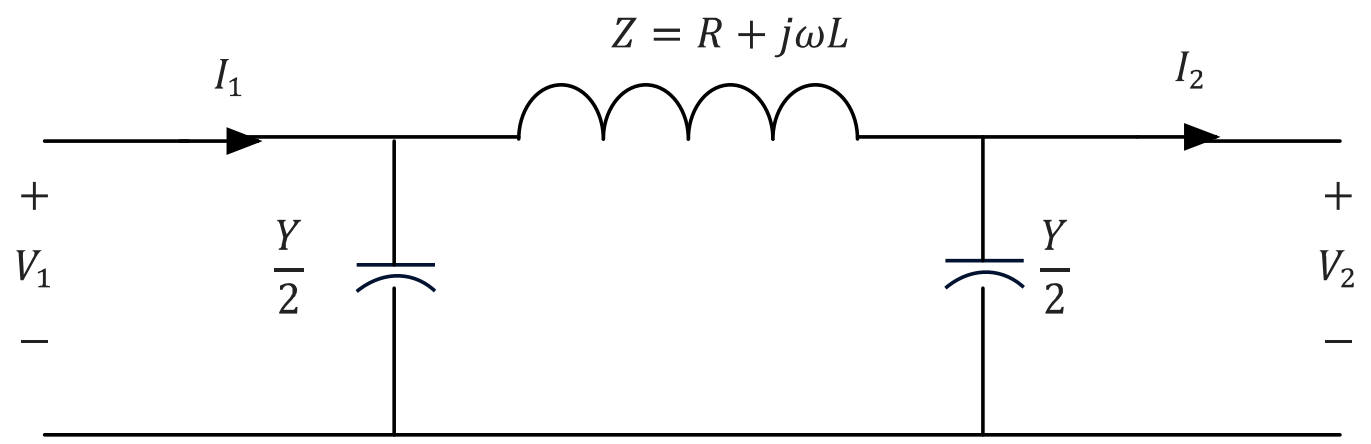

Figure 2.6 Two-bus Pi-Model Equivalent

$$
\begin{aligned}
& I_{1}=\left(V_{1}-\frac{Y}{2}\right)+\left(\frac{V_{1}-V_{2}}{Z}\right) \\
& I_{2}=\left(\frac{V_{1}-V_{2}}{Z}\right)-\left(V_{2} \cdot \frac{Y}{2}\right)
\end{aligned}
$$

Where 


$$
Z=R+j \omega L
$$

Solving for $\mathrm{Y}$

$$
Y=2 \cdot\left(\frac{I_{1}-I_{2}}{V_{1}+V_{2}}\right)
$$

The line parameters are given by

$$
Z=\left(\frac{V_{1}^{2}-V_{2}^{2}}{I_{1} V_{2}-I_{2} V_{1}}\right)
$$

$$
\begin{gathered}
L=\frac{\operatorname{Im}(Z)}{2 \pi f} \\
C=\frac{\operatorname{Im}(Y)}{2 \pi f}
\end{gathered}
$$

By itself these equations can provide an approximate model of a transmission line; however when synchronized to time, the model over periods of time can be useful in many ways. New devices such as the synchrophasor vector processor, have been developed to detect system fluctuations, calculate voltages on far ends of lines, and serve as back up line protection, all at relay speeds. Modern relays can even provide synchrophasor measurements to detect abnormal systems conditions without any additional processors or computers. With a more direct and frequent measurement of the state, the use of synchrophasor technology makes it desirable to further implement into power systems to provide a more robust protection, control and operation performance. 
For the most part, this thesis will focus on using the steady state measurements, and individual snapshots in time, to generate an analysis. Since actual synchrophasor data are continuous measurements, a continuous analysis can be conducted using a simple microprocessor to compute and process the information on a real time basis. 


\section{CHAPTER 3 EVALUATION OF VOLTAGE STABILITY INDICES}

In voltage stability studies, it is crucial to have an accurate knowledge of how close a power system's operating point is from the voltage stability limit. It has been observed that voltage magnitudes alone, do not give a good indication of proximity to voltage stability limit. Therefore, it is useful to assess voltage stability of a power system by means of a voltage stability index (VSI), a scalar magnitude that can be monitored as system parameters change. These indices should be capable of providing reliable information about the proximity of voltage instability in a power system and should reflect the robustness of a system to withstand outages or load increase. Likewise, these indices should be computationally efficient and easy to understand. Therefore operators can use these indices to know how close the system is to voltage collapse in an intuitive manner and react accordingly. This section will provide an overview on various indices proposed in literature. Their characteristics and classifications will be reviewed in their application to analyze voltage stability.

\subsection{Index from Load Flow Jacobian}

\subsubsection{Modal Analysis of Power Flow Model}

Gao, Morison and Kundur [16] introduce an index based on the Jacobian matrix using modal analysis techniques. The Jacobian matrix equation (3.1) is obtained through load flow solutions for a system operating under stable conditions. Given the fact that the system voltage is affected by both changes in real and reactive power, the Jacobian is 
useful as it can provide information on voltage stability.

$$
\begin{gathered}
I=\left[\begin{array}{ll}
\frac{\partial P}{\partial \theta} & \frac{\partial P}{\partial V} \\
\frac{\partial Q}{\partial \theta} & \frac{\partial Q}{\partial V}
\end{array}\right] \\
{\left[\begin{array}{l}
\Delta P \\
\Delta Q
\end{array}\right]=\left[\begin{array}{ll}
J_{11} & J_{12} \\
/ 21 & I_{22}
\end{array}\right]\left[\begin{array}{l}
\Delta \theta \\
\Delta V
\end{array}\right]}
\end{gathered}
$$

By letting $\Delta P=0$, a linearized relationship between the incremental changes in bus voltage and bus reactive power injection can be represented by equation (3.3)

$$
\Delta Q=I_{R} \Delta V
$$

Where $I_{R}$ is the reduced jacobian and given by

$$
I_{R}=\left[J_{2 \mathrm{z}}-J_{21} J_{11}^{-2} J_{1 \mathrm{z}}\right]
$$

The modes of the system can be defined by the eigenvalues and eignvectors of $J_{\mathbb{R}}$ Assume

$$
I_{R}=\xi M
$$

Where $\xi$ is the right eigenvector matrix of $I_{R}: \Lambda$ is the diagonal eigenvalue matrix of $I_{R} ; \eta$ is the left eigenvector matrix of $I_{R}$. Then

$$
I_{R}^{-1}=\xi \Lambda^{-1} \eta
$$

Incremental changes in reactive power and voltage are related by equation 3.3.

Substituting equation 3.5 , then 


$$
\Delta V=\xi \Lambda^{-1} \eta \Delta Q
$$

Or

$$
\Delta V=\sum_{i} \frac{\xi_{i} \eta_{i}}{\lambda_{i}} \Delta Q
$$

Where $\lambda_{i}$ is the ith eigenvalue, $\xi_{z}$ is the ith column right eigenvector, and $\eta_{i}$ is the ith row left eigenvector. Therefore the ith modal reactive power variation is

$$
\Delta Q_{m i}=k_{i} \xi_{i}
$$

Where $k_{i}$ is a normalization factor such that

$$
k_{i}^{2} \sum_{i} \xi_{j i}^{2}=1
$$

The ith modal variation can be written

$$
\Delta V_{m i}=\frac{1}{\lambda_{i}} \Delta Q_{m i}
$$

The voltage stability index can then be defined by the mode of eigenvalue $\lambda_{2}$. The minimum eigenvalue in a power system is the global VSI value. A Larger value of $\lambda_{i}$ will give smaller changes in the voltages when a small disturbance occurs. The system is stable when the eigenvalue of $I_{R}$ is positive. When the eigenvalue reaches zero, the system is marginally stable. If one of the eigenvalues becomes negative the system is unstable.

Left and right eigenvectors corresponding to the critical modes in the system can 
provide information concerning the mechanism of voltage instability. From this, the bus participation matrix is formed. Bus participation factors show the voltage stability of nodes in the power system. The bus participation factor of a bus can be defined as

$$
P_{k i}=\xi_{k i} \eta_{k i}
$$

The row $\mathrm{k}$ of the matrix indicates the bus number and the matrix column $\mathrm{i}$ indicate the system mode. A large bus participation factor indicates how much impact a bus has on a power system.

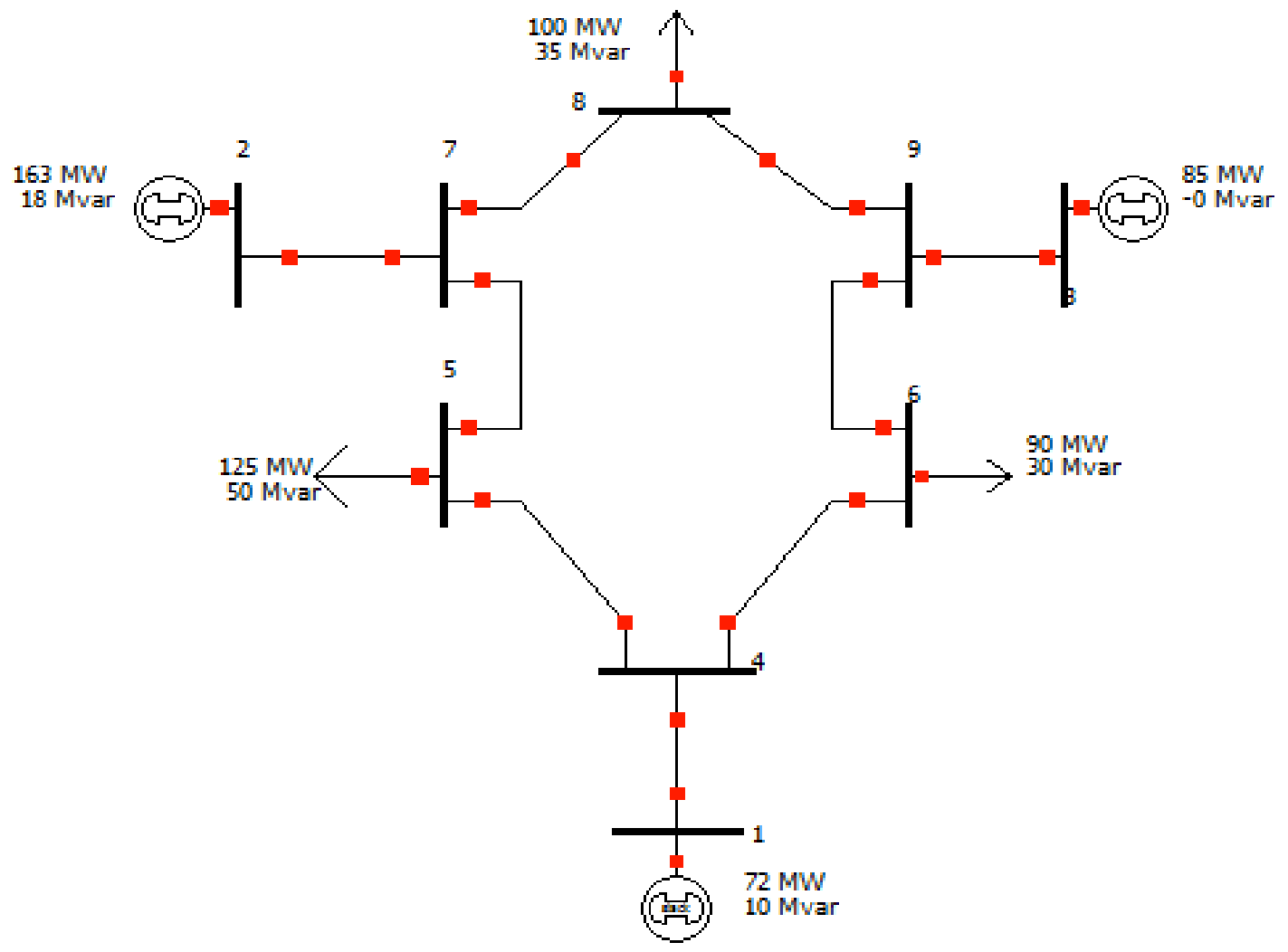

Figure 3.1WSCC 9 Bus System

To demonstrate the modal analysis concept, consider the WSCC system in figure 
3.1. The load flow was run and the Jacobian matrix was obtained to determine the eigenvalues of the system. The system's six non-generating buses resulted in a 6x6Jacobian matrix and six corresponding eigenvalues. Table 3-1 shows the computed eigenvalues after each load increase, The fact that table 3-1 shows no negative values, verifies that the system is stable. The lowest eigenvalue of the initial case is $\lambda_{\mathbf{A}}=6.065$ and therefore the most critical mode. The eigenvalue $\lambda_{4}$ remains the critical mode throughout each load increase. This may not be the case in all situations, as different systems may have different loading patterns and system changes affecting the outcome of the Jacobian matrix. Table 3-1 shows the eigenvalues for a given load increase. The three loads eigenvalues are plotted in figure 3.2.

Table 3-1: Eigenvalues for a given load increase

\begin{tabular}{|c|c|c|c|c|c|c|c|c|}
\hline $\begin{array}{c}\text { Total } \\
\text { MW }\end{array}$ & 350 & 400 & 500 & 550 & 600 & 650 & 700 & 725 \\
\hline$\lambda_{\mathbf{1}}$ & 50.01 & 49.51 & 48.35 & 47.62 & 46.74 & 45.66 & 44.11 & 42.25 \\
\hline$\lambda_{\mathbf{z}}$ & 45.45 & 45.16 & 44.04 & 43.23 & 42.13 & 40.52 & 37.64 & 33.17 \\
\hline$\lambda_{\mathbf{a}}$ & 35.03 & 35.62 & 35.08 & 34.72 & 34.29 & 33.71 & 32.79 & 31.45 \\
\hline$\lambda_{\mathbf{4}}$ & 6.06 & 5.57 & 5.14 & 4.86 & 4.50 & 4.02 & 3.19 & 2.65 \\
\hline$\lambda_{\mathbf{s}}$ & 12.78 & 12.45 & 12.00 & 11.71 & 11.37 & 10.93 & 10.34 & 9.67 \\
\hline$\lambda_{\mathbf{s}}$ & 15.03 & 14.10 & 13.36 & 12.87 & 12.28 & 11.47 & 10.08 & 8.66 \\
\hline
\end{tabular}




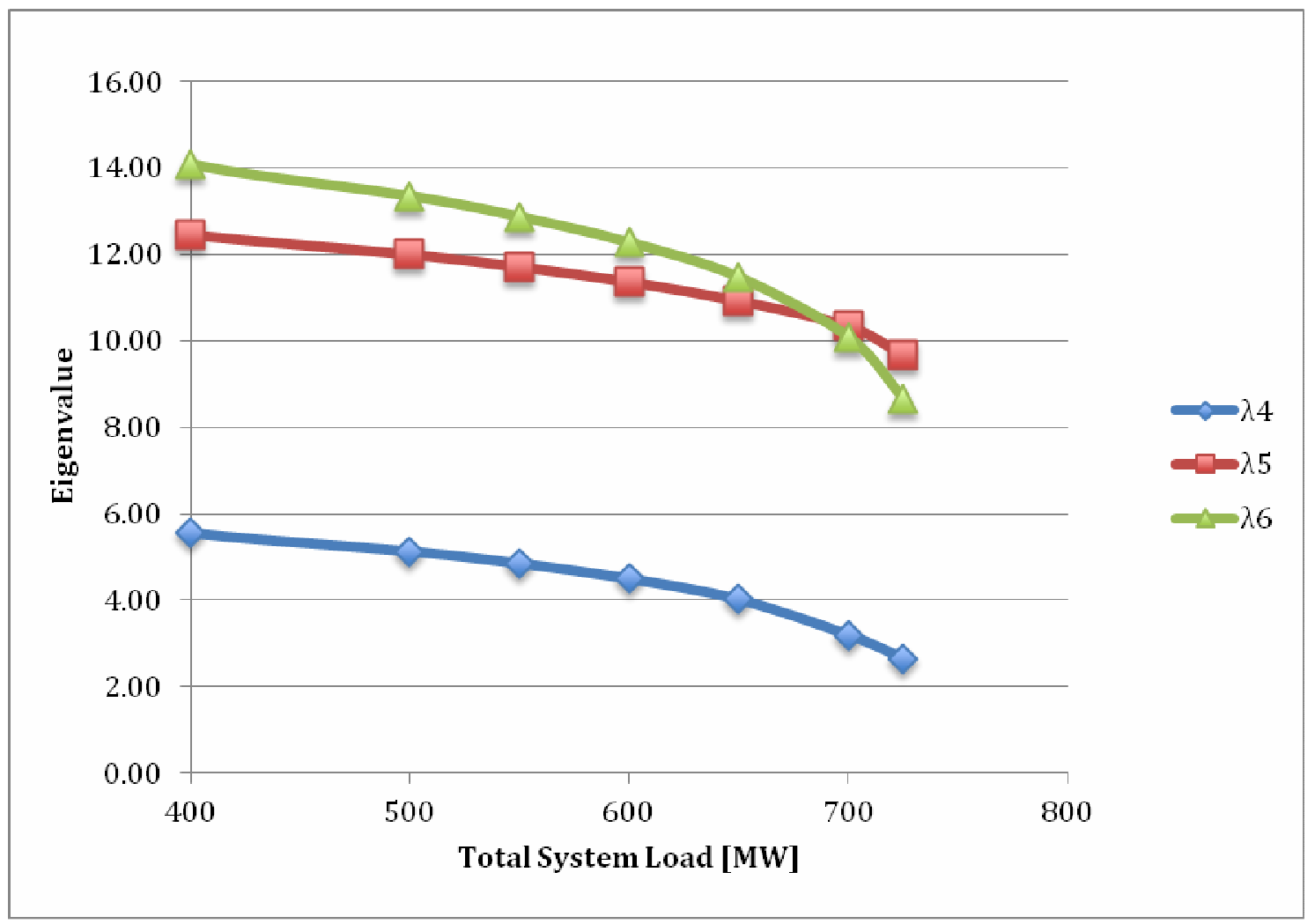

Figure 3.2 Three Lowest Eigenvalues Under System Load Changes.

The participation factor $P$ of each bus for a given modal variation, for the first and last load increases are shown in table 3-2.

Table 3-2 Participation Factors For Each Modal Variation (Initial Conditions)

\begin{tabular}{|c|c|c|c|c|c|c|c|}
\hline & & $\lambda_{i}$ & $\lambda_{2}$ & $\lambda_{g}$ & $\lambda_{4}$ & $\lambda_{\mathbf{s}}$ & $\lambda_{6}$ \\
\hline \multirow{6}{*}{$\stackrel{\mathscr{D}}{\mathscr{n}}$} & 4 & 0.391 & 0.399 & 0.002 & 0.129 & 0.070 & 0.009 \\
\hline & 5 & 0.101 & 0.022 & 0.040 & 0.280 & 0.027 & 0.530 \\
\hline & 6 & 0.063 & 0.034 & 0.052 & 0.289 & 0.223 & 0.338 \\
\hline & 7 & 0.233 & 0.282 & 0.241 & 0.084 & 0.146 & 0.013 \\
\hline & 8 & 0.129 & 0.200 & 0.011 & 0.147 & 0.487 & 0.025 \\
\hline & 9 & 0.082 & 0.062 & 0.654 & 0.070 & 0.046 & 0.085 \\
\hline
\end{tabular}


Table 3-3 Participation Factors For Each Modal Variation (Last Increase Before Collapse)

\begin{tabular}{|c|c|c|c|c|c|c|c|}
\hline & & $\lambda_{1}$ & $\lambda_{2}$ & $\lambda_{3}$ & $\lambda_{4}$ & $\lambda_{3}$ & $\lambda_{s}$ \\
\hline \multirow{6}{*}{ 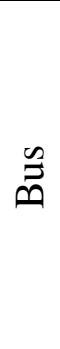 } & 4 & 0.0145 & 0.7055 & 0.0944 & 0.1465 & 0.0357 & 0.0034 \\
\hline & 5 & 0.0176 & 0.0534 & 0.0451 & 0.3996 & 0.1334 & 0.3508 \\
\hline & 6 & 0.0081 & 0.1069 & 0.0027 & 0.3107 & 0.0010 & 0.5707 \\
\hline & 7 & 0.4982 & 0.0768 & 0.2169 & 0.0451 & 0.1215 & 0.0414 \\
\hline & 8 & 0.2866 & 0.0111 & 0.0114 & 0.0663 & 0.5992 & 0.0253 \\
\hline & 9 & 0.1750 & 0.0463 & 0.6295 & 0.0317 & 0.1092 & 0.0084 \\
\hline
\end{tabular}

Results from tables 3-2 and 3-3 reveal that bus $5 \& 6$ are the buses with the highest participation factors under the critical mode, and therefore the bus closest to instability. This method has been demonstrated its effectiveness for voltage stability studies as proven in [17-19]. In sum, eigenvalues can be used as an indicator of the proximity of an operating point to the point of voltage collapse. However this magnitude can vary from system to system, and for different operating points. Offline studies should be done in order to verify a margin to when an eigenvalue determines a collapse.

\subsection{Indices Based on Phasor Measurement Units}

The realization that phasor measurement devices can provide enough information for monitoring voltage stability at local load supply nodes, has promoted research in algorithms that only use voltage and current phasor measurements to monitor system voltage stability [20-25]. Many of the indices presented in this section are based on the assumption that voltage stability is related to the maximum power delivered to a load. This section will describe a method to obtain a Thevenin equivalent parameters followed by a review various indices based on phasor measurements. 


\subsubsection{Algorithm for Equating Thevenin Equivalent Parameters [26-27]}

The buses in a power system can be classified into three categories: load bus, tie bus, and generator bus. A bus with a load attached to it is considered a load bus. A tie bus refers to a bus with no load or any generation device attached. A generator bus includes a bus whose voltage is regulated by an attached generator, as well as a boundary bus, which is modeled by PV bus. A generator bus becomes a load bus if its attached generator reaches its reactive power limit. The injection currents into the system can be written as

$$
I_{s y s}=Y_{s y s} V_{s y s}
$$

Where the Y matrix is known as the system admittance matrix, $\mathrm{V}$ and I stand for the voltage and current vectors. The elements of the admittance matrix with the corresponding voltages can be reorganized into the three types of buses can be expressed as

$$
\left[\begin{array}{l}
i_{L} \\
i_{T} \\
i_{G}
\end{array}\right]=\left[\begin{array}{lll}
Y_{L L} & Y_{L T} & Y_{L G} \\
Y_{T L} & Y_{T T} & Y_{T G} \\
Y_{G L} & Y_{G T} & Y_{G G}
\end{array}\right]\left[\begin{array}{l}
v_{L} \\
v_{T} \\
v_{G}
\end{array}\right]
$$

The subscripts $\mathrm{L}, \mathrm{T}$ and $\mathrm{G}$ represent load bus, tie bus and generator bus, respectively.

The load voltage can be expressed by

$$
V_{L}=V_{\text {open }}-Z_{L L} I_{L}
$$

Where

$$
V_{o p e n}=\sum_{k=1}^{M} H_{L G_{j k}} V_{G_{k}}
$$




$$
\begin{aligned}
& H_{L G}=Z_{L L} \cdot\left(Y_{L T} \cdot Y_{T T}^{-1} \cdot Y_{T G}-Y_{L G}\right) \\
& Z_{L L}=\left(Y_{L L}-Y_{L T} \cdot Y_{T T}^{-1} \cdot Y_{T L}\right)^{-\mathbf{1}}
\end{aligned}
$$

The term $V_{\text {open }}$ represents the open-circuit voltage and $Z_{L L}$ is the self-impedance at the jth load bus. Considering the effect of the other loads, equation (3.19) introduces a coupling voltage term which is related to the mutual-impedance ${ }^{Z_{L L_{i j}}}$ and written as.

$$
V_{L}=Z_{L L_{j j}}\left(\frac{-S_{L_{j}}}{V_{L_{j}}}\right)^{*}+\sum_{k=1}^{M} H_{L G_{j k}} V_{G_{k}}+\sum_{i=1, i \neq j}^{N} Z_{L L_{j i}}\left(\frac{-S_{L_{i}}}{V_{L_{i}}}\right)
$$

The coupling term is then combined with the open-circuit voltage term to form the equivalent voltage as shown in figure 3.3.

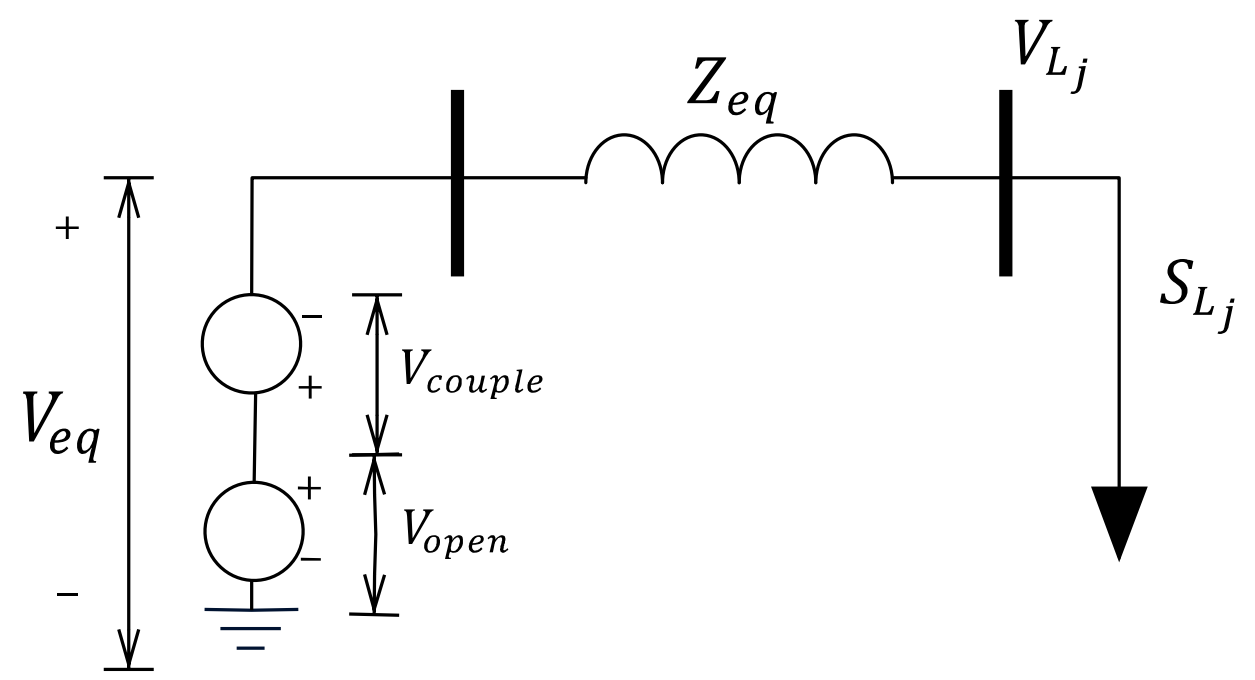

Figure 3.3 Thevenin Equivalent with coupling and open circuit voltage Thus, the Thevenin parameters expressed by 


$$
V_{\theta q_{j}}=V_{\text {open }}-V_{\text {couple }}=\sum_{k=1}^{M} H_{L G_{j k}} V_{G_{k}}-\sum_{i=1, i \neq j}^{N} Z_{L L_{j i}}\left(\frac{S_{L_{i}}}{V_{L_{i}}}\right)^{*}
$$

Where $\mathrm{M}$ is the number of source buses and $\mathrm{N}$ is the number of load buses and

$$
Z_{e q_{j j}}=Z_{L L_{j j}}
$$

Figure 3.3 can then be redrawn as shown on figure 3.4

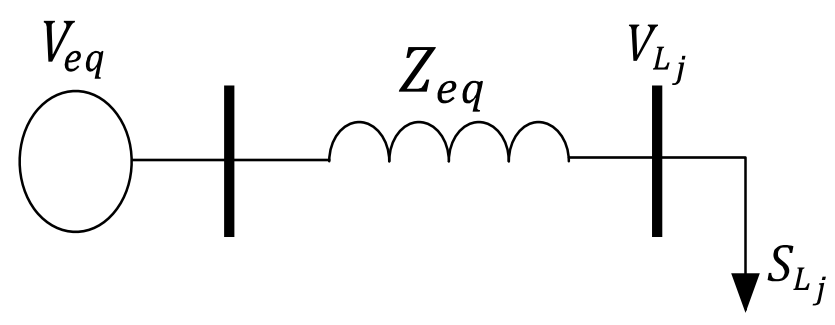

Figure 3.4 Simplified Thevenin Network

From equations (3.20-3.21) the following observations can be made: The equivalent voltage source, $\mathrm{V}_{\mathrm{eq}}$, is a function of the true voltage sources and other system loads and thus Veq decreases as other system loads increase. The equivalent impedance, $Z_{\text {equ }}$, only depends on the system topology and line characteristics. To a power system with a fixed topology, the equivalent impedance remains constant.

\subsubsection{Line Stability Index}

An index based on the power flow concept of a two bus equivalent network has been introduced by Moghavvemi in [28]. The $L_{m n}$ index predicts the stability of a line between two buses and is given by equation (3.22)

$$
L_{m n}=\frac{4 X Q_{r}}{\left[V_{s} \sin (\theta-\delta)\right]^{2}}
$$


Based on the value of the indices of lines, voltage collapse can be predicted. As long as the stability index remains less than 1 , the system is stable. When the index becomes greater than one, the whole system loses its stability and voltage collapse occurs. Therefore the index can be used in voltage collapse prediction.

\subsubsection{Fast Voltage Stability Index}

Similar to the previous index, the Fast Voltage Stability Index (FVSI) is based on the power flow of a two bus equivalent network [29]. The index is calculated as shown in

$$
F V S I=\frac{4 Z Q_{r}}{V_{s}^{2} X}
$$

Values closer to one show the weakest line in the system. This index can also be used to determine the weakest branch in the system. It can also used to determine the weakest bus on the system. The determination of the weakest bus is based on the maximum load allowed on a load bus. The most vulnerable bus in the system corresponds to the bus with the smallest maximum permissible load.

\subsubsection{LQP index}

The LQP index proposed in [30] is based on the concept of power flow through a single line. This index is used to find the stability index for each line connection between two bus bars in an interconnected network.

$$
L Q P=4\left(\frac{X}{V_{g}^{2}}\right)\left(\frac{X}{V_{s}^{2}} P_{s}^{2}+Q_{r}\right)
$$

Values closer to one show the weakest line in the system. 


\subsubsection{VSI based on Maximum Power Transfers}

The Voltage Stability Index (VSI) is based on real, reactive and apparent power across a transmission line [27]. The maximum power transfer across a transmission line is derived from a two bus equivalent single-sourced system. Once the three maximum transferable powers, $P_{\max } \cdot Q_{\max } \cdot S_{\max } \cdot \mathrm{are}$ obtained, the VSI is calculated by the minimum value between the ratio of three load margins

$$
V S I=\min \left(\frac{P_{\text {margin }}}{P_{\max i}}, \frac{Q_{\text {margin }}}{Q_{\max m}}, \frac{S_{\text {margin }}}{S_{\max }}\right)
$$

Where

$$
\begin{aligned}
& P_{\operatorname{margin}}=P_{\max }-P \\
& Q_{\operatorname{margin}}=Q_{\max }-Q \\
& S_{\operatorname{margin}}=S_{\max }-S \\
& P_{\max }=\sqrt{\frac{V_{s}^{4}}{4 X^{2}}-\frac{Q V_{s}^{2}}{X}} \\
& Q_{\max }=\frac{V_{s}^{2}}{4 X^{2}}-\frac{P^{2} X}{V_{s}^{2}} \\
& S_{\max }=\frac{(1-\sin \theta) V_{s}^{2}}{2(\cos \theta)^{2} X}
\end{aligned}
$$

The VSI is then computed which will vary from 0 to 1 . If the index is 1 , the bus is voltage stable and if the index is 0 the voltage at a bus has collapsed. 


\subsubsection{VCPI}

The Voltage Collapse Proximity Index (VCPI) uses the system admittance matrix instead of making a reduced equivalent system [31]. A modified voltage phasor is first computed using the measurement value of voltage phasor at all buses along with the admittance matrix. The VCPI is then computed which will vary from 0 to 1 . If the index is 0 , the bus is voltage stable and if the index is 1 the voltage at a bus has collapsed.

$$
\begin{aligned}
& V C P I_{k t h \text { bus }}=\left|\begin{array}{c}
\sum_{m=1}^{N} V_{m}^{s} \\
1-\frac{V_{k \neq k}}{V_{k}}
\end{array}\right| \\
& V_{m}^{s}=\frac{Y_{k m}}{\sum_{\substack{N=1 \\
j=k}}^{N} Y_{k j}} V_{m}=\left|V_{m}^{s}\right| \angle \delta_{m}^{s}
\end{aligned}
$$

Where $\mathrm{Y}_{\mathrm{km}}$ represents the mutual admittance between $k$ th bus and $m$ th bus.

$\mathrm{Y}_{\mathrm{kk}}$ represents the self-admittance of bus $k$.

$\mathrm{V}_{\mathrm{k}}$ represents the voltage at $k$ th bus.

$\mathrm{V}_{\mathrm{m}}$ represents the voltage at the $m$ th bus

This chapter has presented various voltage stability indices proposed in literature. These indices require few computations and can be suitable for determining the state of the system on an intuitive level. While one index doesn't necessarily perform better than the other, it is useful to understand the strengths and limitations of various indices. 


\section{CHAPTER 4 IMPLEMENTATION OF INDICES AND TEST NETWORKS}

This chapter will first introduce PowerWorld Simulator and the assumptions used for the analysis. A detailed description of two test-networks will be presented. Two indices from chapter 3 will be chosen for analysis. The algorithm used to implement the indices and test cases will be explained.

\subsection{PowerWorld Simulator}

The majority of the works presented are based on simulation done in Powerworld Simulator. PowerWorld is a commercial program used by many utilities in the nation. The program is capable of analyzing a power system in many ways including area transaction economic analysis, power transfer distribution factor (PTDF) computation, short circuit analysis and contingency analysis, and PV/QV analysis. At the core of the software is a robust load flow solution engine. System models can be implemented through its drag and drop visual interface or through a common text input.

For this study, the following load flow assumptions are made: The System frequency is uniform at $60 \mathrm{~Hz}$ with a 100MVA base. All buses contain PMUs, therefore the entire system is observable and can output measurements as described in previous chapters. All generator AVRs regulate at scheduled voltage for all generator bus until the MVAR capability limit has been reached. Loads are constant power loads, unless mentioned otherwise. Transmission lines are based on the equivalent Pi-model containing an equivalent resistance, reactance, and shunt impedance. All lines will assume infinite 
ampacity; however, ratings will be used for analysis purposes only. Lastly, the voltage collapse point is assumed to be when the power flow does not converge to a solution.

\subsection{Test Systems}

This section introduces two test systems to conduct analyses. These systems have been used broadly in literature to verify different aspects of voltage instability. Initially, the power flow is solved to verify the validity of the data. The results are compared with other references available.

\subsubsection{BPA 10-Bus System}

This 10-bus system was modeled after Bonneville Power Administration (BPA) [7]. In this system, three generators feed around $6000 \mathrm{MW}$ of load. One load is a constant power aggregated industrial load and the other is a half constant power, half constant current aggregated residential load. Generators 1 and 2 feed approximate $5000 \mathrm{MW}$ into the load area across five 500-kV transmission lines. The receiving end is heavily VARcompensated by three shunt capacitor banks. 


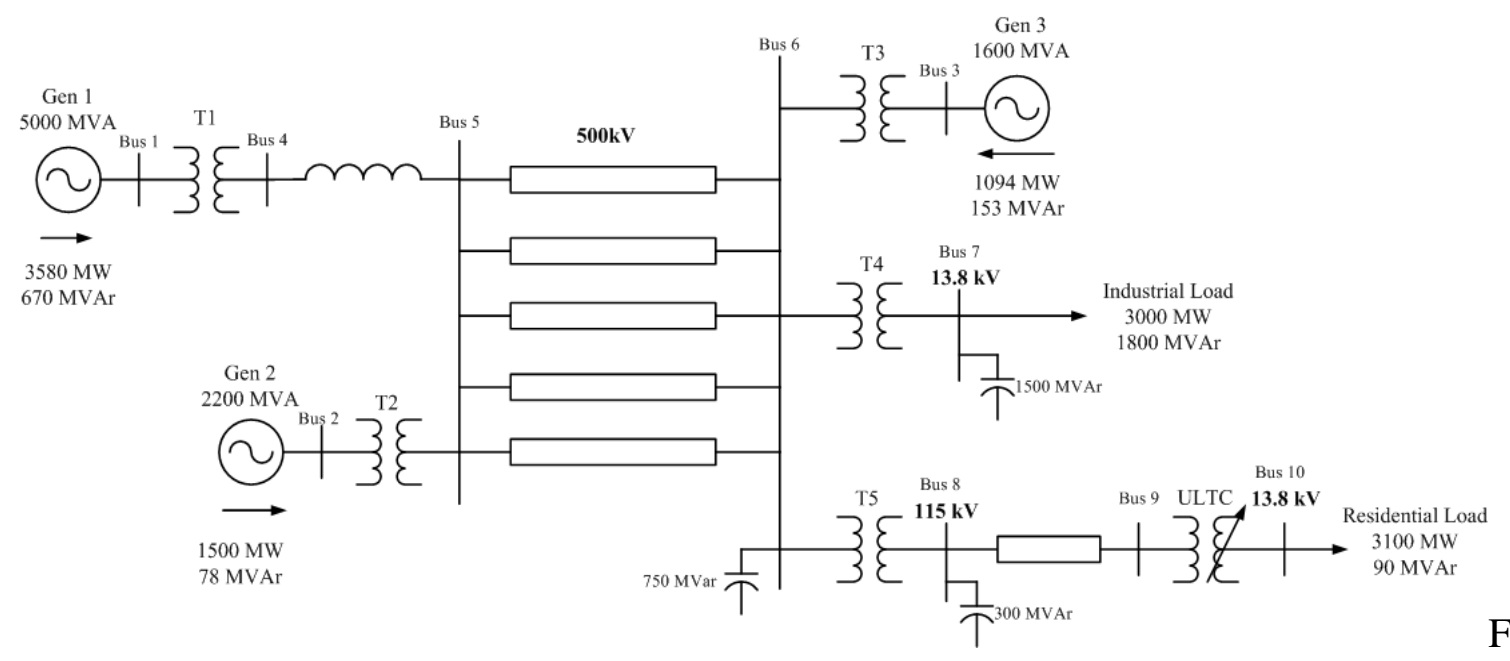

igure 4.1 BPA 10 bus Test System

\subsubsection{IEEE 39-Bus System}

The IEEE 39-Bus power system is based on a $345 \mathrm{kV}$ transmission system in New England Power System as shown on figure 4.2. The system, which is modeled on 100 MVA base, contains 10 machines, 46 lines and 19 Loads. Buses 31 and 39 serve as boundary buses, which is modeled by an aggregated generator and constant power load, contains a reduced network equivalent of the neighboring network. 


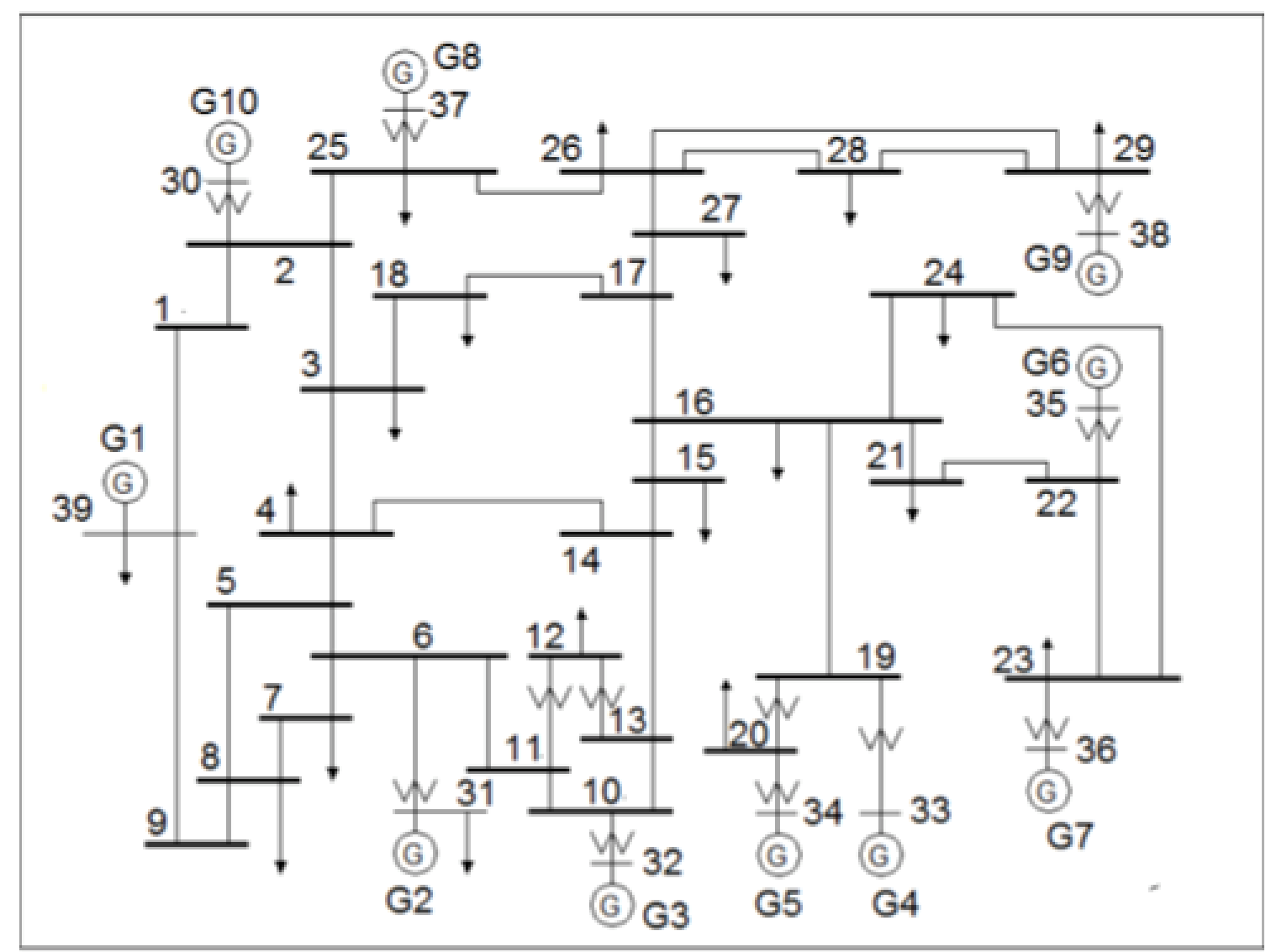

Figure 4.2 IEEE 39-Bus Test System

The system base case conditions are given in per unit values, unless specified otherwise and are shown on tables 4-1,4-2 and 4-3. 
Table 4-1 IEEE 39 Bus Parameters- Load and Generation

\begin{tabular}{|c|c|c|c|c|c|}
\hline BusNum & Bus Types & Voltage [pu] & Load MW & Load MVAR & Gen MW \\
\hline 1 & PV & & & & \\
\hline 2 & PV & & & & \\
\hline 3 & $\mathrm{PV}$ & & 322 & 2.4 & \\
\hline 4 & $\mathrm{PV}$ & & 500 & 184 & \\
\hline 5 & $\mathrm{PV}$ & & & & \\
\hline 6 & $\mathrm{PV}$ & & & & \\
\hline 7 & $\mathrm{PV}$ & & 233.8 & 84 & \\
\hline 8 & PV & & 522 & 176 & \\
\hline 9 & PV & & & & \\
\hline 10 & $\mathrm{PV}$ & & & & \\
\hline 11 & PV & & & & \\
\hline 12 & PV & & 7.5 & 88 & \\
\hline 13 & $\mathrm{PV}$ & & & & \\
\hline 14 & $\mathrm{PV}$ & & & & \\
\hline 15 & $\mathrm{PV}$ & & 320 & 153 & \\
\hline 16 & PV & & 329 & 32.3 & \\
\hline 17 & PV & & & & \\
\hline 18 & $\mathrm{PV}$ & & 158 & 30 & \\
\hline 19 & PV & & & & \\
\hline 20 & $\mathrm{PV}$ & & 628 & 103 & \\
\hline 21 & $\mathrm{PV}$ & & 274 & 115 & \\
\hline 22 & $\mathrm{PV}$ & & & & \\
\hline 23 & $\mathrm{PV}$ & & 247.5 & 84.6 & \\
\hline 24 & PV & & 308.6 & -92 & \\
\hline 25 & $\mathrm{PV}$ & & 224 & 47.2 & \\
\hline 26 & $\mathrm{PV}$ & & 139 & 17 & \\
\hline 27 & $\mathrm{PV}$ & & 281 & 75.5 & \\
\hline 28 & PV & & 206 & 27.6 & \\
\hline 29 & $\mathrm{PV}$ & & 283.5 & 26.9 & \\
\hline 30 & PV & & & & 250 \\
\hline 31 & Swing & 1 & 9.2 & 4.6 & 520.58 \\
\hline 32 & PQ & 0.9831 & & & 650 \\
\hline 33 & PQ & 0.9972 & & & 632 \\
\hline 34 & PQ & 0.9972 & & & 508 \\
\hline 35 & PQ & 1.0493 & & & 650 \\
\hline 36 & PQ & 1.0635 & & & 560 \\
\hline 37 & PQ & 1.0278 & & & 540 \\
\hline 38 & PQ & 1.0265 & & & 830 \\
\hline 39 & PQ & 1.03 & 1104 & 250 & 1000 \\
\hline
\end{tabular}


Table 4-2: IEEE 39 Bus Parameters- Line Data

\begin{tabular}{|c|c|c|c|c|c|}
\hline From Bus & To Bus & LineXfmr & $\mathrm{R}$ & $\mathrm{X}$ & $\mathrm{B}$ \\
\hline 1 & 2 & NO & 0.0035 & 0.0411 & 0.6987 \\
\hline 1 & 39 & NO & 0.001 & 0.025 & 0.75 \\
\hline 2 & 3 & NO & 0.0013 & 0.0151 & 0.2572 \\
\hline 2 & 25 & $\mathrm{NO}$ & 0.007 & 0.0086 & 0.146 \\
\hline 2 & 30 & YES & 0 & 0.0181 & 0 \\
\hline 3 & 4 & NO & 0.0013 & 0.0213 & 0.2214 \\
\hline 3 & 18 & NO & 0.0011 & 0.0133 & 0.2138 \\
\hline 4 & 5 & NO & 0.0008 & 0.0128 & 0.1342 \\
\hline 4 & 14 & NO & 0.0008 & 0.0129 & 0.1382 \\
\hline 5 & 6 & NO & 0.0002 & 0.0026 & 0.0434 \\
\hline 5 & 8 & $\mathrm{NO}$ & 0.0008 & 0.0112 & 0.1476 \\
\hline 6 & 7 & $\mathrm{NO}$ & 0.0006 & 0.0092 & 0.113 \\
\hline 6 & 11 & NO & 0.0007 & 0.0082 & 0.1389 \\
\hline 6 & 31 & YES & 0 & 0.025 & 0 \\
\hline 7 & 8 & $\mathrm{NO}$ & 0.0004 & 0.0046 & 0.078 \\
\hline 8 & 9 & $\mathrm{NO}$ & 0.0023 & 0.0363 & 0.3804 \\
\hline 9 & 39 & $\mathrm{NO}$ & 0.001 & 0.025 & 1.2 \\
\hline 10 & 11 & NO & 0.0004 & 0.0043 & 0.0729 \\
\hline 10 & 13 & NO & 0.0004 & 0.0043 & 0.0729 \\
\hline 10 & 32 & YES & 0 & 0.02 & 0 \\
\hline 12 & 11 & YES & 0.0016 & 0.0435 & 0 \\
\hline 12 & 13 & YES & 0.0016 & 0.0435 & 0 \\
\hline 13 & 14 & NO & 0.0009 & 0.0101 & 0.366 \\
\hline 14 & 15 & $\mathrm{NO}$ & 0.0018 & 0.0217 & 0.366 \\
\hline 15 & 16 & NO & 0.0009 & 0.0094 & 0.171 \\
\hline 16 & 17 & $\mathrm{NO}$ & 0.0007 & 0.0089 & 0.1342 \\
\hline 16 & 19 & $\mathrm{NO}$ & 0.0016 & 0.0195 & 0.304 \\
\hline 16 & 21 & $\mathrm{NO}$ & 0.0008 & 0.0135 & 0.2548 \\
\hline 16 & 24 & NO & 0.0003 & 0.0059 & 0.068 \\
\hline 17 & 18 & $\mathrm{NO}$ & 0.0007 & 0.0082 & 0.1319 \\
\hline 17 & 27 & NO & 0.0013 & 0.0173 & 0.3216 \\
\hline 19 & 20 & YES & 0.0007 & 0.0138 & 0 \\
\hline 19 & 33 & YES & 0.0007 & 0.0142 & 0 \\
\hline 20 & 34 & YES & 0.0009 & 0.018 & 0 \\
\hline 21 & 22 & $\mathrm{NO}$ & 0.0008 & 0.014 & 0.2565 \\
\hline 22 & 23 & NO & 0.0006 & 0.0096 & 0.1846 \\
\hline 22 & 35 & YES & 0 & 0.0143 & 0 \\
\hline
\end{tabular}




\begin{tabular}{|r|r|l|r|r|r|}
\hline 23 & 24 & NO & 0.0022 & 0.035 & 0.361 \\
\hline 23 & 36 & YES & 0.0005 & 0.0272 & 0 \\
\hline 25 & 26 & NO & 0.0032 & 0.0323 & 0.513 \\
\hline 25 & 37 & YES & 0.0006 & 0.0232 & 0 \\
\hline 26 & 27 & NO & 0.0014 & 0.0147 & 0.2396 \\
\hline 26 & 28 & NO & 0.0043 & 0.0474 & 0.7802 \\
\hline 26 & 29 & NO & 0.0057 & 0.0625 & 1.029 \\
\hline 28 & 29 & NO & 0.0014 & 0.0151 & 0.249 \\
\hline 29 & 38 & YES & 0.0008 & 0.0156 & 0 \\
\hline
\end{tabular}

Table 4-3 IEEE 39 Bus Parameters- Generator Data

\begin{tabular}{|r|r|r|r|r|r|r|}
\hline Bus Number & \multicolumn{1}{|c|}{$\begin{array}{c}\text { Qgen } \\
{[\text { Pgen [MW] }}\end{array}$} & $\begin{array}{c}\text { V } \\
{[\mathrm{pu}]}\end{array}$ & \multicolumn{1}{c|}{$\begin{array}{c}\text { Pmax } \\
{[\mathrm{MW}]}\end{array}$} & $\begin{array}{c}\text { Qmin } \\
{[\text { MVAR] }}\end{array}$ & $\begin{array}{c}\text { Qmax } \\
{[\mathrm{MVAR}]}\end{array}$ \\
\hline 30 & 250 & 147.4 & 1.0475 & 1040 & -140 & 400 \\
\hline 31 & 520.87 & 199.55 & 0.982 & 646 & -100 & 300 \\
\hline 32 & 650 & 206.91 & 0.9831 & 725 & 150 & 300 \\
\hline 33 & 632 & 135.09 & 0.9972 & 652 & 0 & 250 \\
\hline 34 & 508 & 128.52 & 0.9972 & 508 & 0 & 167 \\
\hline 35 & 650 & 216.47 & 1.0493 & 687 & -100 & 300 \\
\hline 36 & 560 & 103.48 & 1.0635 & 580 & 0 & 240 \\
\hline 37 & 540 & 1.05 & 1.0278 & 564 & 0 & 250 \\
\hline 38 & 830 & 23.67 & 1.0265 & 865 & -150 & 300 \\
\hline 39 & 1000 & 89.2 & 1.03 & 1100 & -100 & 300 \\
\hline
\end{tabular}

\subsection{Test Cases}

\subsubsection{Verification of Index Performance Using 10-Bus Case}

The Voltage Collapse Proximity Index (eq. 3.32) by Balamourougan et al. and the Voltage Stability Index (VSI) (eq. 3.25) by Gong are the indices chosen for analysis. The VCPI and VSI were chosen due to the fact that they are computationally different. The VCPI, being more computationally efficient, strictly relies on the admittance matrix voltage magnitude and angle. On the other hand the VSI uses Thevenin model based on the admittance matrix along with synchrophasor values. The performance of the indices will be verified on a small system before being applied to a larger test system. Table 4-4 
shows a summary of their ideal performances.

Table 4-4 Summary Of Selected Indices

\begin{tabular}{|c|c|c|c|}
\hline Publication & Index & $\begin{array}{c}\text { Stable } \\
\text { Condition }\end{array}$ & $\begin{array}{l}\text { Unstable } \\
\text { Condition }\end{array}$ \\
\hline [31] & $V C P I_{k t h \text { bus }}=\left|1-\frac{\sum_{\substack{m=1 \\
m \neq k}}^{N} V_{m}^{x}}{V_{k}}\right|$ & $V C P I \rightarrow 0$ & $V C P I \rightarrow 1$ \\
\hline [27] & $V \mathrm{VI}_{k}=\min \left(\frac{P_{\text {margin }}}{P_{\text {max }}}, \frac{Q_{\text {margin }}}{Q_{\text {max }}}, \frac{S_{\text {margin }}}{S_{\text {max }}}\right)$ & VSI $\rightarrow 1$ & $\mathrm{VSI} \rightarrow \mathbf{0}$ \\
\hline
\end{tabular}

In order to analyze the indices, various test cases need to be developed. These test cases should reflect possible real life situations. The following section describes several factors that will be considered in the test cases.

\subsubsection{Increase All Loads}

The most common situation in power system stability studies has been the case where the system generation can no longer supply the demand. This situation often occurs when the total load demand is under predicted. Due to the complex nature of loads, the representation of this situation can be carried out in many ways. For this study, the selected loads will be increased at constant power factor until the system collapses.

\subsubsection{Reactive Load Increase}

As previously mentioned reactive power is closely linked to voltage. The more reactive-power demand, the more system losses there are, resulting in various instability issues. For this study, only the reactive power at the selected loads will be increased until the system collapses. 


\subsubsection{Contingency}

In actual power systems the network undergoes several changes in topology as a result of forced or unexpected outages. Certain lines within the system are very sensitive to changes in power flow, while others may be able to handle excess flow. Often times forced outages of specific lines can redirect power flow towards more lightly loaded lines and allow alleviation overloaded lines while still supplying the power demanded by all loads. During high demand times, loss of certain transmission lines can result in other lines to become overloaded and may lead to abnormal operating conditions. It is therefore useful to consider various contingencies when performing voltage stability analysis to determine the effects of increased system loading as well as the lines that are most likely to overload.

\subsubsection{Intermittent Generation}

The intermittent nature of wind generation has brought new challenges to the planning and operation of interconnected power systems with high penetration of grid connected wind farms. Therefore it is possible that the output power of a wind turbine can be significantly lower than the actual installed capacity. This deviation can lead to potential fluctuations in system frequency and voltage causing power system behavior to be more complex. Detailed studies that consider the various effects of wind generation to supply electricity demand are presented in [32-33]. The model chosen to represent the intermittent nature of wind is based on the aggregation of several wind turbines, which is elaborated in the following section. 


\subsubsection{Wind Farm Aggregation}

In order to harvest the most electrical energy from wind, many wind turbines are installed on the same location called "wind farms". As a result a large wind farm can have several hundreds of wind turbines covering an area of hundreds of square miles. Modeling of each individual unit for power systems analysis may result in large computation times may require extensive processing power. To overcome this issue, the wind turbines may be reduced to an equivalent single wind turbine generation unit. Aggregation techniques of variable speed wind turbines have been thoroughly discussed and their significance described in [34]. Studies comparing the results between detailed and aggregated models conclude that an aggregated electrical system and non-aggregated mechanical system is an efficient and accurate model for mid and long term simulations. [35]

Considering the aforementioned, the following test cases have been formulated for the IEEE 39-Bus system:

Case 1: Starting from a steady state base case, the real power (MW) will be increased at constant PF for all loads by $5 \%$ of base case until the system collapses.

Case 2: Starting from a steady state base case, the reactive power will be increase (MVAR) for all loads by 5\% of base case until the system collapses.

Case 3: Starting from a steady state base case, the real power (MW) will be increased at constant power factor by $2.5 \%$ of base case. In between 
two load increases a line will be removed to simulate a line tripping. Load demand will continue to increase at the same rate until the system collapses.

Case 4: Starting from a steady state base case, the system will be partitioned into areas. A load will be increased by $66 \%$ of its initial base load. The generation in that area will be adjusted to simulate various wind scenarios. 


\section{CHAPTER 5 SIMULATION AND RESULTS}

\subsection{BPA 10-Bus: Verification of Index Performance}

To verify the performance of each index, a series of load flows were conducted on PowerWorld Simulator. The synchrophasor variables: voltage magnitude, voltage angle, load powers and admittance matrix after each load increase were then outputted to MATLAB, which was then used to determine the VCPI at the bus. The algorithm for obtaining the VCPI and VSI are show in figures 5.1-5.2 respectively. 


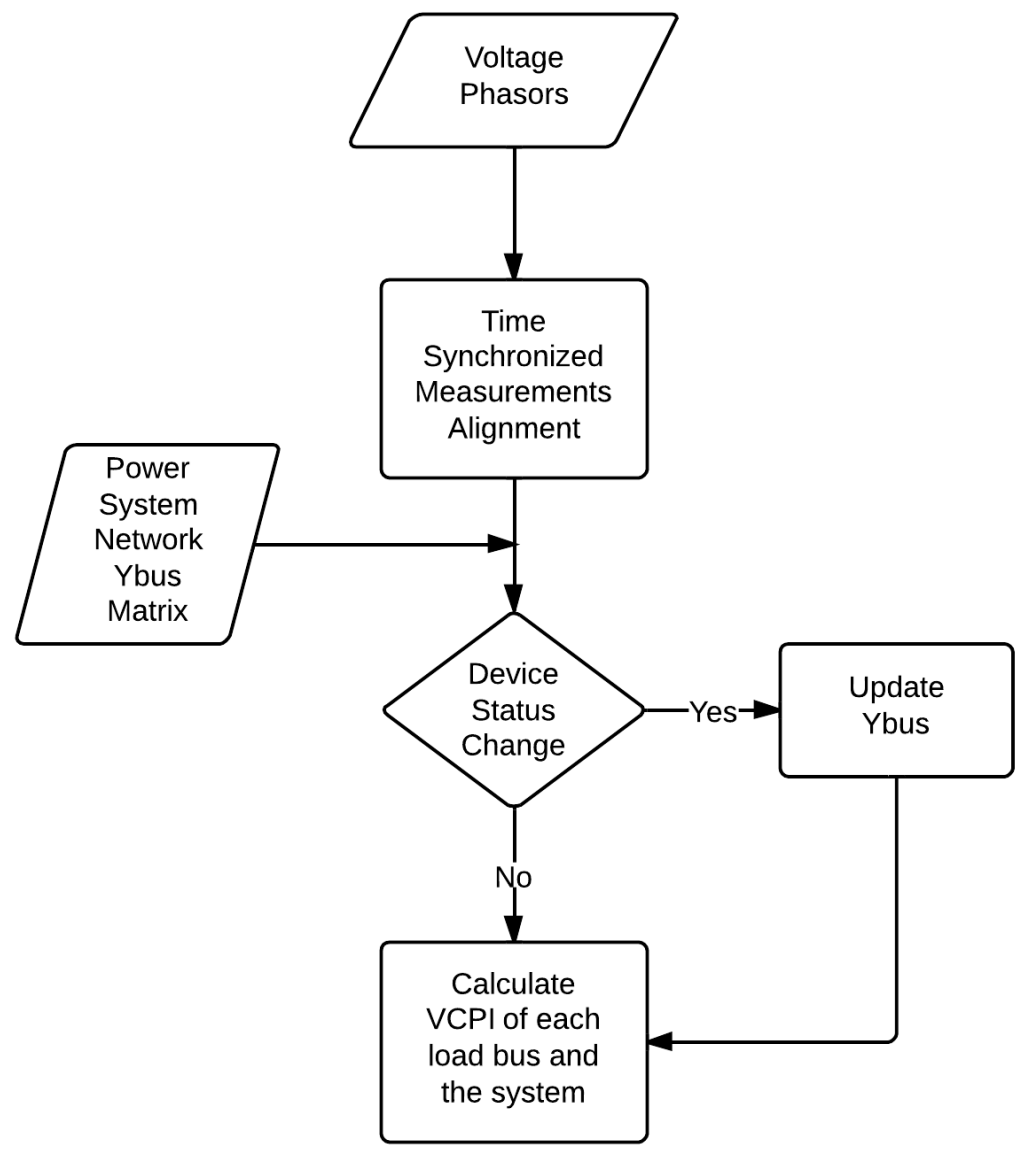

Figure 5.1 Algorithm for Computing VCPI 


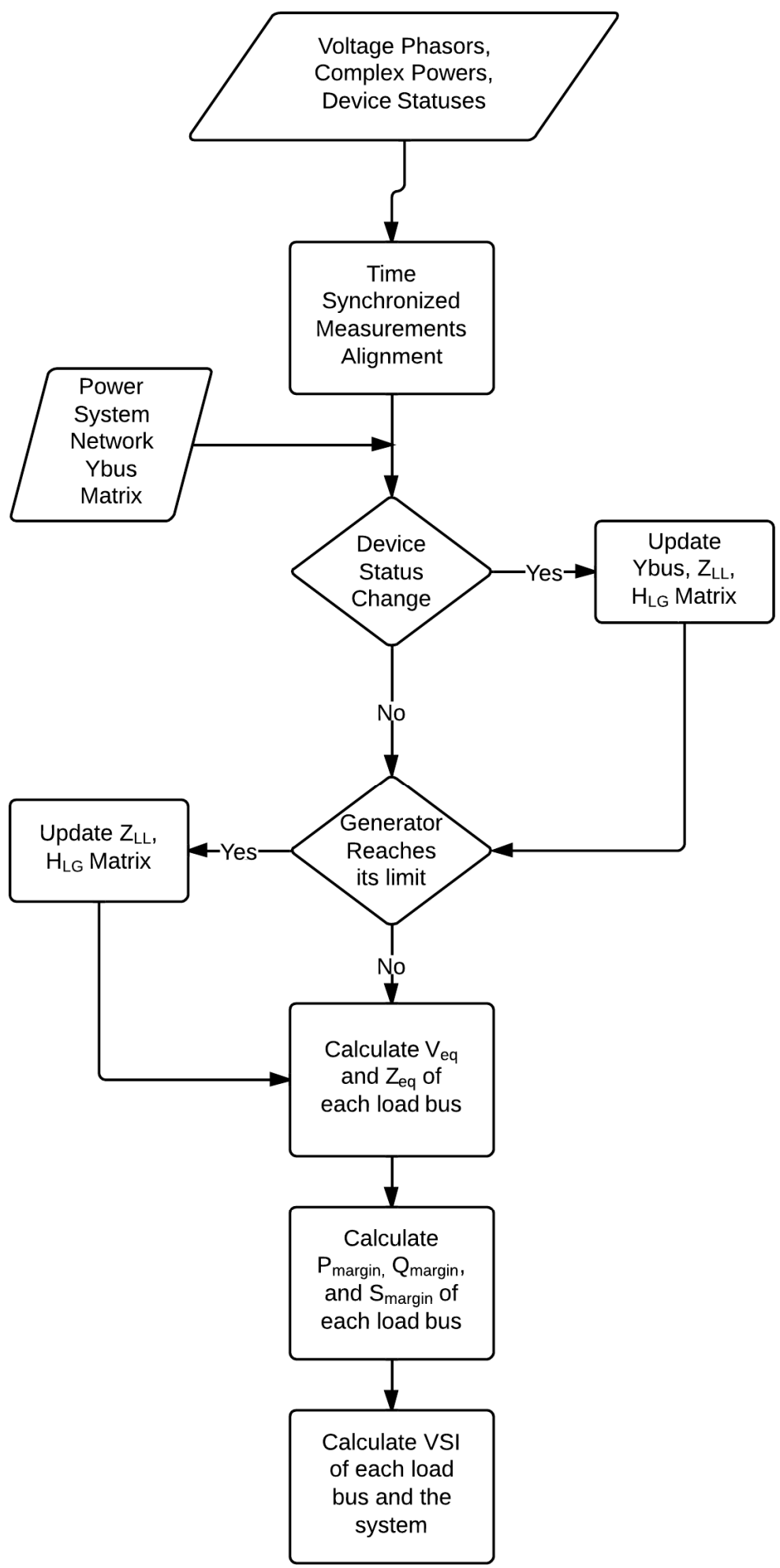

Figure 5.2 Algorithm for Computing VSI 
A selected bus in the 10-bus system was subject to real power increase at $100 \mathrm{MW}$ increments and constant power factor until the simulation diverged. The VSI and VCPI were plotted for each step increase. Bus 7 was chosen at random to be the bus for load increase. It is observed that the system collapses when the MW flow at bus 7 reaches $3200 \mathrm{MW}$

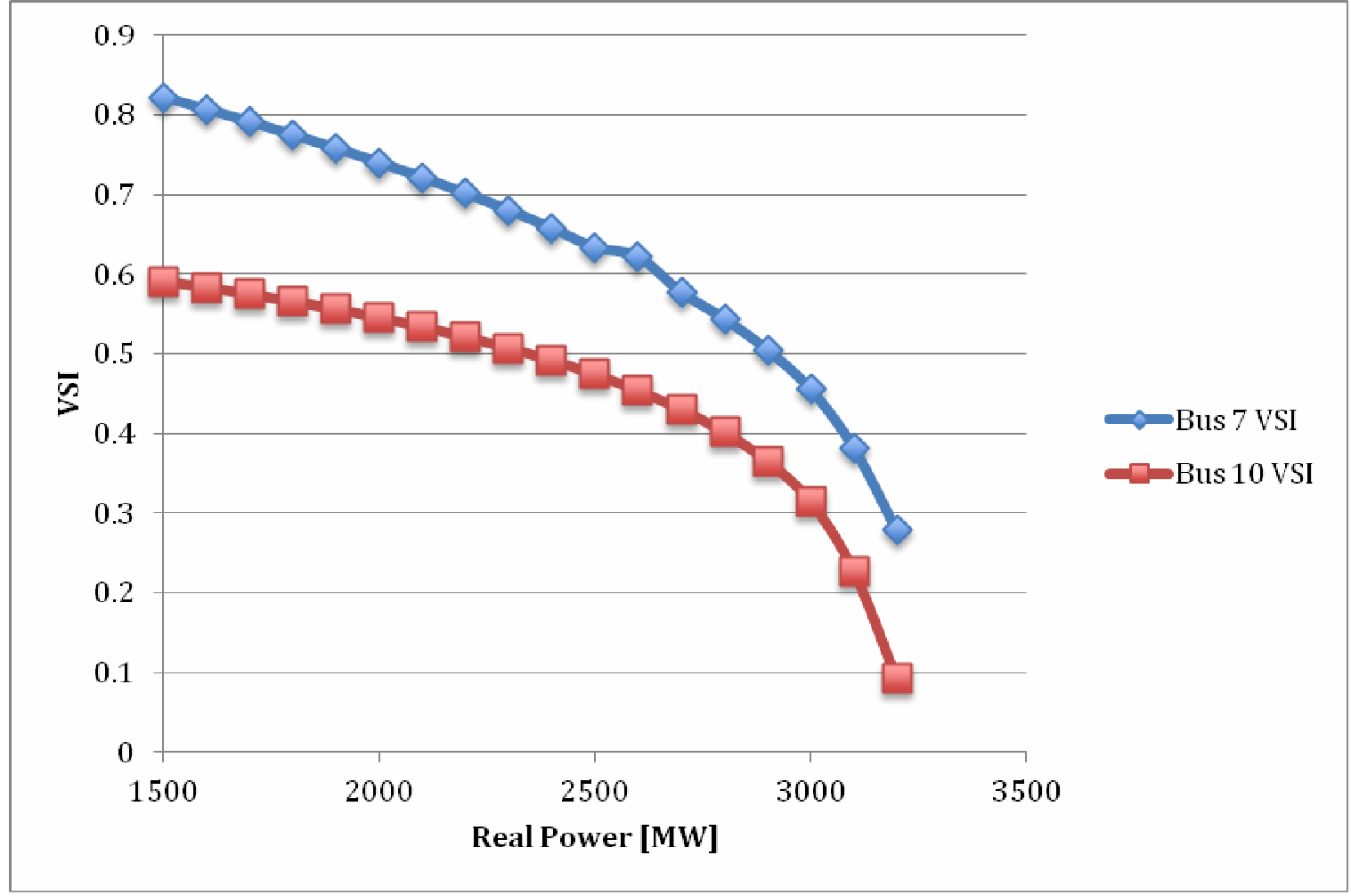

Figure 5.3 VSI for 10-bus system 


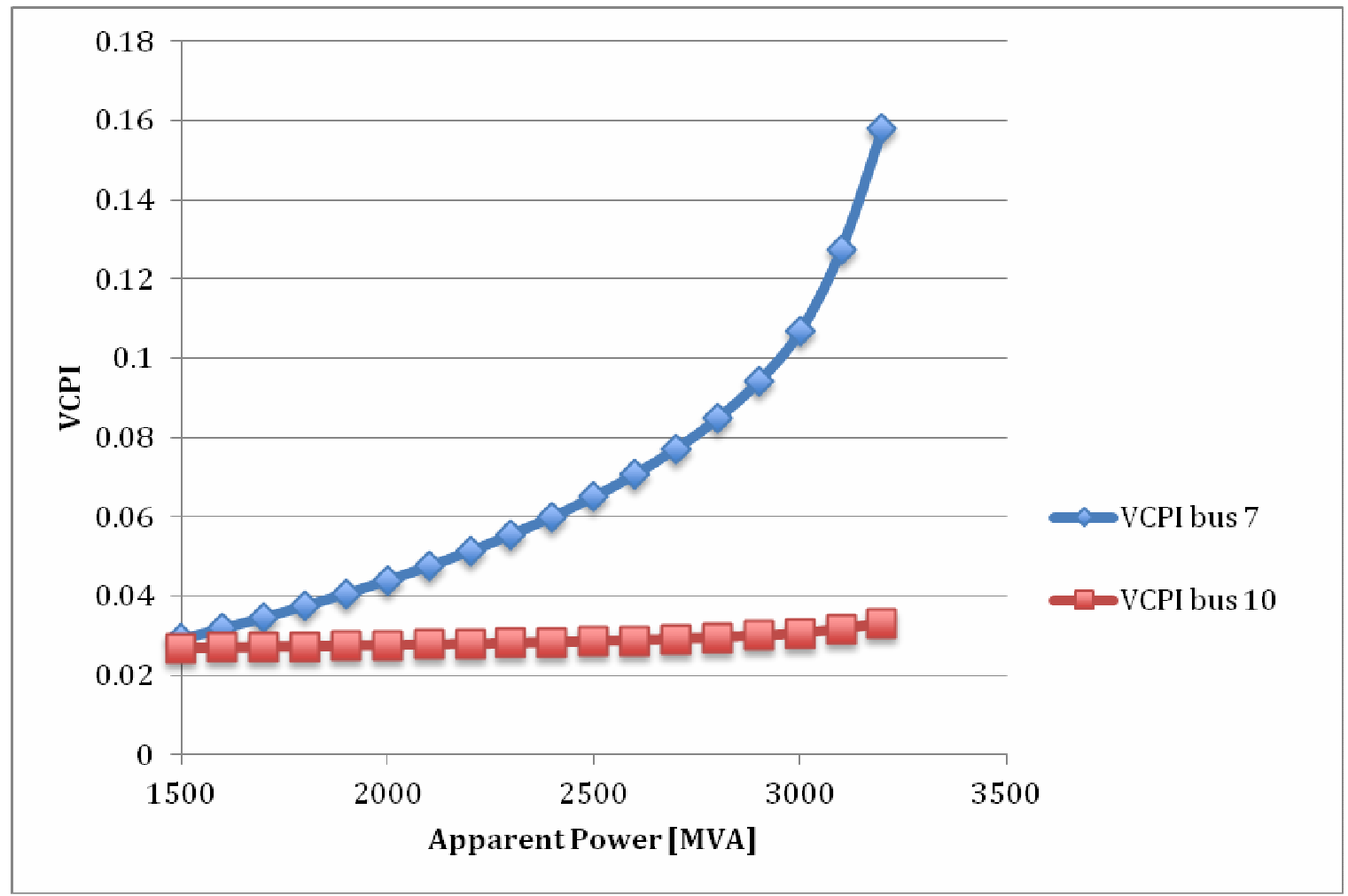

Figure 5.4 VCPI for 10-bus system

The steady state values for each index are shown on figures 5.3 and 5.4. For the base case the load real power was $1500 \mathrm{MW}$. The VSI and VCPI were .822 and .03 respectively.

From figure 5.3 it is observed that the VSI decreases towards zero when the load demand at bus 7 increases, which agrees with [27]. The values also range between 1 and 0 while under stable conditions. Figure 5.4 shows that as the real power load demand increases the corresponding VCPI increases towards 1, which is consistent with [31]. Also, while the system is stable the VCPI remains between 0 and 1 . When the system collapses the index displays a value of 0.16 . From figures 5.3 and 5.4 , it is shown that even though the indices are consistent with their theoretical formulas, they are not consistent with each other. The VSI shows that bus 10 approaches instability limits before bus 7, whereas the VCPI shows the opposite. The curves for both buses on figure 
5.3 follow a similar decreasing pattern. Whereas the slope for the VCPI on bus 7 increases at a faster rate for each load increase compared to bus 10. From the VCPI perspective, a load increase on bus 7 will only show noticeable index changes on bus 7 . On the other hand, from the VSI perspective it is seen that a load increase on bus 7 will affect the maximum power delivered and therefore the margins on bus 10. This suggests a stressed bus may not necessarily be the bus contributing to instability.

From this small system both indices are consistent with theoretical formulas; further investigation will be carried out with the larger 39-bus system in order to identify other characteristics as well as their application in voltage stability analysis.

\subsection{Case 1: IEEE 39-Bus: Increase all loads}

The loads in the 39-bus system were subject to real power increase at constant power factor. Each load was increased by $5 \%$ of the initial base case until the system collapsed, which is observed when the load is increase by 1.35 times the base case. Table 5-1 shows the total loads for each case.

Table 5-1 Load Factor with Corresponding Total System Laod

\begin{tabular}{|c|c|c|c|c|c|c|c|}
\hline Load & Base & 1.05 & 1.1 & 1.15 & 1.2 & 1.25 & 1.3 \\
Factor & Case & & & & & & \\
\hline $\begin{array}{c}\text { Load } \\
\text { Demand } \\
{[\mathrm{MW}]}\end{array}$ & 5278.9 & 5521.1 & 5784.0 & 6046.9 & 6309.8 & 6572.7 & 6835.6 \\
\hline
\end{tabular}

Initially the system is operating under base case conditions. Upon increasing the system load to 1.10 times the base case, generator 31 is the first to reach its reactive limit. Even 
though it is declared as the swing bus, the VSI algorithm is dependent on device status and must be updated. When the system load is increased by 1.20 times the base case, generators at buses 32 and 34 reach their reactive limits and can no longer sustain their scheduled voltages. Upon the next load increase, $1.25 \%$ of the base case, generators at buses 33 and 35 reach their limits as well. Finally the system load is increased again by $5 \%$ of its initial base case and the system can no longer maintain its stability, resulting in a system collapse. The VSI and VCPI for each load increase are tabulated in tables 5-2 and 5-3.

Table 5-2: Load Bus VSI for Various Load Factors

\begin{tabular}{|r|c|c|c|c|c|c|c|}
\hline \multicolumn{1}{|c|}{ Bus \# } & $\mathbf{1}$ & $\mathbf{1 . 0 5}$ & $\mathbf{1 . 1}$ & $\mathbf{1 . 1 5}$ & $\mathbf{1 . 2}$ & $\mathbf{1 . 2 5}$ & $\mathbf{1 . 3}$ \\
\hline $\mathbf{3}$ & 0.926 & 0.922 & 0.914 & 0.909 & 0.891 & 0.866 & 0.585 \\
\hline $\mathbf{4}$ & 0.833 & 0.823 & 0.781 & 0.766 & 0.661 & 0.580 & 0.104 \\
\hline $\mathbf{7}$ & 0.905 & 0.899 & 0.861 & 0.850 & 0.778 & 0.729 & 0.293 \\
\hline $\mathbf{8}$ & 0.804 & 0.792 & 0.721 & 0.701 & 0.576 & 0.497 & 0.018 \\
\hline $\mathbf{1 2}$ & 0.894 & 0.887 & 0.872 & 0.863 & 0.804 & 0.765 & 0.539 \\
\hline $\mathbf{1 5}$ & 0.868 & 0.860 & 0.848 & 0.839 & 0.802 & 0.708 & 0.326 \\
\hline $\mathbf{1 6}$ & 0.939 & 0.935 & 0.930 & 0.926 & 0.912 & 0.831 & 0.494 \\
\hline $\mathbf{1 8}$ & 0.949 & 0.946 & 0.942 & 0.938 & 0.929 & 0.906 & 0.745 \\
\hline $\mathbf{2 0}$ & 0.841 & 0.833 & 0.825 & 0.816 & 0.588 & 0.262 & 0.056 \\
\hline $\mathbf{2 1}$ & 0.901 & 0.896 & 0.890 & 0.884 & 0.873 & 0.771 & 0.416 \\
\hline $\mathbf{2 3}$ & 0.935 & 0.932 & 0.928 & 0.925 & 0.920 & 0.857 & 0.457 \\
\hline $\mathbf{2 4}$ & 0.925 & 0.921 & 0.916 & 0.911 & 0.899 & 0.830 & 0.508 \\
\hline $\mathbf{2 5}$ & 0.946 & 0.943 & 0.940 & 0.937 & 0.933 & 0.927 & 0.852 \\
\hline $\mathbf{2 6}$ & 0.951 & 0.949 & 0.946 & 0.943 & 0.938 & 0.930 & 0.882 \\
\hline $\mathbf{2 7}$ & 0.876 & 0.870 & 0.862 & 0.854 & 0.841 & 0.809 & 0.640 \\
\hline $\mathbf{2 8}$ & 0.901 & 0.896 & 0.891 & 0.885 & 0.879 & 0.871 & 0.849 \\
\hline $\mathbf{2 9}$ & 0.924 & 0.920 & 0.916 & 0.912 & 0.908 & 0.902 & 0.889 \\
\hline Global Index & 0.804 & 0.792 & 0.721 & 0.701 & 0.576 & 0.262 & 0.018 \\
\hline & & & & & & & \\
\hline
\end{tabular}


Table 5-3 Load Bus VCPI for Various Load Factors

\begin{tabular}{|c|c|c|c|c|c|c|c|}
\hline Bus \# Factor & 1 & 1.05 & 1.1 & 1.15 & 1.2 & 1.25 & 1.3 \\
\hline 3 & 0.163 & 0.172 & 0.181 & 0.192 & 0.205 & 0.223 & 0.267 \\
\hline 4 & 0.258 & 0.274 & 0.293 & 0.315 & 0.346 & 0.394 & 0.534 \\
\hline 7 & 0.076 & 0.081 & 0.087 & 0.094 & 0.104 & 0.120 & 0.168 \\
\hline 8 & 0.164 & 0.175 & 0.187 & 0.202 & 0.224 & 0.258 & 0.358 \\
\hline 12 & 0.134 & 0.146 & 0.160 & 0.177 & 0.203 & 0.244 & 0.371 \\
\hline 15 & 0.220 & 0.233 & 0.248 & 0.264 & 0.285 & 0.315 & 0.401 \\
\hline 16 & 0.061 & 0.064 & 0.067 & 0.071 & 0.076 & 0.082 & 0.100 \\
\hline 18 & 0.076 & 0.080 & 0.085 & 0.090 & 0.096 & 0.104 & 0.125 \\
\hline 20 & 0.662 & 0.687 & 0.713 & 0.740 & 0.770 & 0.812 & 0.957 \\
\hline 21 & 0.186 & 0.197 & 0.208 & 0.220 & 0.233 & 0.252 & 0.301 \\
\hline 23 & 0.137 & 0.145 & 0.152 & 0.160 & 0.169 & 0.180 & 0.206 \\
\hline 24 & 0.155 & 0.163 & 0.172 & 0.181 & 0.193 & 0.208 & 0.249 \\
\hline 25 & 0.141 & 0.147 & 0.153 & 0.160 & 0.167 & 0.175 & 0.190 \\
\hline 26 & 0.125 & 0.128 & 0.132 & 0.136 & 0.141 & 0.147 & 0.159 \\
\hline 27 & 0.211 & 0.223 & 0.236 & 0.249 & 0.265 & 0.286 & 0.331 \\
\hline 28 & 0.217 & 0.228 & 0.240 & 0.251 & 0.264 & 0.278 & 0.301 \\
\hline 29 & 0.218 & 0.225 & 0.232 & 0.240 & 0.248 & 0.257 & 0.270 \\
\hline Global Index & 0.662 & 0.687 & 0.713 & 0.740 & 0.770 & 0.812 & 0.957 \\
\hline
\end{tabular}

To illustrate an overall pattern, the VSIs that showed the lowest index before collapsing are plotted on figure 5.5. Once again, a collapse is defined when the load flow diverges. From figure 5.5, the VSI follows a linear trend for the first several load increase, which is until the reactive capability of generator 1 has been exceeded. Beyond this point, each index shows various nonlinearities. At base case conditions, Bus 7 shows the highest signs of stability with an index of 0.89 . Bus 20 shows the most sensitive reactions to increases in load with the lowest value before diverging being 0.26 


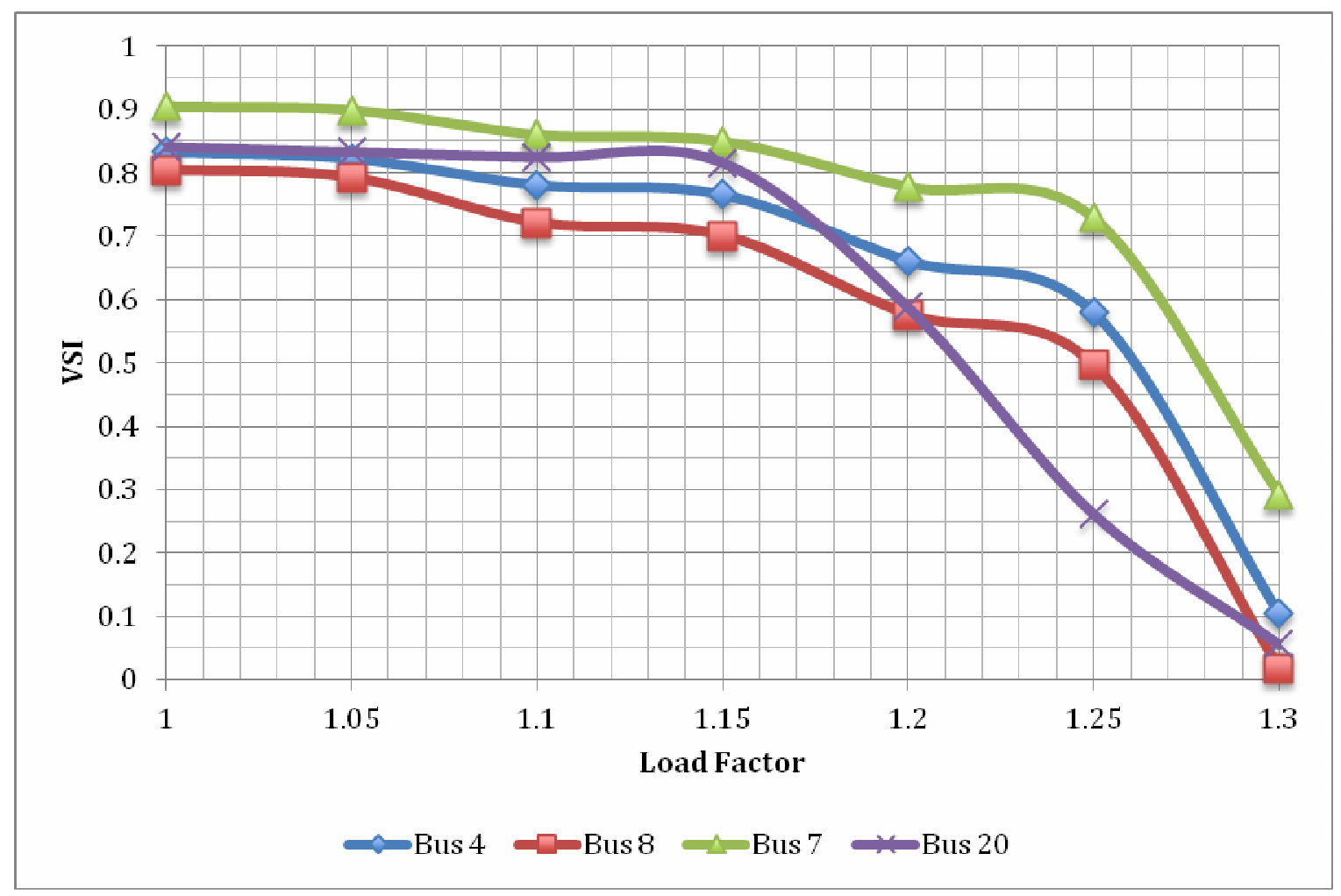

Figure 5.5 VSI for Load Buses 4, 7, 8, 20

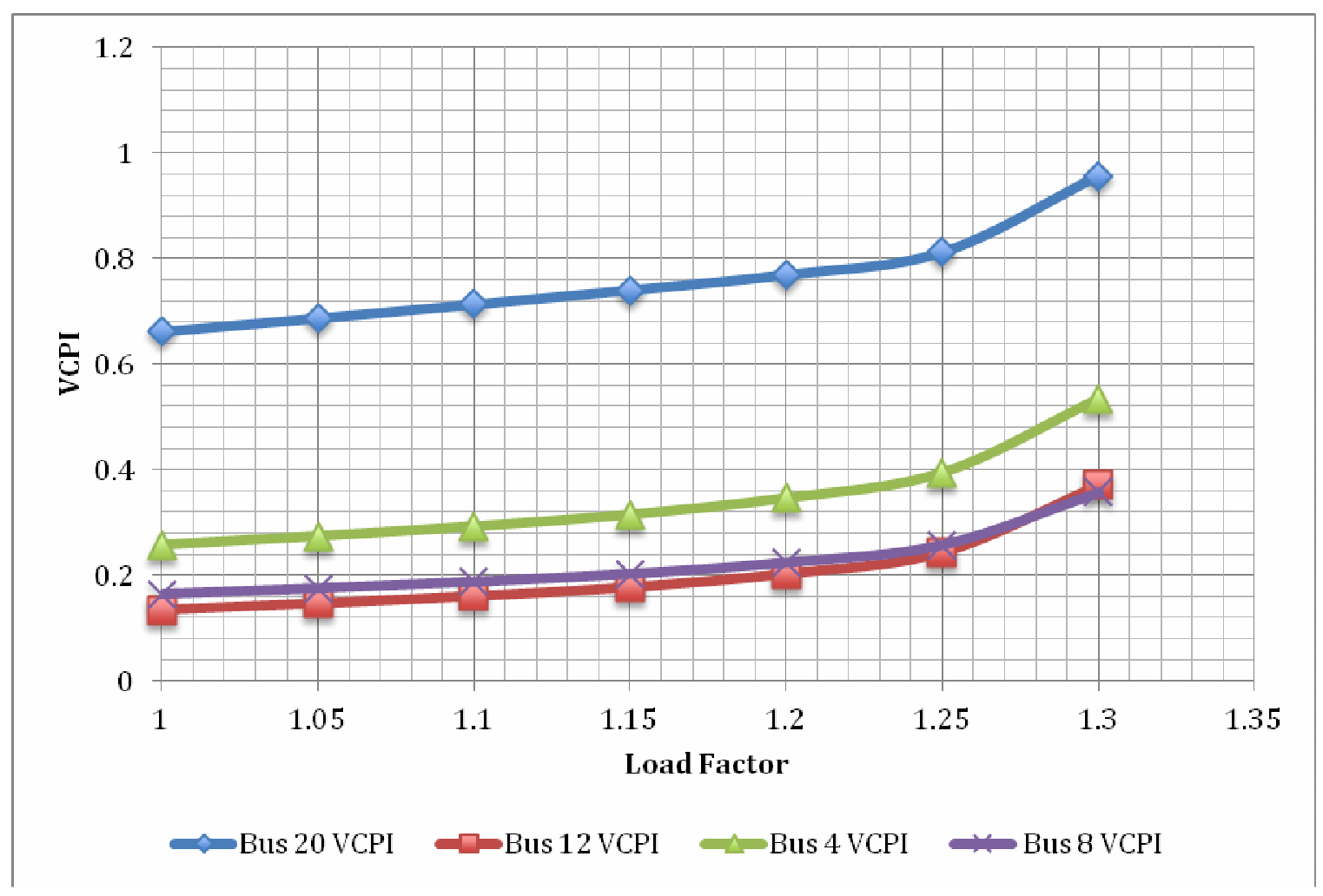

Figure 5.6 VCPI for Load Buses 4, 7, 8, 20 
For the VCPI plot as shown on figure 5.6, the indices follow a linear trend as the system load increases. When the load factor exceeds 1.25 , the slope is much steeper before reaching its instability limit. The fact that the VSI shows a value close to 0 right before collapse reveals that the system is at its marginal stability limit and that the method can accurately determine the state of the system. On the other hand, it can be seen that none of the VCPIs reach unity near voltage collapse, as it should. The highest index calculated was .81 from bus 20 . It is when the system collapses, that a value of .91 from bus 20 is revealed.

So far it is shown that for even a large system, both indices approach their respective theoretical limits, thus indicating that both indices are capable of determining voltage instability. In addition to the calculated indices, load bus voltage magnitudes were monitored for each load increase. Table 5-4 shows that the load bus voltage magnitudes decrease after each load increase, as expected.

Table 5-4 Load Bus Voltages, Case 1

\begin{tabular}{|c|c|c|c|c|c|c|c|}
\hline Factor & & & & & & & \\
\hline 3 & Base & 1.05 & 1.10 & 1.15 & 1.2 & 1.25 & 1.3 \\
\hline 4 & 1.030 & 1.027 & 1.022 & 1.015 & 1.003 & 0.981 & 0.933 \\
\hline 7 & 1.003 & 0.997 & 0.988 & 0.975 & 0.950 & 0.910 & 0.818 \\
\hline 8 & 0.997 & 0.989 & 0.979 & 0.963 & 0.935 & 0.890 & 0.777 \\
\hline 12 & 0.996 & 0.988 & 0.978 & 0.963 & 0.935 & 0.891 & 0.779 \\
\hline 15 & 1.000 & 0.993 & 0.984 & 0.969 & 0.940 & 0.893 & 0.787 \\
\hline 16 & 1.014 & 1.010 & 1.004 & 0.996 & 0.980 & 0.952 & 0.897 \\
\hline 18 & 1.030 & 1.028 & 1.024 & 1.018 & 1.008 & 0.988 & 0.952 \\
\hline 20 & 0.982 & 1.027 & 1.023 & 1.016 & 1.005 & 0.984 & 0.943 \\
\hline 21 & 1.031 & 1.028 & 1.025 & 1.021 & 1.013 & 0.996 & 0.965 \\
\hline 23 & 1.044 & 1.043 & 1.041 & 1.038 & 1.034 & 1.022 & 1.003 \\
\hline 24 & 1.036 & 1.034 & 1.030 & 1.025 & 1.016 & 0.998 & 0.965 \\
\hline 25 & 1.057 & 1.055 & 1.052 & 1.048 & 1.043 & 1.033 & 1.016 \\
\hline 26 & 1.052 & 1.049 & 1.046 & 1.042 & 1.035 & 1.023 & 1.003 \\
\hline 27 & 1.037 & 1.034 & 1.030 & 1.024 & 1.015 & 0.999 & 0.971 \\
\hline 28 & 1.050 & 1.048 & 1.046 & 1.044 & 1.040 & 1.033 & 1.026 \\
\hline 29 & 1.050 & 1.049 & 1.047 & 1.045 & 1.043 & 1.038 & 1.036 \\
\hline & & & & & & & \\
\hline
\end{tabular}


When the base case system load is increased by a factor of 1.25 , Bus $4,7,8$ and 12 show the largest signs of low voltage problems, ranging from 0.09 to 0.11 pu drop. Considering that the nearby generator at buses 31 and 32 have reached their reactive limits at 1.2 times the base case, and therefore limiting the capacitive VAR support, the large voltage drop at buses 7,8 and 12 is expected. At this point the buses are still voltage stable, however any large disturbance such as large load increase or a loss of any major element can cause the system to lose its stability. Upon increasing the system load by a factor of 1.3 times the base case, buses 4, 7, 8 and 12 are well below nominal operating conditions and therefore collapsing.

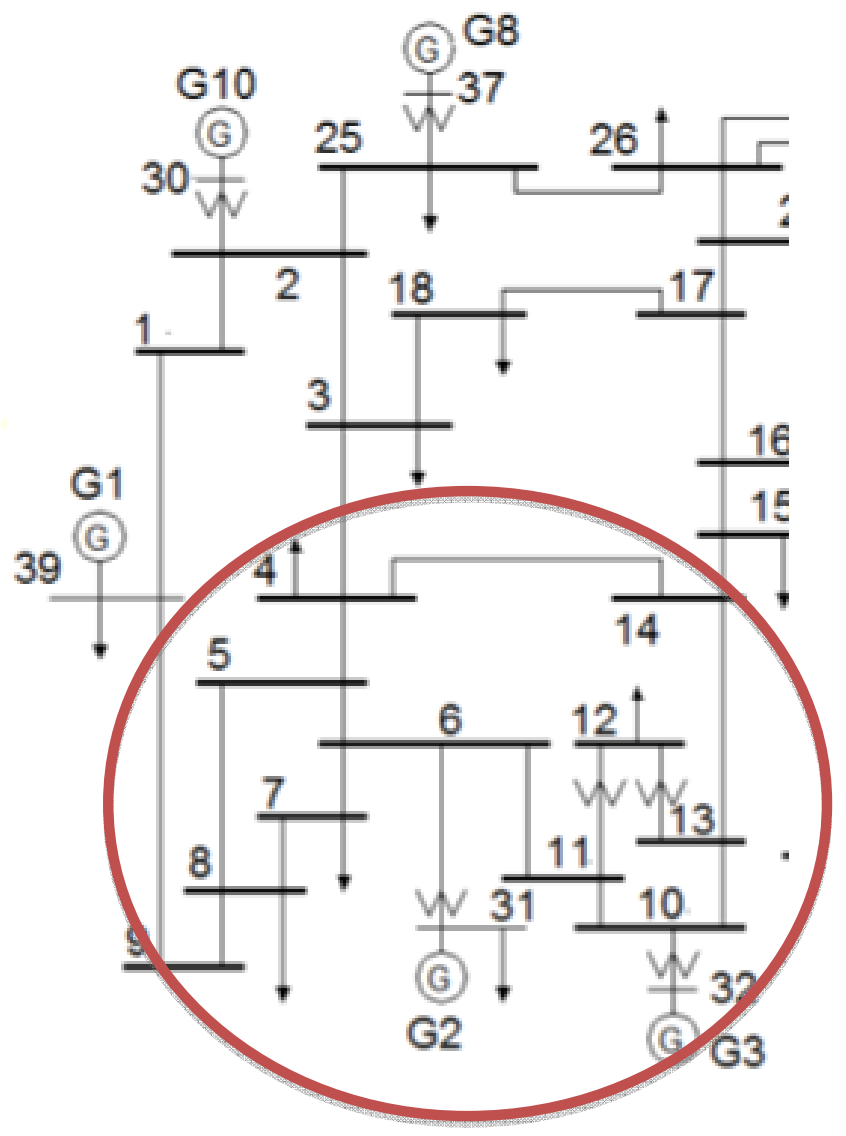

Figure 5.7 Load Buses with respect to nearest generation units 
Chapter 3 has mentioned voltage magnitudes alone do not give a good indication of proximity to voltage stability limit, especially for a system with high reactive power compensation. However in most situations, systems are often lacking reactive power resulting in low voltages when load is increased. By analyzing and comparing bus voltages with index values, it is possible to determine whether low voltages or even voltages alone are capable of determining whether the system is stable or not.

Indices and voltage magnitudes for buses 8 and 12 are plotted for each load increase as shown in figures 5.8-5.9. These buses are significant since they show the lowest voltage magnitudes before collapse. When the system load is increased by 1.25 times the base case, it is seen that even though these two buses experience about $.1 \mathrm{pu}$ reduction in voltage, both indices indicate that bus 12 is voltage stable and bus 8 can be either stable or unstable, depending on where the margin of instability is placed. Similarly, the load bus having the next lowest voltage, bus 7, also experiences voltage reduction at its terminals with only a minimal change in VSI and VCPI values indicating that they are voltage stable. 


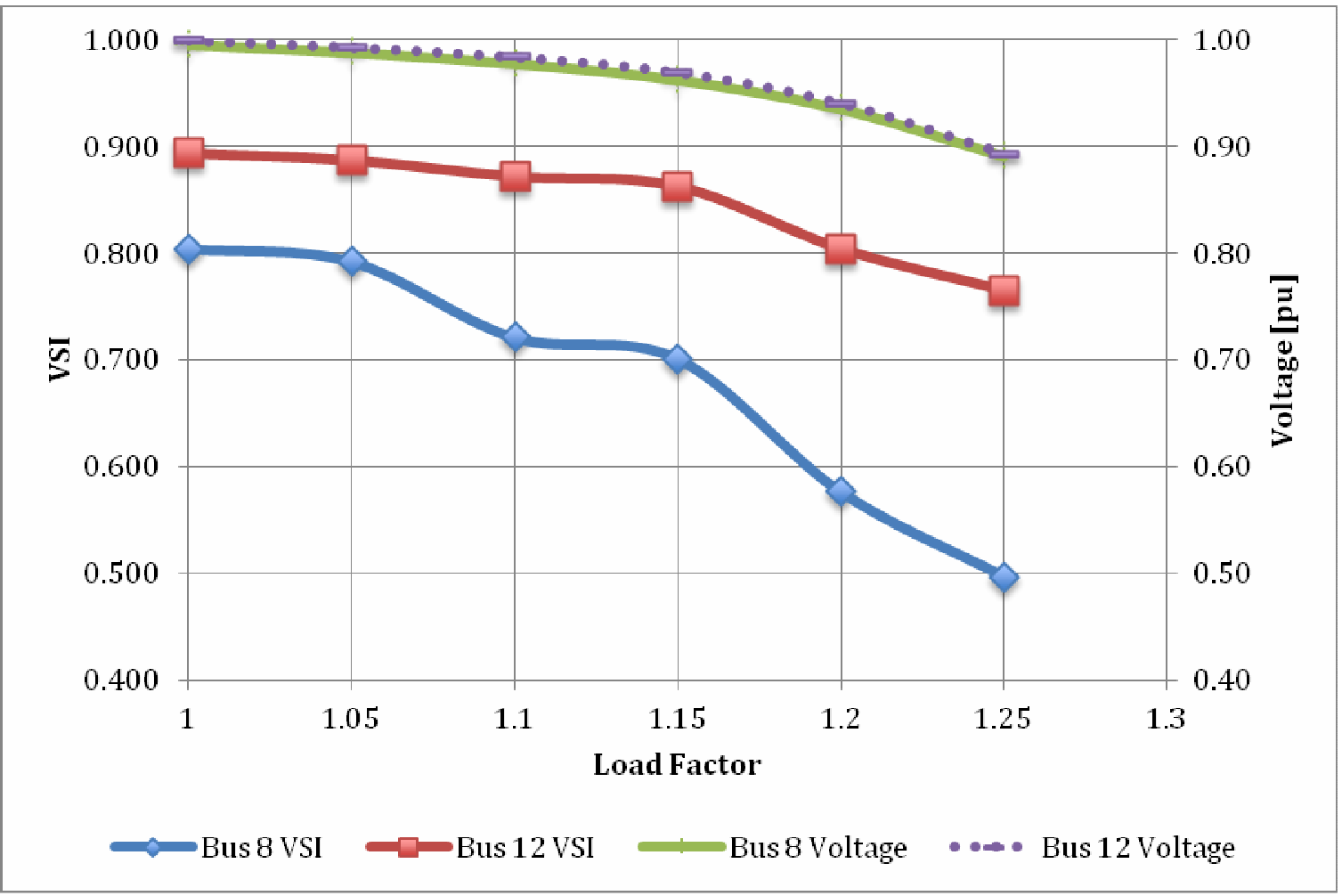

Figure 5.8 VSI and Their Respected Bus Voltages

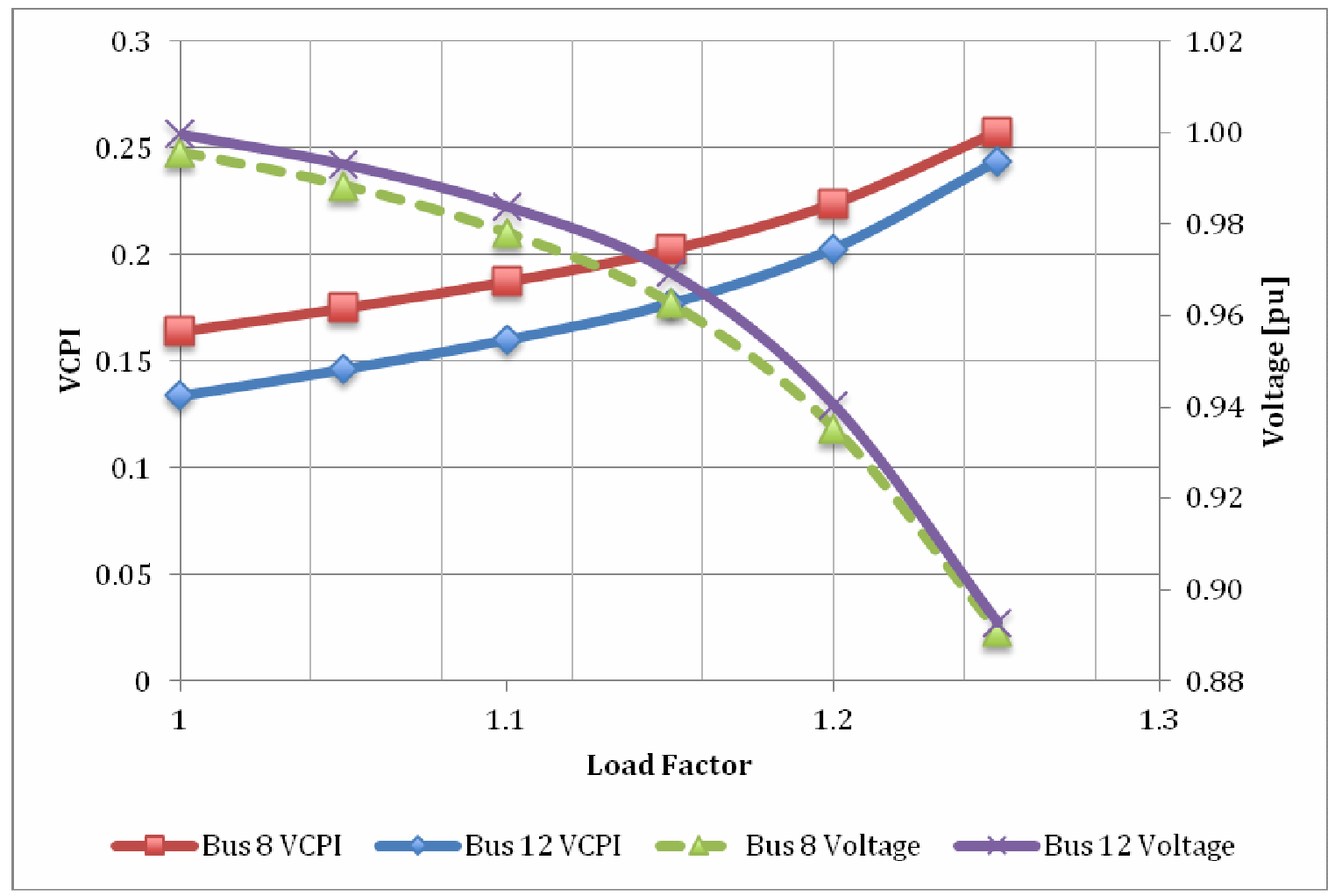

Figure 5.9 VCPI and Their Respected Bus Voltages 
It is clear that monitoring only the voltage at a bus might lead to a false prediction of an upcoming voltage collapse. By using indices that consider not only voltage at the buses, but also other relevant system parameters such as topology, determining the likelihood of a voltage collapse scenario is much more accurate.

\subsection{Case 2: 39 Bus - Reactive power case}

The loads in the 39-bus system were subject to reactive power increase. Each load was increased by $5 \%$ of the initial base case until the system collapsed. At 1.35 times the initial base case, the generator at bus 34 is the first to reach its reactive limit. By the time the total reactive demand reaches 1.8 times the initial base case the generators at buses $32,33,35,36$ reach their reactive limits and these buses suffer severe low voltage issues. The system finally collapses once the total reactive load was increased past 1.95 times the initial base case. The indices for selected buses are plotted in figures 5.10 and 5.11. The rest are tabulated in tables 5-5 through 5-8. 


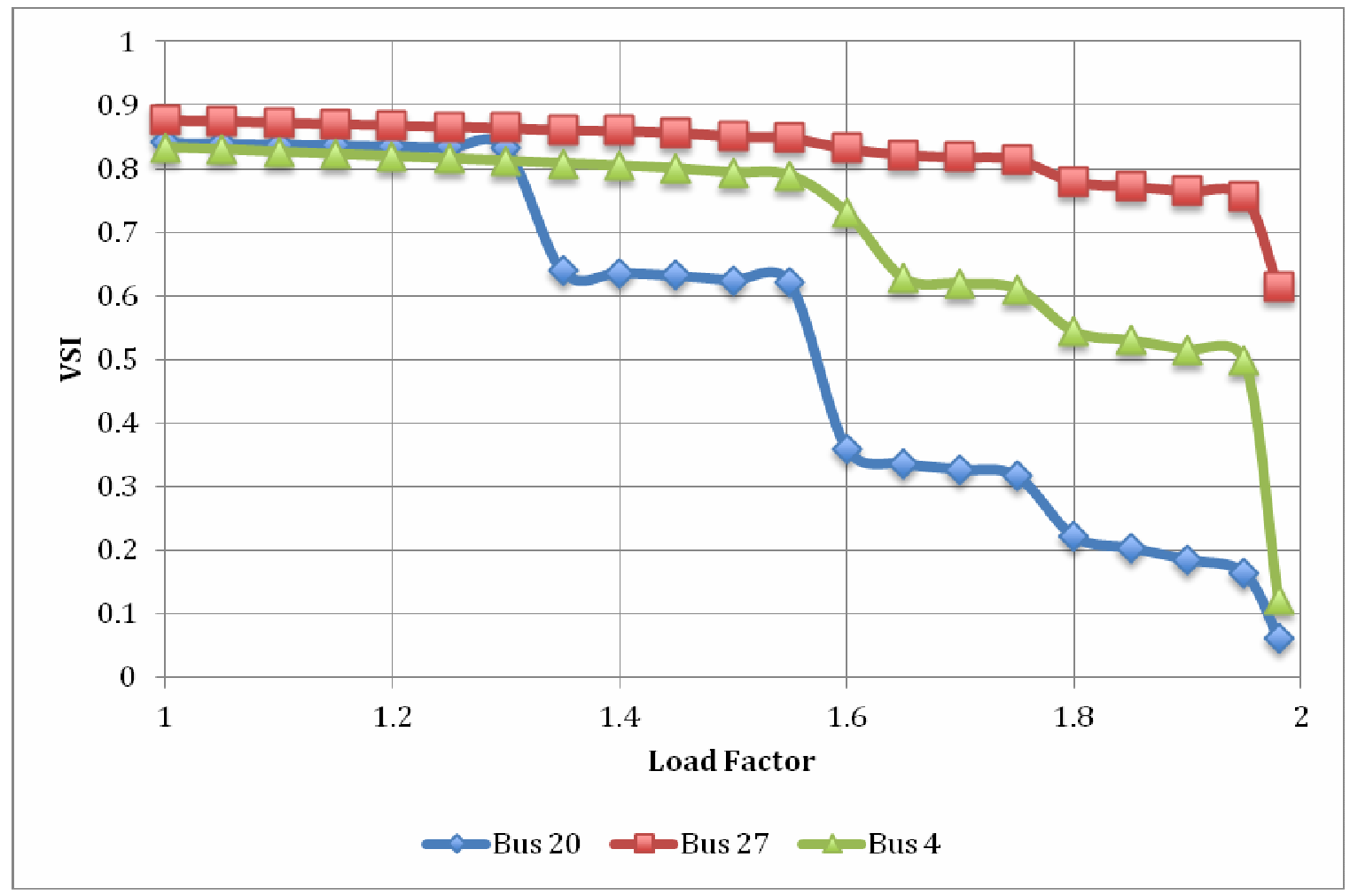

Figure 5.10 VSIs for Case 2: Bus 4, 20, and 27

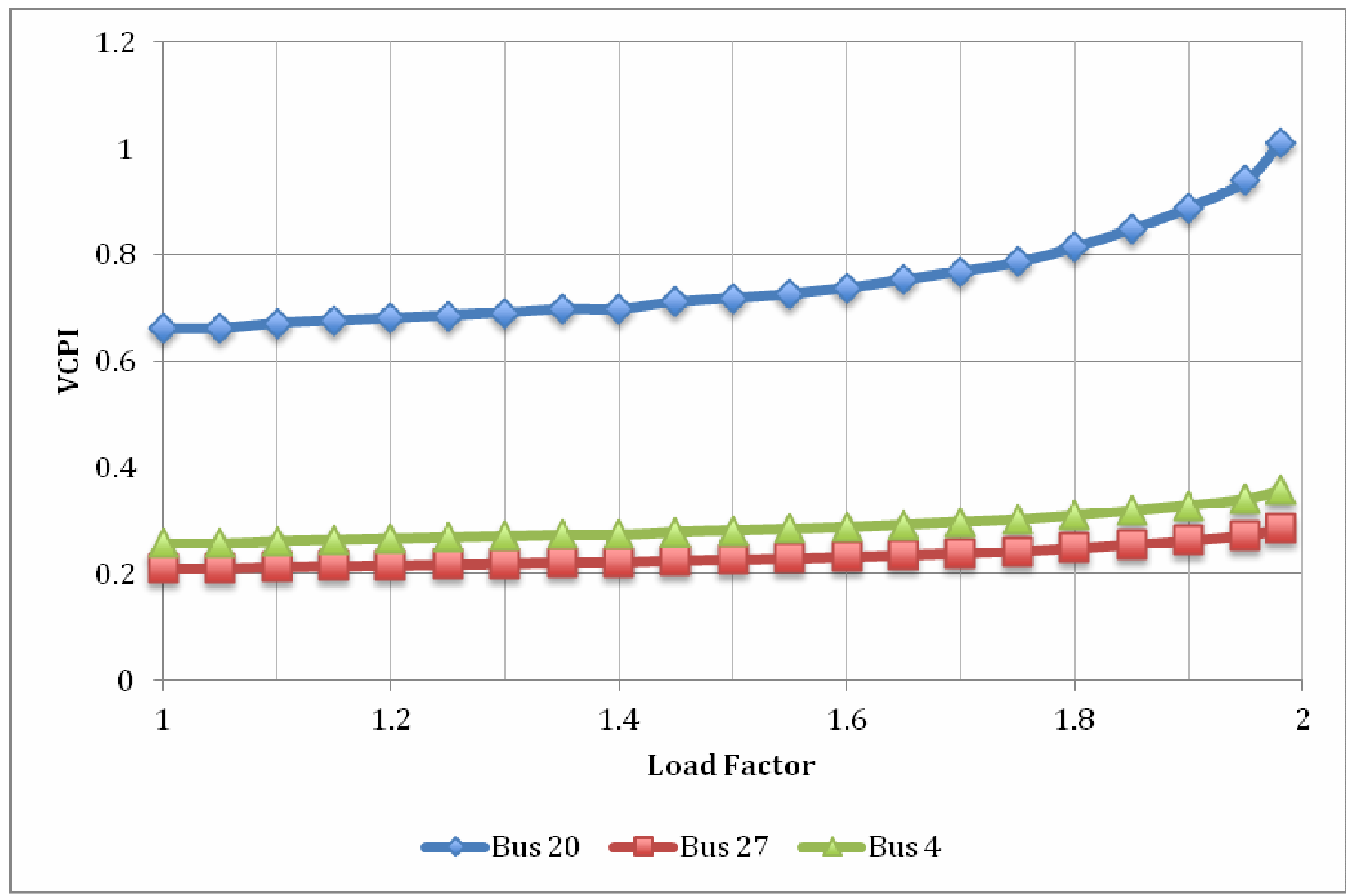

Figure 5.11 VCPIs for Case 2: Bus 4, 20, and 27 
Table 5-5 Load Bus VSI for Case 2

\begin{tabular}{|c|c|c|c|c|c|c|c|c|c|c|}
\hline Bus \# & 1 & 1.05 & 1.1 & 1.15 & 1.2 & 1.25 & 1.3 & 1.35 & 1.4 & 1.45 \\
\hline 3 & 0.926 & 0.926 & 0.925 & 0.925 & 0.925 & 0.924 & 0.924 & 0.923 & 0.923 & 0.923 \\
\hline 4 & 0.833 & 0.830 & 0.826 & 0.823 & 0.819 & 0.816 & 0.812 & 0.808 & 0.805 & 0.800 \\
\hline 7 & 0.905 & 0.904 & 0.902 & 0.900 & 0.898 & 0.896 & 0.893 & 0.891 & 0.890 & 0.887 \\
\hline 8 & 0.804 & 0.801 & 0.797 & 0.793 & 0.790 & 0.786 & 0.782 & 0.778 & 0.775 & 0.770 \\
\hline 12 & 0.894 & 0.888 & 0.882 & 0.876 & 0.871 & 0.865 & 0.859 & 0.853 & 0.848 & 0.841 \\
\hline 15 & 0.868 & 0.865 & 0.861 & 0.858 & 0.854 & 0.851 & 0.847 & 0.841 & 0.838 & 0.833 \\
\hline 16 & 0.939 & 0.938 & 0.938 & 0.937 & 0.936 & 0.936 & 0.935 & 0.932 & 0.932 & 0.931 \\
\hline 18 & 0.949 & 0.948 & 0.947 & 0.947 & 0.946 & 0.945 & 0.945 & 0.943 & 0.943 & 0.942 \\
\hline 20 & 0.841 & 0.840 & 0.838 & 0.837 & 0.835 & 0.834 & 0.832 & 0.639 & 0.636 & 0.632 \\
\hline 21 & 0.901 & 0.899 & 0.897 & 0.895 & 0.893 & 0.890 & 0.888 & 0.884 & 0.882 & 0.879 \\
\hline 23 & 0.935 & 0.934 & 0.933 & 0.932 & 0.931 & 0.929 & 0.928 & 0.927 & 0.925 & 0.924 \\
\hline 24 & 0.925 & 0.925 & 0.925 & 0.925 & 0.924 & 0.924 & 0.924 & 0.922 & 0.922 & 0.922 \\
\hline 25 & 0.946 & 0.945 & 0.945 & 0.944 & 0.943 & 0.943 & 0.942 & 0.942 & 0.941 & 0.940 \\
\hline 26 & 0.951 & 0.951 & 0.950 & 0.950 & 0.949 & 0.949 & 0.948 & 0.948 & 0.948 & 0.947 \\
\hline 27 & 0.876 & 0.875 & 0.872 & 0.870 & 0.868 & 0.866 & 0.864 & 0.861 & 0.859 & 0.857 \\
\hline 28 & 0.901 & 0.901 & 0.899 & 0.899 & 0.898 & 0.897 & 0.896 & 0.895 & 0.894 & 0.893 \\
\hline 29 & 0.924 & 0.924 & 0.923 & 0.923 & 0.922 & 0.922 & 0.921 & 0.921 & 0.921 & 0.920 \\
\hline
\end{tabular}

Table 5-6 Load Bus VSI for case 2(Cont.)

\begin{tabular}{|r|c|c|c|c|c|c|c|c|c|c|c|}
\hline Factor & & & & & & & & & & & \\
\hline & $\mathbf{1 . 5}$ & $\mathbf{1 . 5 5}$ & $\mathbf{1 . 6}$ & $\mathbf{1 . 6 5}$ & $\mathbf{1 . 7}$ & $\mathbf{1 . 7 5}$ & $\mathbf{1 . 8}$ & $\mathbf{1 . 8 5}$ & $\mathbf{1 . 9}$ & $\mathbf{1 . 9 5}$ & $\mathbf{1 . 9 8}$ \\
\hline $\mathbf{4}$ & 0.921 & 0.920 & 0.910 & 0.897 & 0.896 & 0.895 & 0.875 & 0.873 & 0.870 & 0.867 & 0.686 \\
\hline $\mathbf{7}$ & 0.884 & 0.882 & 0.855 & 0.771 & 0.766 & 0.760 & 0.730 & 0.722 & 0.713 & 0.703 & 0.412 \\
\hline $\mathbf{8}$ & 0.765 & 0.761 & 0.712 & 0.569 & 0.561 & 0.552 & 0.503 & 0.491 & 0.478 & 0.463 & 0.081 \\
\hline $\mathbf{1 2}$ & 0.834 & 0.828 & 0.784 & 0.727 & 0.716 & 0.705 & 0.668 & 0.652 & 0.635 & 0.616 & 0.399 \\
\hline $\mathbf{1 5}$ & 0.817 & 0.812 & 0.750 & 0.710 & 0.701 & 0.692 & 0.570 & 0.551 & 0.530 & 0.505 & 0.217 \\
\hline $\mathbf{1 6}$ & 0.918 & 0.917 & 0.874 & 0.859 & 0.856 & 0.854 & 0.759 & 0.751 & 0.741 & 0.729 & 0.531 \\
\hline $\mathbf{1 8}$ & 0.938 & 0.938 & 0.926 & 0.917 & 0.916 & 0.914 & 0.888 & 0.885 & 0.880 & 0.876 & 0.756 \\
\hline $\mathbf{2 0}$ & 0.625 & 0.621 & 0.360 & 0.336 & 0.326 & 0.316 & 0.221 & 0.203 & 0.184 & 0.163 & 0.071 \\
\hline $\mathbf{2 1}$ & 0.832 & 0.828 & 0.787 & 0.769 & 0.763 & 0.756 & 0.580 & 0.560 & 0.538 & 0.511 & 0.283 \\
\hline $\mathbf{2 3}$ & 0.887 & 0.885 & 0.868 & 0.861 & 0.857 & 0.854 & 0.607 & 0.589 & 0.569 & 0.545 & 0.355 \\
\hline $\mathbf{2 4}$ & 0.909 & 0.909 & 0.879 & 0.869 & 0.868 & 0.866 & 0.781 & 0.775 & 0.768 & 0.759 & 0.610 \\
\hline $\mathbf{2 5}$ & 0.939 & 0.939 & 0.937 & 0.935 & 0.934 & 0.934 & 0.930 & 0.929 & 0.928 & 0.927 & 0.862 \\
\hline $\mathbf{2 6}$ & 0.946 & 0.945 & 0.942 & 0.940 & 0.939 & 0.939 & 0.932 & 0.931 & 0.929 & 0.927 & 0.892 \\
\hline $\mathbf{2 7}$ & 0.851 & 0.848 & 0.832 & 0.822 & 0.818 & 0.815 & 0.779 & 0.773 & 0.765 & 0.756 & 0.626 \\
\hline $\mathbf{2 8}$ & 0.892 & 0.891 & 0.889 & 0.887 & 0.886 & 0.885 & 0.882 & 0.880 & 0.878 & 0.876 & 0.862 \\
\hline $\mathbf{2 9}$ & 0.919 & 0.919 & 0.918 & 0.917 & 0.916 & 0.915 & 0.914 & 0.913 & 0.912 & 0.911 & 0.903 \\
\hline
\end{tabular}


Table 5-7 Load Bus VCPI for Case 2

\begin{tabular}{|c|c|c|c|c|c|c|c|c|c|c|}
\hline & 1 & 1.05 & 1.1 & 1.15 & 1.2 & 1.25 & 1.3 & 1.35 & 1.4 & 1.45 \\
\hline 3 & 0.163 & 0.163 & 0.164 & 0.164 & 0.165 & 0.165 & 0.166 & 0.167 & 0.167 & 0.168 \\
\hline 4 & 0.258 & 0.258 & 0.262 & 0.264 & 0.267 & 0.269 & 0.271 & 0.274 & 0.274 & 0.279 \\
\hline 7 & 0.076 & 0.076 & 0.077 & 0.078 & 0.079 & 0.079 & 0.080 & 0.081 & 0.081 & 0.082 \\
\hline 8 & 0.164 & 0.164 & 0.167 & 0.168 & 0.169 & 0.171 & 0.172 & 0.174 & 0.174 & 0.177 \\
\hline 12 & 0.134 & 0.134 & 0.136 & 0.137 & 0.138 & 0.138 & 0.139 & 0.140 & 0.140 & 0.142 \\
\hline 15 & 0.220 & 0.220 & 0.224 & 0.226 & 0.228 & 0.231 & 0.233 & 0.235 & 0.235 & 0.240 \\
\hline 16 & 0.061 & 0.061 & 0.061 & 0.061 & 0.062 & 0.062 & 0.062 & 0.063 & 0.063 & 0.063 \\
\hline 18 & 0.076 & 0.076 & 0.077 & 0.077 & 0.078 & 0.078 & 0.079 & 0.079 & 0.079 & 0.081 \\
\hline 20 & 0.662 & 0.662 & 0.671 & 0.676 & 0.681 & 0.686 & 0.691 & 0.698 & 0.698 & 0.712 \\
\hline 21 & 0.186 & 0.186 & 0.189 & 0.191 & 0.192 & 0.194 & 0.195 & 0.197 & 0.197 & 0.200 \\
\hline 23 & 0.137 & 0.137 & 0.139 & 0.140 & 0.140 & 0.141 & 0.142 & 0.143 & 0.143 & 0.144 \\
\hline 24 & 0.155 & 0.155 & 0.155 & 0.155 & 0.156 & 0.156 & 0.156 & 0.156 & 0.156 & 0.157 \\
\hline 25 & 0.141 & 0.141 & 0.140 & 0.140 & 0.140 & 0.139 & 0.139 & 0.139 & 0.139 & 0.138 \\
\hline 26 & 0.125 & 0.125 & 0.124 & 0.124 & 0.123 & 0.123 & 0.122 & 0.122 & 0.122 & 0.121 \\
\hline 27 & 0.211 & 0.211 & 0.214 & 0.216 & 0.217 & 0.219 & 0.220 & 0.222 & 0.222 & 0.226 \\
\hline 28 & 0.217 & 0.217 & 0.218 & 0.218 & 0.219 & 0.219 & 0.220 & 0.220 & 0.220 & 0.222 \\
\hline 29 & 0.218 & 0.218 & 0.216 & 0.215 & 0.214 & 0.213 & 0.213 & 0.212 & 0.212 & 0.210 \\
\hline
\end{tabular}

Table 5-8 Load Bus VCPI for Case 2 (cont.)

\begin{tabular}{|c|c|c|c|c|c|c|c|c|c|c|c|}
\hline \# & 1.5 & 1.55 & 1.6 & 1.65 & 1.7 & 1.75 & 1.8 & 1.85 & 1.9 & 1.95 & 1.98 \\
\hline 3 & 0.169 & 0.170 & 0.171 & 0.173 & 0.175 & 0.177 & 0.180 & 0.184 & 0.188 & 0.193 & 0.201 \\
\hline 4 & 0.282 & 0.285 & 0.289 & 0.293 & 0.298 & 0.303 & 0.311 & 0.319 & 0.329 & 0.341 & 0.357 \\
\hline 7 & 0.083 & 0.084 & 0.085 & 0.086 & 0.087 & 0.088 & 0.090 & 0.093 & 0.095 & 0.098 & 0.102 \\
\hline 8 & 0.179 & 0.180 & 0.182 & 0.185 & 0.188 & 0.190 & 0.195 & 0.199 & 0.204 & 0.210 & 0.219 \\
\hline 12 & 0.143 & 0.144 & 0.146 & 0.148 & 0.151 & 0.154 & 0.158 & 0.163 & 0.169 & 0.176 & 0.186 \\
\hline 15 & 0.243 & 0.246 & 0.250 & 0.255 & 0.260 & 0.265 & 0.275 & 0.286 & 0.299 & 0.315 & 0.338 \\
\hline 16 & 0.064 & 0.064 & 0.065 & 0.066 & 0.067 & 0.068 & 0.070 & 0.073 & 0.076 & 0.080 & 0.086 \\
\hline 18 & 0.081 & 0.082 & 0.083 & 0.084 & 0.085 & 0.086 & 0.089 & 0.091 & 0.094 & 0.098 & 0.103 \\
\hline 20 & 0.719 & 0.726 & 0.738 & 0.753 & 0.769 & 0.786 & 0.814 & 0.849 & 0.889 & 0.940 & 1.010 \\
\hline 21 & 0.202 & 0.204 & 0.207 & 0.211 & 0.215 & 0.219 & 0.228 & 0.239 & 0.251 & 0.267 & 0.289 \\
\hline 23 & 0.145 & 0.147 & 0.148 & 0.150 & 0.152 & 0.155 & 0.161 & 0.168 & 0.177 & 0.188 & 0.203 \\
\hline 24 & 0.157 & 0.158 & 0.159 & 0.161 & 0.163 & 0.165 & 0.170 & 0.176 & 0.183 & 0.192 & 0.205 \\
\hline 25 & 0.138 & 0.138 & 0.138 & 0.138 & 0.138 & 0.138 & 0.138 & 0.139 & 0.140 & 0.140 & 0.143 \\
\hline 26 & 0.121 & 0.120 & 0.120 & 0.120 & 0.120 & 0.120 & 0.120 & 0.120 & 0.121 & 0.122 & 0.124 \\
\hline 27 & 0.228 & 0.230 & 0.233 & 0.236 & 0.239 & 0.243 & 0.249 & 0.256 & 0.264 & 0.273 & 0.286 \\
\hline 28 & 0.222 & 0.223 & 0.224 & 0.226 & 0.227 & 0.228 & 0.231 & 0.233 & 0.236 & 0.239 & 0.243 \\
\hline 29 & 0.209 & 0.209 & 0.208 & 0.207 & 0.207 & 0.206 & 0.206 & 0.206 & 0.206 & 0.206 & 0.207 \\
\hline
\end{tabular}


Once again, it is shown that both cases approach their respective limits. The VCPI for bus 20 actually exceeds its theoretical index limit of one when the system collapses. This suggests that a VCPI exceeding a value of 1 can also mean the system has collapsed. With further investigation, a margin can be estimated to when the VSI or VCPI can indicate the system is approaching instability.

\subsubsection{Voltage Collapse Predictability From Indices}

As stated previously, an index should be able to represent the state of the system and results can be understood at an intuitive level. It is therefore useful to see whether an index value can detect the proximity to voltage collapse without knowing the nature of the load. To demonstrate this, consider case 1 where real power is increased at constant power factor, and case 2 where only the reactive power is increased. An additional point for each index was calculated by the closest value to collapse without diverging, within .001 times the final load factor. The results are tabulated in table 5-9. 
Table 5-9 Indices before collapse: Case 1 and 2

\begin{tabular}{|c|c|c|c|c|}
\hline & VSI & & VCPI & \\
\hline & Case 1 & Case 2 & Case 1 & Case 2 \\
\hline Bus No & 1.265 & 1.98 & 1.265 & 1.98 \\
\hline 3 & 0.621 & 0.686 & 0.231 & 0.201 \\
\hline 4 & 0.179 & 0.140 & 0.415 & 0.357 \\
\hline 7 & 0.402 & 0.412 & 0.127 & 0.102 \\
\hline 8 & 0.087 & 0.081 & 0.273 & 0.219 \\
\hline 12 & 0.613 & 0.399 & 0.262 & 0.186 \\
\hline 15 & 0.372 & 0.217 & 0.329 & 0.338 \\
\hline 16 & 0.524 & 0.531 & 0.085 & 0.086 \\
\hline 18 & 0.765 & 0.756 & 0.108 & 0.103 \\
\hline 20 & 0.052 & 0.071 & 0.836 & 1.010 \\
\hline 21 & 0.441 & 0.283 & 0.260 & 0.289 \\
\hline 23 & 0.475 & 0.355 & 0.185 & 0.203 \\
\hline 24 & 0.538 & 0.610 & 0.215 & 0.205 \\
\hline 25 & 0.859 & 0.862 & 0.178 & 0.143 \\
\hline 26 & 0.888 & 0.892 & 0.149 & 0.124 \\
\hline 27 & 0.659 & 0.626 & 0.293 & 0.286 \\
\hline 28 & 0.854 & 0.862 & 0.283 & 0.243 \\
\hline 29 & 0.892 & 0.903 & 0.259 & 0.207 \\
\hline
\end{tabular}

When comparing the last values before the voltage collapse, it is quite clear that most indices approach similar values, showing that the indices are independent of the nature of the load near voltage collapse. Since these results are similar, a comment on the differences should be made. First of all, a bus displaying an index difference greater than .15 between the two cases is highlighted in red. Both VSI and VCPI, give bus 12 significantly different values near collapse. There are also notable differences for buses 15 and 21 for the VSI and bus 20 for the VCPI. 
An alternative way to view the load independence would be to plot each index against its apparent power margin, which is computed as the difference between the apparent power when the system collapses and the current apparent power. Consider the indices for bus 20 as plotted on figures 5.12 and 5.13. As the apparent power margin decreases, the VCPI converges towards similar values.

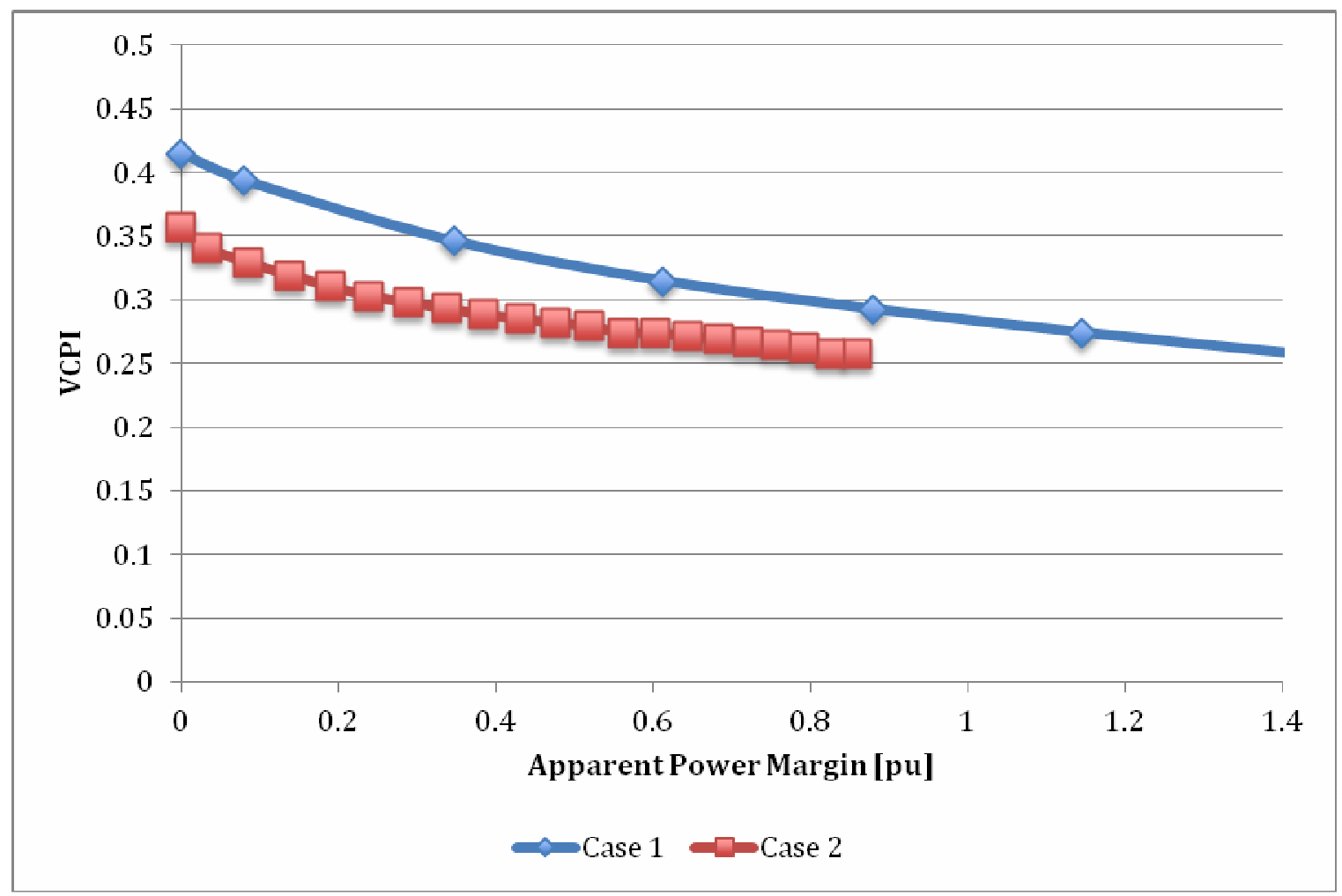

Figure 5.12 VCPI vs. Apparent Power Margin for Case 1 and 2 


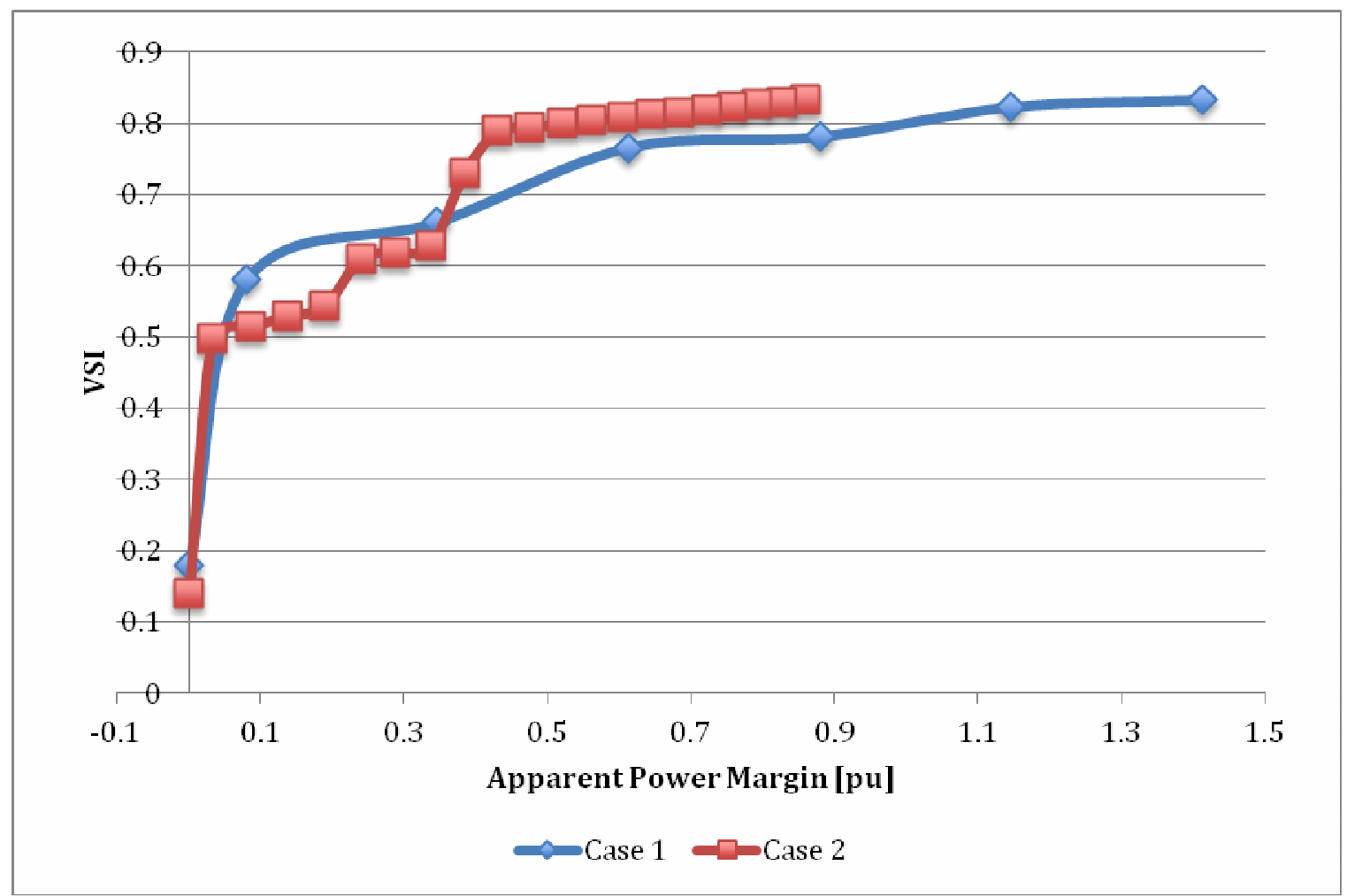

Figure 5.13 VSI vs. Apparent Power Margin for Case 1 and 2

From these comparisons, the indices are capable of providing information on how far the system is to the voltage collapse regardless of the characteristic of the load. This can be understood as how the index itself can give insight without knowing any other parameters of the system.

\subsection{Case 3: IEEE 39 Bus System -Loss of transmission line}

Similar to the first case, the loads in the 39-bus system were subject to real power increase at constant power factor. Each load was increased by $2.5 \%$ of the initial base case until the system collapsed. However, on the sixth load increase, a transmission line was removed to simulate the loss of a heavily loaded line. The line chosen was based on the limit of the line; the line that had the most amount of apparent power flowing with 
respect to maximum ampacity, was removed. After each load increase, the indices were computed as prescribed by the algorithms according to figure 5.1 and 5.2. Initially the system is running under normal operating conditions. Each load is increased as previously mentioned. On the sixth load step, line 10-13 is tripped having been loaded $82 \%$ of its $600 \mathrm{MVA}$ line rating.

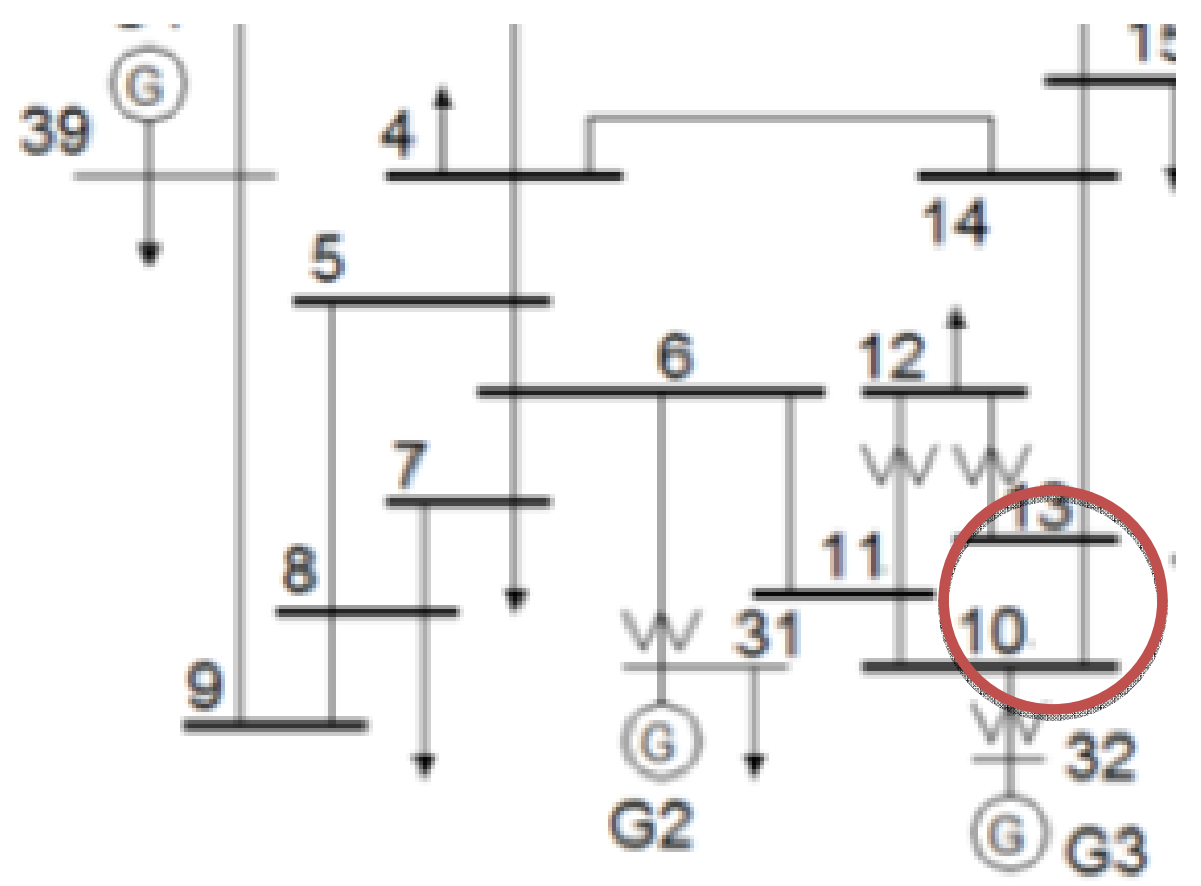

Figure 5.14 Line 10-13 Trip 
Table 5-10 shows the system VSI before and after the line has tripped.

Table 5-10 Before and After Line Tripped

\begin{tabular}{|r|c|c|c|c|c|c|}
\hline & \multicolumn{3}{|c|}{ VSI } & \multicolumn{3}{c|}{ VCPI } \\
\hline Bus No. & Before & After & $\% \Delta$ & Before & After & $\% \Delta$ \\
\hline 3 & 0.899 & 0.898 & -0.157 & 0.192 & 0.195 & 1.435 \\
\hline 4 & 0.690 & 0.682 & -1.194 & 0.315 & 0.325 & 3.109 \\
\hline 7 & 0.799 & 0.780 & -2.350 & 0.094 & 0.096 & 2.770 \\
\hline 8 & 0.612 & 0.581 & -5.023 & 0.203 & 0.208 & 2.750 \\
\hline 12 & 0.822 & 0.815 & -0.865 & 0.177 & 0.188 & 5.973 \\
\hline 15 & 0.819 & 0.814 & -0.654 & 0.264 & 0.269 & 1.803 \\
\hline 16 & 0.921 & 0.920 & -0.137 & 0.071 & 0.072 & 1.047 \\
\hline 18 & 0.934 & 0.934 & -0.089 & 0.090 & 0.091 & 1.244 \\
\hline 20 & 0.816 & 0.815 & -0.052 & 0.740 & 0.742 & 0.170 \\
\hline 21 & 0.881 & 0.880 & -0.120 & 0.220 & 0.221 & 0.760 \\
\hline 23 & 0.924 & 0.924 & -0.038 & 0.160 & 0.161 & 0.411 \\
\hline 24 & 0.907 & 0.906 & -0.130 & 0.181 & 0.183 & 0.939 \\
\hline 25 & 0.936 & 0.936 & -0.038 & 0.160 & 0.160 & 0.465 \\
\hline 26 & 0.942 & 0.942 & -0.043 & 0.136 & 0.137 & 0.393 \\
\hline 27 & 0.851 & 0.850 & -0.159 & 0.249 & 0.252 & 0.906 \\
\hline 28 & 0.885 & 0.885 & -0.045 & 0.251 & 0.252 & 0.343 \\
\hline 29 & 0.912 & 0.912 & -0.023 & 0.240 & 0.240 & 0.163 \\
\hline
\end{tabular}

From table 5-10 both indices agree that the removal of the line has the greatest impact on buses 4,7, and 8. As denoted in the darker shade of red, the VSI shows the most significant change on bus 8 , while the VCPI shows the most significant change on bus 12. By viewing the most affected bus locations with respect to the line outage, it is seen that the affected buses are within physical distance from it. Attention is then directed to this area as it can reveal sensitive bus locations that can result in voltage collapse. Buses 4,7,8 and 12 and global indices are plotted in figures 5.15 and 5.16 for each load increase. 


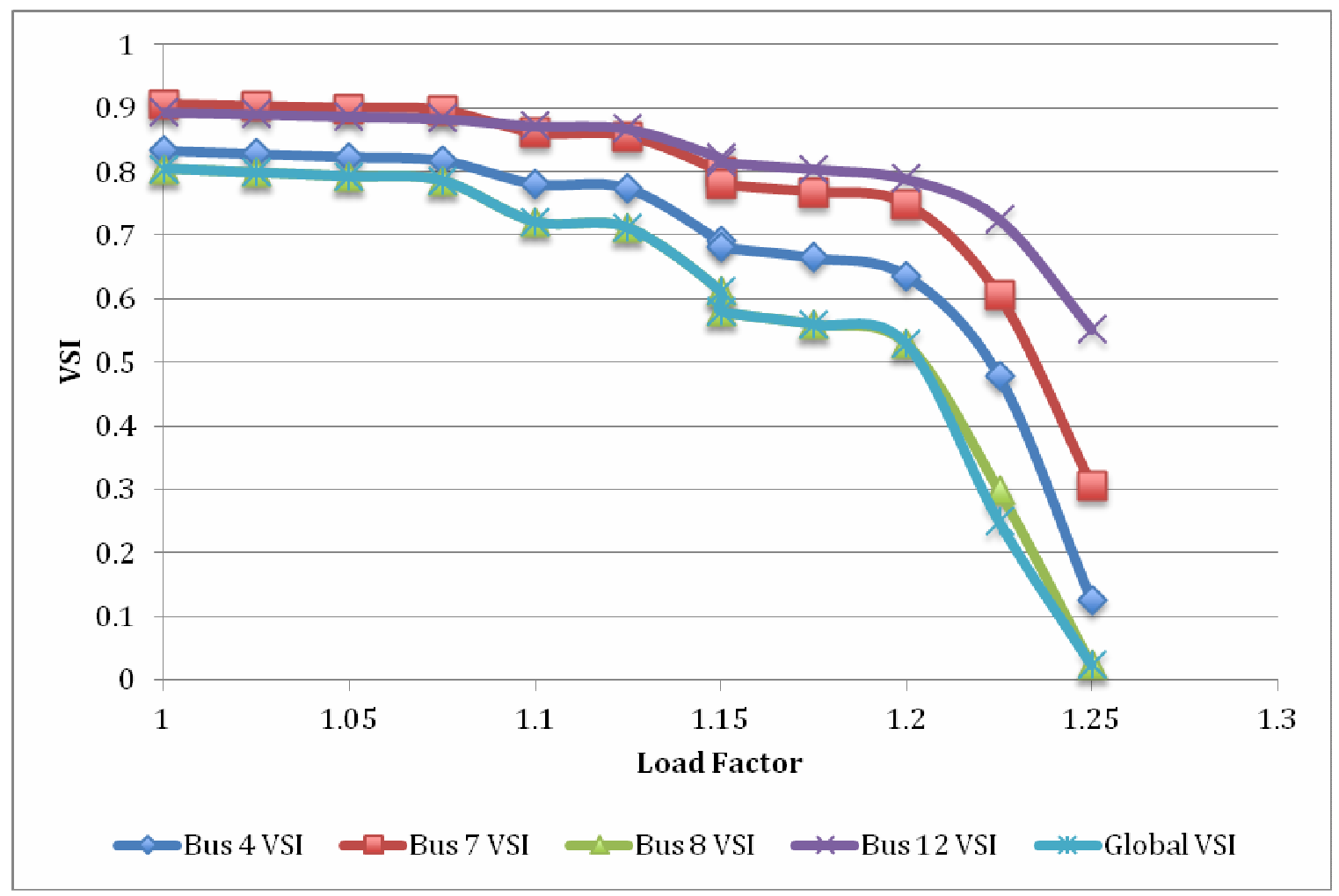

Figure 5.15 VSIs for Case 3: Bus 4, 6, 8, 12 and Global

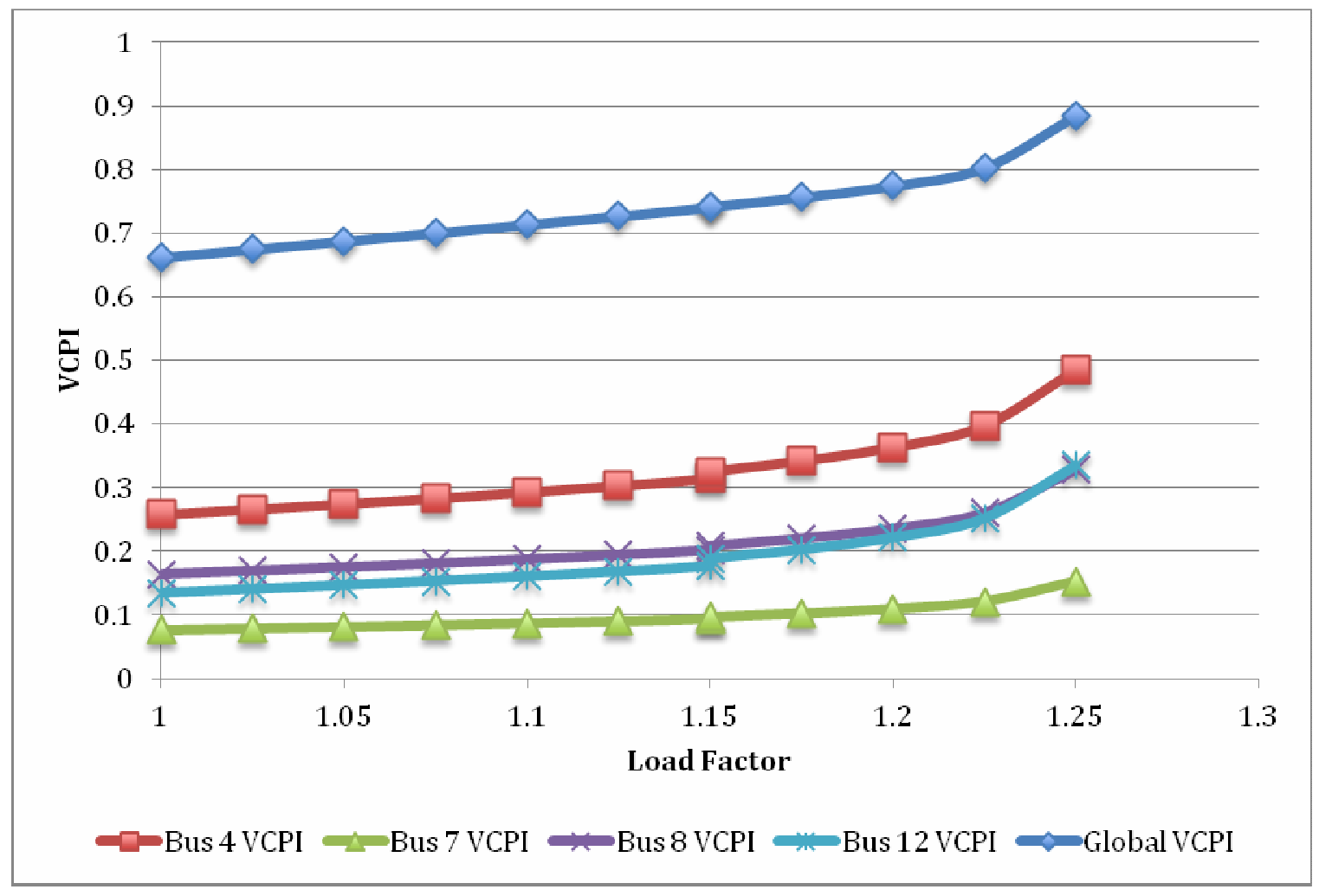

Figure 5.16 VCPIs for Case 3: Bus 4, 6, 8, 12 and Global 
The VSIs according to figure 5.15 reveal that bus 8 approaches instability limits quicker before the surrounding buses. On the other hand, the VCPIs according to figure 5.16 illustrate that another bus reaches instability limits before any of the other surrounding buses are even close to instability limits. By looking at table 5-11, the last values before and after collapse, the buses in the area of the line outage are less stable than compared to those that are not. Since both indices are dependent on system topology, results show the instable area has a negative impact on the rest of the system, and upon a major load increase can cause a system collapse. Even though the studied load bus VCPIs do not come close to the ideal marginal limit, they are still of higher magnitude than the other load buses with the exception of bus 7 .

Table 5-11 Load Bus Indices for Case 3, before and after collapse

\begin{tabular}{|r|l|l|l|l|}
\hline & \multicolumn{2}{|c|}{ VSI } & \multicolumn{2}{c|}{ VCPI } \\
\hline \multicolumn{1}{r|}{ Fus } & 1.225 & 1.25 & 1.225 & 1.25 \\
\hline 3 & 0.840 & 0.601 & 0.222 & 0.250 \\
\hline $\mathbf{4}$ & $\mathbf{0 . 4 7 9}$ & $\mathbf{0 . 1 2 6}$ & $\mathbf{0 . 3 9 7}$ & $\mathbf{0 . 4 8 5}$ \\
\hline $\mathbf{7}$ & $\mathbf{0 . 6 0 5}$ & $\mathbf{0 . 3 0 6}$ & $\mathbf{0 . 1 2 1}$ & $\mathbf{0 . 1 5 3}$ \\
\hline $\mathbf{8}$ & $\mathbf{0 . 2 9 9}$ & $\mathbf{0 . 0 2 4}$ & $\mathbf{0 . 2 6 0}$ & $\mathbf{0 . 3 2 8}$ \\
\hline $\mathbf{1 2}$ & $\mathbf{0 . 7 2 6}$ & $\mathbf{0 . 5 5 1}$ & $\mathbf{0 . 2 5 2}$ & $\mathbf{0 . 3 3 5}$ \\
\hline $\mathbf{1 5}$ & $\mathbf{0 . 6 7 4}$ & $\mathbf{0 . 3 4 4}$ & $\mathbf{0 . 3 1 2}$ & $\mathbf{0 . 3 6 1}$ \\
\hline 16 & 0.813 & 0.505 & 0.081 & 0.092 \\
\hline 18 & 0.895 & 0.754 & 0.103 & 0.116 \\
\hline 20 & 0.250 & 0.047 & 0.802 & 0.884 \\
\hline 21 & 0.760 & 0.428 & 0.248 & 0.277 \\
\hline 23 & 0.853 & 0.470 & 0.177 & 0.192 \\
\hline 24 & 0.817 & 0.519 & 0.205 & 0.229 \\
\hline 25 & 0.924 & 0.859 & 0.173 & 0.183 \\
\hline 26 & 0.929 & 0.887 & 0.145 & 0.153 \\
\hline 27 & 0.800 & 0.652 & 0.282 & 0.309 \\
\hline 28 & 0.872 & 0.856 & 0.274 & 0.287 \\
\hline 29 & 0.903 & 0.893 & 0.253 & 0.261 \\
\hline & 0.250 & 0.024 & 0.802 & 0.884 \\
\hline & & & & \\
\hline Global & & & & \\
\hline
\end{tabular}


These results can be verified by examining the load flow solution. Table 5-12 shows the power flows and losses associated for each line. The branches with the most significant increases in losses are only displayed. The full results for each branch can be found in appendix B. Once the line was tripped, the power through line 10-13 has now been routed onto other lines. The most significant increase is shown through line 4-5. An additional $300 \mathrm{MW}$ is transferred across this line and consequently increasing the reactive losses by over 50 MVARs. Likewise, the branches this area suffer similar fates as a result of this line loss.

Table 5-12 Power Flow and Line Losses: Before and After Line Trip

\begin{tabular}{|c|c|c|c|c|c|c|c|c|c|}
\hline \multicolumn{2}{|c|}{ Line } & \multicolumn{6}{c|}{ Before } & \multicolumn{4}{c|}{ After } \\
\hline FROM & TO & $\begin{array}{c}\text { Line } \\
\text { MW }\end{array}$ & $\begin{array}{c}\text { Line } \\
\text { MVAR }\end{array}$ & $\begin{array}{c}\text { Line } \\
\text { MVA }\end{array}$ & $\begin{array}{c}\text { Loss } \\
\text { MVAR }\end{array}$ & $\begin{array}{c}\text { Line } \\
\text { MW }\end{array}$ & $\begin{array}{c}\text { Line } \\
\text { MVAR }\end{array}$ & $\begin{array}{c}\text { Line } \\
\text { MVA }\end{array}$ & $\begin{array}{c}\text { LineLoss } \\
\text { MVAR }\end{array}$ \\
\hline 10 & 13 & 490.6 & 49 & 493 & 3.49 & 0 & 0 & 0 & 0 \\
\hline 3 & 18 & 88.2 & -25.2 & 91.7 & -21.04 & 130.3 & -35.8 & 135.1 & -19.47 \\
\hline 3 & 4 & -130.2 & 191.9 & 232 & -9.89 & -152.4 & 227.9 & 274.2 & -4.61 \\
\hline 4 & 14 & -236.1 & -65.2 & 245 & -5.24 & 35 & -72.9 & 80.9 & -12.07 \\
\hline 4 & 5 & -469.9 & 55.5 & 473.1 & 17.52 & -763.4 & 93.8 & 769.2 & 69.93 \\
\hline 5 & 8 & 374.5 & 61.3 & 379.4 & 3.33 & 365.2 & 52 & 368.9 & 3.23 \\
\hline 5 & 6 & -846.2 & -23.3 & 846.5 & 15.58 & -1133.7 & -28.1 & 1134.1 & 32.37 \\
\hline 6 & 31 & -1261 & -27.9 & 1261.3 & 478.82 & -1267.8 & -61.9 & 1269.3 & 497.9 \\
\hline 6 & 11 & -138.5 & -112 & 178.1 & -10.73 & -467.2 & -99.5 & 477.7 & 7.07 \\
\hline 6 & 7 & 551.8 & 101 & 560.9 & 19.93 & 598.4 & 100.9 & 606.9 & 26.36 \\
\hline 7 & 8 & 280.9 & -15.5 & 281.3 & -3.31 & 327.2 & -22.1 & 327.9 & -1.57 \\
\hline 8 & 9 & 53.5 & -156.6 & 165.5 & -28.49 & 90.4 & -174.1 & 196.2 & -23.3 \\
\hline 9 & 39 & 53 & -128.2 & 138.7 & -123.6 & 89.5 & -150.8 & 175.4 & -120.71 \\
\hline
\end{tabular}

As seen from the power flow solution, removing a line changes the network topology resulting in an alternative power flow condition. Since both real power load and generation remain constant, the total real power transmitted across the lines remains constant assuming there are no additional losses. In this case however, removing a line 
increases the total line losses in the network. Since the generation must meet the load demand, the line losses are accounted for by a change in slack bus generation. The generation and loads are before and after the line trip are displayed in table 5-13. The line losses are displayed in table 5-14.

Table 5-13 Generation and Loads before and after Line Trip

\begin{tabular}{|c|c|c|c|c|c|c|c|}
\hline \multicolumn{4}{|c|}{ Before } & \multicolumn{4}{|c|}{ After } \\
\hline \multicolumn{2}{|c|}{ Generation } & \multicolumn{2}{|c|}{ Load } & \multicolumn{2}{|c|}{ Generation } & \multicolumn{2}{|c|}{ Load } \\
\hline Bus No. & MW & Bus No. & MW & Bus No. & MW & Bus No. & MW \\
\hline 30 & 250 & 3 & 370.3 & 30 & 250 & 3 & 370.3 \\
\hline 31 & 1270.21 & 4 & 575 & 31 & 1276.97 & 4 & 575 \\
\hline 32 & 650 & 7 & 268.87 & 32 & 650 & 7 & 268.87 \\
\hline 33 & 632 & 8 & 600.3 & 33 & 632 & 8 & 600.3 \\
\hline 34 & 508 & 12 & 8.62 & 34 & 508 & 12 & 8.62 \\
\hline 35 & 650 & 15 & 368 & 35 & 650 & 15 & 368 \\
\hline 36 & 560 & 16 & 378.35 & 36 & 560 & 16 & 378.35 \\
\hline 37 & 540 & 18 & 181.7 & 37 & 540 & 18 & 181.7 \\
\hline 38 & 830 & 20 & 722.2 & 38 & 830 & 20 & 722.2 \\
\hline \multirow[t]{10}{*}{39} & 1000 & 21 & 315.1 & 39 & 1000 & 21 & 315.1 \\
\hline & & 23 & 284.62 & & & 23 & 284.62 \\
\hline & & 24 & 354.89 & & & 24 & 354.89 \\
\hline & & 25 & 257.6 & & & 25 & 257.6 \\
\hline & & 26 & 159.85 & & & 26 & 159.85 \\
\hline & & 27 & 323.15 & & & 27 & 323.15 \\
\hline & & 28 & 236.9 & & & 28 & 236.9 \\
\hline & & 29 & 326.03 & & & 29 & 326.03 \\
\hline & & 31 & 9.2 & & & 31 & 9.2 \\
\hline & & 39 & 1104 & & & 39 & 1104 \\
\hline Total & 6890.21 & & 6844.68 & & 6896.97 & & 6844.68 \\
\hline \multicolumn{2}{|l|}{$\Delta \mathrm{P}$} & & 45.53 & & & & 52.29 \\
\hline
\end{tabular}

Where: $\quad \Delta P=P_{g e n}-P_{\text {load }}$

Ex:

$$
\Delta P_{\text {before }}=6890.21-6844.68=45.53 \mathrm{MW}
$$


Table 5-14 Line Losses Before and After Line Trip

\begin{tabular}{|c|c|c|c|}
\hline \multicolumn{2}{|c|}{ Branch } & \multicolumn{2}{|c|}{ Line Loss [MW] } \\
\hline From & To & Before & After \\
\hline 1 & 2 & 0.09 & 0.01 \\
\hline 1 & 39 & 0.07 & 0.04 \\
\hline 10 & 13 & 0.99 & 0 \\
\hline 10 & 32 & 0 & 0 \\
\hline 10 & 11 & 0.19 & 1.9 \\
\hline 12 & 11 & 0.04 & 0.63 \\
\hline 12 & 13 & 0.05 & 0.56 \\
\hline 13 & 14 & 2.32 & 0.31 \\
\hline 14 & 15 & 1.35 & 0.96 \\
\hline 15 & 16 & 0.57 & 0.85 \\
\hline 16 & 19 & 2.66 & 2.73 \\
\hline 16 & 17 & 0.2 & 0.09 \\
\hline 16 & 24 & 0.05 & 0.05 \\
\hline 16 & 21 & 0.57 & 0.58 \\
\hline 17 & 27 & 0.09 & 0.07 \\
\hline 17 & 18 & 0.07 & 0.03 \\
\hline 19 & 20 & 0.35 & 0.35 \\
\hline 19 & 33 & 3.01 & 3.04 \\
\hline 2 & 30 & 0 & 0 \\
\hline 2 & 3 & 1.67 & 1.97 \\
\hline 2 & 25 & 1.29 & 0.96 \\
\hline 20 & 34 & 2.55 & 2.57 \\
\hline 21 & 22 & 2.76 & 2.8 \\
\hline 22 & 35 & 0 & 0 \\
\hline 22 & 23 & 0.04 & 0.04 \\
\hline 23 & 36 & 1.46 & 1.47 \\
\hline 23 & 24 & 2.28 & 2.31 \\
\hline 25 & 37 & 1.67 & 1.67 \\
\hline 25 & 26 & 0.64 & 0.8 \\
\hline 26 & 29 & 1.32 & 1.32 \\
\hline 26 & 28 & 0.41 & 0.41 \\
\hline 26 & 27 & 0.92 & 1.05 \\
\hline 28 & 29 & 1.48 & 1.48 \\
\hline 29 & 38 & 5.25 & 5.26 \\
\hline 3 & 18 & 0.09 & 0.19 \\
\hline 3 & 4 & 0.74 & 1.03 \\
\hline 4 & 14 & 0.5 & 0.05 \\
\hline 4 & 5 & 1.89 & 5.14 \\
\hline 5 & 8 & 1.23 & 1.19 \\
\hline 5 & 6 & 1.51 & 2.8 \\
\hline 6 & 31 & 0 & 0 \\
\hline 6 & 11 & 0.22 & 1.72 \\
\hline 6 & 7 & 1.99 & 2.39 \\
\hline 7 & 8 & 0.34 & 0.48 \\
\hline 8 & 9 & 0.55 & 0.84 \\
\hline 9 & 39 & 0.07 & 0.16 \\
\hline
\end{tabular}




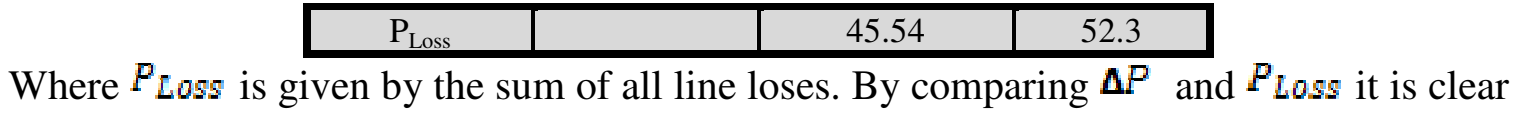
that $\boldsymbol{\Delta P}$ is approximately equal to $P_{\text {Lass }}$. Thus, it is seen that the power flows across the lines remain constant when the system topology changes. As a result the line losses also change. Therefore,

$$
P_{\text {gen }}=P_{\text {load }}+P_{\text {loss }}=P_{\text {Flow }}+P_{\text {loss }}
$$

Where $P_{\text {Flow }}$ is the net power through all the lines.

In summary, it is shown that even when a line is removed, the two indices will respond accordingly. Therefore, upon a line tripping and by monitoring significant changes in index values, certain areas should be monitored more closely as they are more prone to voltage collapse.

\subsection{Case 4: IEEE 39 Bus System - Intermittent Generation}

This case will look at a particular area as shown in figure 5.17. This area contains two generators serving 4 local loads while connected to the rest of the 39-Bus system.

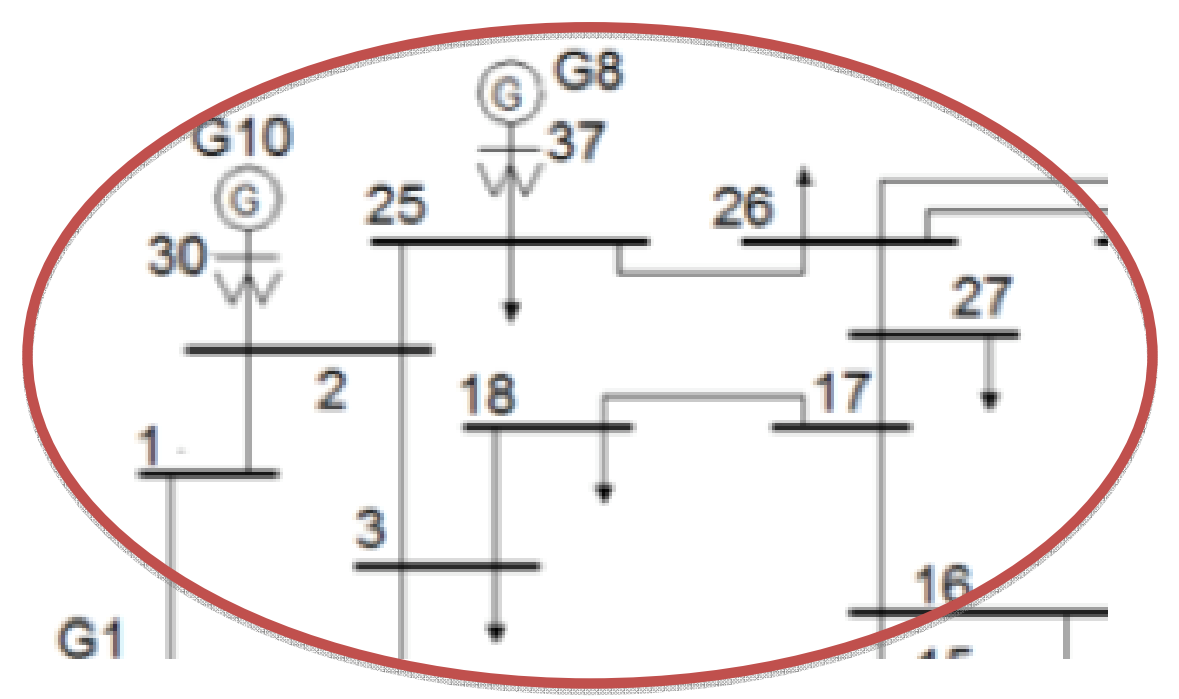

Figure 5.17 Case 4 Area of Interest 
Bus 18 will be loaded to $66 \%$ (at constant power factor) of its maximum load capacity in order to simulate a loaded situation. An offline study was done to verify the maximum load bus 18 could handle before collapsing. This value was $1317 \mathrm{MW}$ at a power factor of 0.98 . The load for bus 18 at $66 \%$ of its maximum capacity was $869 \mathrm{MW}$ and 165 MVAR. The generation at bus 30 was varied for different levels to represent different levels of wind. The amount of wind generation will be randomly varied from 0 to 550 MW for a period of 24 hours, measurements were take every one hour.

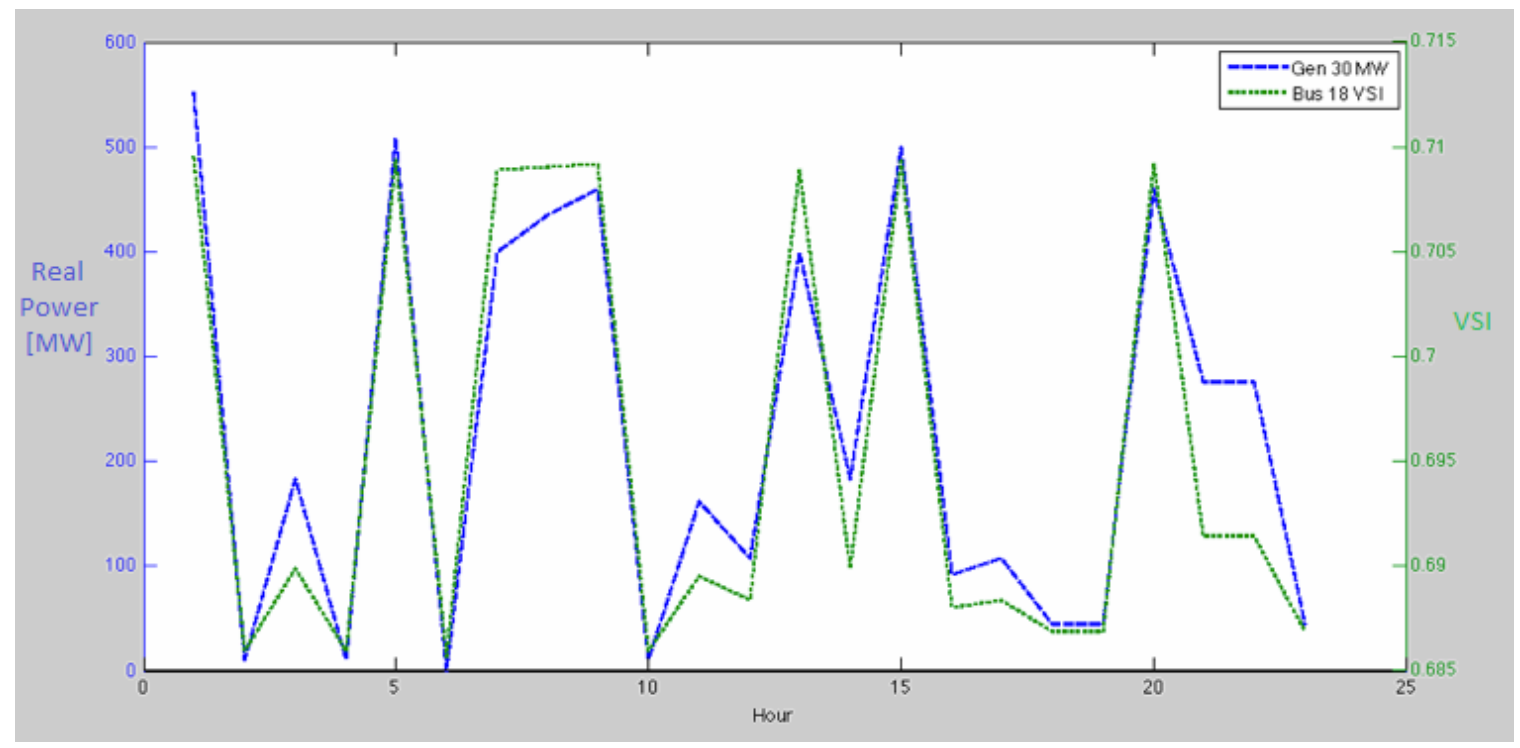

Figure 5.18 Bus 18 VSI for various Wind Generation output 


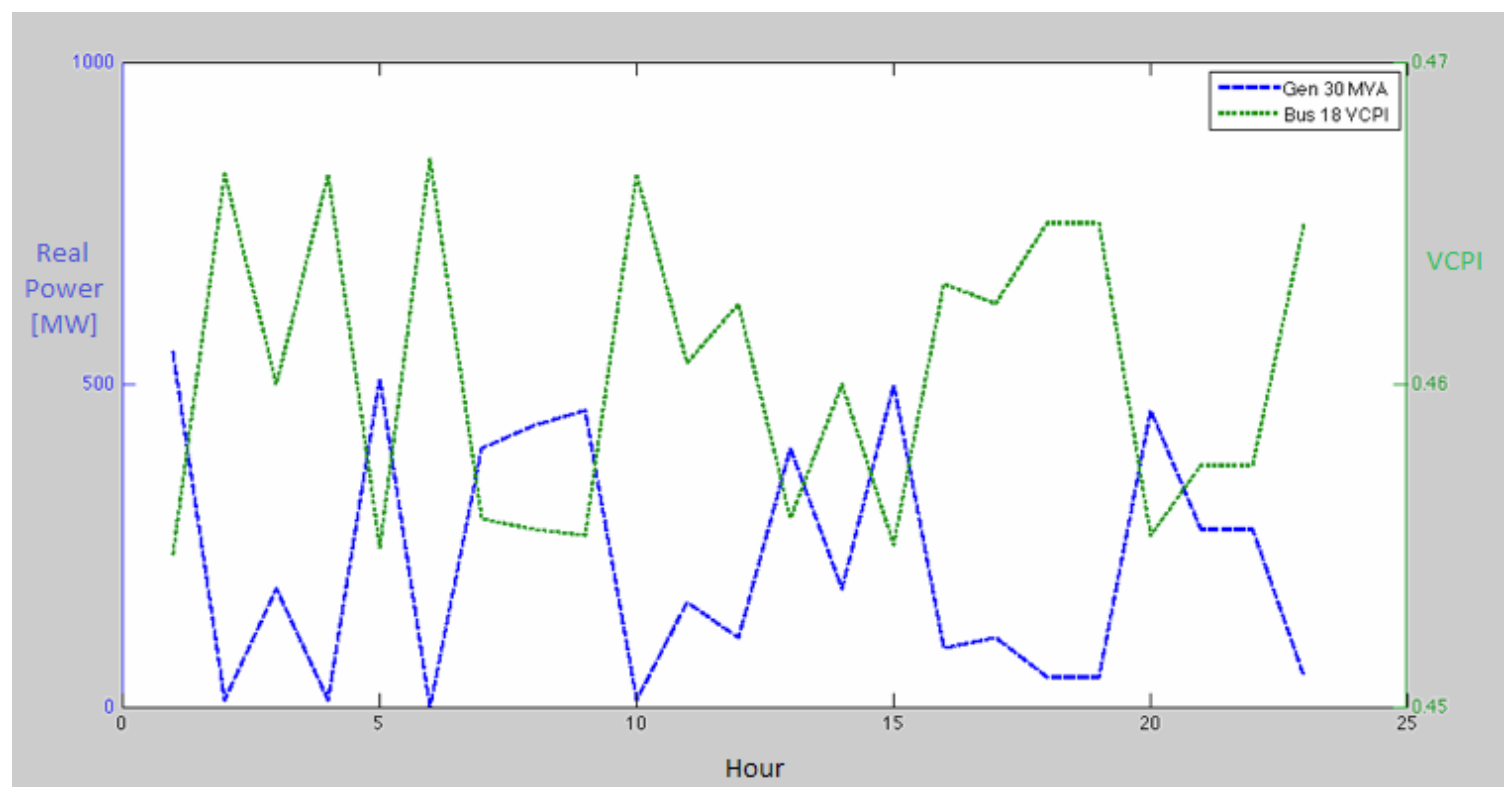

Figure 5.19 Bus 18 VCPI for various Wind Generation output

From figures 5.18 and 5.19, the index at bus 18 varies as the wind generation output varies. The calculated index is for bus 18 for both cases, to enable easy comparison. It can be seen that the value of VSI is higher for wind penetration levels equal to or higher than 300MW. Therefore regardless of the situation, each index will show variations with respect to its current state. Results also reveal that for an area where the power is delivered by an intermittent source, the indices are still within stable limits. When the wind loading is high, indices show that the area is even more stable. Although in this case the indices do not show any signs of instability, they serve as an adequate monitoring tool, especially for unpredictable conditions.

\subsection{Stability Margin}

It is shown that the indices can detect the instability limits in both test systems. However, in most cases the VSI and VCPI approach 0 and 1 respectively when the system is on the verge of collapse. In order for the indices to determine the state of the 
system, a margin should be made which distinguishes, when the system is near a collapse. By analyzing the global index at the last load increase before collapse for case 1 and case 2, the VSI provides an index of 0.262 and .163 respectively. Likewise, the VCPI provides an index of .812 and .94 respectively. Recalling the fact that a system wide collapse can occur within seconds, the margin should be established to give ample time to analyze and react to the situation. For both indices, a 0.3 index margin should be appropriate.

\subsection{Weakest Bus}

In traditional studies it is often useful to consider ranking the strength of each load bus. As previously mentioned, these indices can be used to determine the strength of each bus depending on the value of each index. The indices presented in chapter 3 , propose methods that show their ability to determine weak branches and or buses. However, the analyzed indices in this chapter do not present any method to determine the weak buses. An attempt to determine the weak bus is initiated first by considering indices at the first load increase, and the last increase before collapsing.

Table 5-15 and Table 5-16 show the rankings for a given analysis for the base case and $6^{\text {th }}$ load increase. In addition, well known computations, QV and modal analysis were performed on all load buses in order to give an adequate comparison. Full calculations for QV and modal analysis are shown appendix C and D respectively. 
Table 5-15 Comparison Of Rankings Between Various Methods: First Load Increase

\begin{tabular}{|c|c|c|c|c|c|c|c|c|}
\hline & \multicolumn{2}{|c|}{ VSI } & \multicolumn{2}{c|}{ VCPI } & \multicolumn{2}{c|}{ MODAL } & \multicolumn{2}{c|}{ QV } \\
\hline Ranking & Bus No & Index & Bus No & Index & Bus No & Participation & $\begin{array}{c}\text { Bus } \\
\text { No }\end{array}$ & Margin \\
\hline 1 & 8 & 0.792 & 20 & 0.662 & 12 & 0.1085 & 20 & -460.83 \\
\hline 2 & 4 & 0.823 & 4 & 0.258 & 7 & 0.0669 & 29 & -490.19 \\
\hline 3 & 20 & 0.833 & 15 & 0.220 & 8 & 0.0646 & 28 & -512.83 \\
\hline 4 & 15 & 0.860 & 29 & 0.218 & 4 & 0.0631 & 23 & -559.8 \\
\hline 5 & 27 & 0.870 & 28 & 0.217 & 15 & 0.0441 & 12 & -595.89 \\
\hline 6 & 12 & 0.887 & 27 & 0.211 & 18 & 0.0379 & 21 & -633.1 \\
\hline 7 & 28 & 0.896 & 21 & 0.186 & 3 & 0.0331 & 24 & -745.75 \\
\hline 8 & 21 & 0.896 & 8 & 0.164 & 27 & 0.0311 & 16 & -811.01 \\
\hline 9 & 7 & 0.899 & 3 & 0.163 & 16 & 0.02792 & 15 & -811.21 \\
\hline 10 & 29 & 0.920 & 24 & 0.155 & 24 & 0.0261 & 26 & -890.19 \\
\hline 11 & 24 & 0.921 & 25 & 0.141 & 26 & 0.0195 & 27 & -917.6 \\
\hline 12 & 3 & 0.922 & 23 & 0.137 & 21 & 0.0177 & 18 & -986.68 \\
\hline 13 & 23 & 0.932 & 12 & 0.134 & 28 & 0.0086 & 8 & -1002.09 \\
\hline 14 & 16 & 0.935 & 26 & 0.125 & 25 & 0.0069 & 7 & -1008.73 \\
\hline 15 & 25 & 0.943 & 18 & 0.076 & 23 & 0.0063 & 4 & -1043.78 \\
\hline 16 & 18 & 0.946 & 7 & 0.076 & 29 & 0.0040 & 25 & -1074.78 \\
\hline 17 & 26 & 0.949 & 16 & 0.061 & 20 & 0.0017 & 3 & -1087.59 \\
\hline
\end{tabular}

For the base case it is quite clear that a majority of the values disagree over which bus is the critical bus. The only similar ranking is between the VSI and VCPI for bus 2, denoting that it is the second weakest load bus. In the previous analysis it had shown that for base case operating conditions, the system is a stable system. So far, there is insufficient information to make any concrete conclusion on any rankings. Next, consider the case when the load is near its collapse point, the sixth load increase. 
Table 5-16 Comparison Of Rankings Between Various Methods: Final Load Increase

\begin{tabular}{|c|c|c|c|c|c|c|c|c|}
\hline & VSI & & VCPI & & MODAL & & QV & \\
\hline Ranking & Bus No & Index & Bus No & Index & Bus No & $\begin{array}{l}\text { Participati } \\
\text { on }\end{array}$ & $\begin{array}{l}\text { Bus } \\
\text { No }\end{array}$ & Margin \\
\hline 1 & 20 & 0.262 & 20 & 0.812 & 12 & 0.1169 & 12 & -106.88 \\
\hline 2 & 8 & 0.497 & 4 & 0.394 & 7 & 0.0747 & 7 & -118.71 \\
\hline 3 & 4 & 0.580 & 15 & 0.315 & 8 & 0.0719 & 8 & -119.39 \\
\hline 4 & 15 & 0.708 & 27 & 0.286 & 4 & 0.0655 & 20 & -127.08 \\
\hline 5 & 7 & 0.729 & 28 & 0.278 & 15 & 0.0402 & 4 & -129.19 \\
\hline 6 & 12 & 0.765 & 8 & 0.258 & 18 & 0.0322 & 15 & -130.48 \\
\hline 7 & 21 & 0.771 & 29 & 0.257 & 3 & 0.0295 & 23 & -132.33 \\
\hline 8 & 27 & 0.809 & 21 & 0.252 & 27 & 0.0250 & 21 & -134.15 \\
\hline 9 & 24 & 0.830 & 12 & 0.244 & 16 & 0.0238 & 24 & -143.67 \\
\hline 10 & 16 & 0.831 & 3 & 0.223 & 24 & 0.0219 & 16 & -145.65 \\
\hline 11 & 23 & 0.857 & 24 & 0.208 & 26 & 0.0154 & 18 & -173.16 \\
\hline 12 & 3 & 0.866 & 23 & 0.180 & 21 & 0.0150 & 3 & -184.19 \\
\hline 13 & 28 & 0.871 & 25 & 0.175 & 28 & 0.0065 & 27 & -212.69 \\
\hline 14 & 29 & 0.902 & 26 & 0.147 & 25 & 0.0057 & 29 & -286.8 \\
\hline 15 & 18 & 0.906 & 7 & 0.120 & 23 & 0.0053 & 28 & -287.12 \\
\hline 16 & 25 & 0.927 & 18 & 0.104 & 29 & 0.0031 & 26 & -292.57 \\
\hline 17 & 26 & 0.930 & 16 & 0.082 & 20 & 0.0014 & 25 & -304.72 \\
\hline
\end{tabular}

On the table above, a darker highlight represents the first set of similar rankings. A lighter shade, distinguishes another set of similar rankings. For this load case, the VCPI and VSI rank bus 20 as the most critical bus, while Modal and QV rank bus 12 the most critical bus following by buses $7 \& 8$. Bus 27 shares the $8^{\text {th }}$ ranking for VSI and Modal methods, where as Bus 21 shares this ranking as designated by VCPI and QV methods. Lastly VSI and QV methods share ranks 9,10,12, and 14, for buses 27, 24, 16, 3, and 29 respectively.

Even when the system is marginally stable, it is still unclear as to what is the most critical bus. Despite some similarities between various methods, there isn't any general consensus between all four methods. However, from these results it can be seen that for a 
large system, determining a critical bus requires more factors as to what makes the bus critical. Since both QV and Modal analyses are closely related to reactive power, it is most likely that bus 12 and 7 are the most sensitive to changes in reactive power. Even though reactive power is an important contribution to the voltage stability problem, it may not necessarily be the sole cause of collapse. On the other hand it is unclear why bus 20 is defined the critical bus according to the indices. The fact that the VSI and VCPI also consider factors including apparent power, real power, and admittances, suggest that other quantities and their combinations can lead to a collapse. Thus, additional studies should be done in order to establish an appropriate relationship between instability and various quantities. 


\section{CHAPTER 6 CONCLUSION AND RECOMMENDATIONS}

This thesis investigated several key aspects of voltage stability using simulated synchrophasor data. Two indices from literature, VSI and VCPI were investigated in order to show how the synchrophasor data can be used to determine how far the entire system is from its voltage collapse point, as well as the weak points in a system. Steady state analysis was conducted using PowerWorld Simulators, and the indices were generated in MatLab. The performances of the indices were tested in the 10 bus- 3generator system, and a larger network consisting of 39 buses and 10 generators. Results show that their performances are coherent to each other with respect to voltage stability of the system. All indices fall between 0 and 1 when the system is stable. The VCPI can exceed 1 when the system is unstable.

When comparing the two studied indices, the predictive ability of the VSI is able to provide a more intuitive index compared to the VCPI. For almost all buses the VCPI does not give any predictable value at the voltage stability boundary. So they cannot estimate the voltage stability margin but may be used to determine critical lines or critical buses in a given load level. When looking at the global index, a .3 index margin should be considered which could allow operators to initiate the remedial actions necessary to prevent voltage collapse.

The characteristics of the two indices can be summed up as follows: 
Voltage collapse can be predicted from the index, and regardless of load type the index will still approach the same value. The curve shows a nonlinear trend near the voltage collapse point. Each index changes automatically with different operating conditions and does not depend on any particular scenario. Low voltages, and reactive margin alone conditions do not necessarily indicate a critical bus. Additional parameters such as powers, and system topology should be considered when making such an analysis.

One of the mains objectives of this thesis was investigate if the state of the system could be determined by looking at only the index values. Therefore a majority of the plots presented only show the index values per a given variable. It may be useful to plot voltage values along side with the indices in order to define other relationships between indices and voltage.

Since this thesis investigated these two indices using a steady state analysis approach, a natural extension to this study would be to conduct similar studies using a time-based dynamic analysis approach. As mentioned in the early chapters, this would require an accurate model of the system that represents the transients associated with a voltage collapse. A dynamic simulation can demonstrate the time-response of the system voltage with respect to a series of events. Therefore, helping to identify whether the system voltage is stable or not. Using this approach, an analysis can reveal the actual mechanisms of voltage instability and how the indices respond to it. 


\section{WORKS CITED}

[1] DOE's Office of Electricity Delivery and Energy Reliability, "The Smart Grid: An Introduction”, URL: http://www.oe.energy.gov/1165.htm

[2] Altuve, Ferrer Hector J., and Edmund O. Schweitzer. Modern Solutions for

Protection, Control, and Monitoring of Electric Power Systems. Pullman, Wash. (2350

NE Hopkins Court, Pullman, WA 99163 USA): Schweitzer Engineering Laboratories, 2010. Print

[3]S. Massuco et al, "Evaluation of some indices for voltage stability assessment", IEEE Bucharest Power Tech Conference, 28th June-2nd July, Bucharest, Romania, pp.1-8.

[4]Reis, C.; Barbosa, F.P.M., "A comparison of voltage stability indices,"

Electrotechnical Conference, 2006. MELECON 2006. IEEE Mediterranean, vol., no., pp.1007,1010, 16-19 May 2006

[5]Cupelli, M.; Doig Cardet, C.; Monti, A., "Voltage stability indices comparison on the IEEE-39 bus system using RTDS," Power System Technology (POWERCON), 2012 IEEE International Conference on, vol., no., pp.1,6, Oct. 30 2012-Nov. 22012

[6]Massucco, S.; Grillo, S.; Pitto, A.; Silvestro, F., "Evaluation of some indices for voltage stability assessment," PowerTech, 2009 IEEE Bucharest, vol., no., pp.1,8, June 28 2009-July 22009

[7] Kundur, P., Neal J. Balu, and Mark G. Lauby. Power System Stability and Control. New York: McGraw-Hill, 1994. Print.

[8] Taylor, Carson W., Neal J. Balu, and Dominic Maratukulam. Power System Voltage Stability. New York: McGraw-Hill, 1994. Print.

[9] Ajjarapu, Venkataramana. Computational Techniques for Voltage Stability Assessment and Control. New York, NY, USA: Springer, 2006. Print.

[10] Pal, M.K.; , "Voltage stability conditions considering load characteristics," Power Systems, IEEE Transactions on , vol.7, no.1, pp.243-249, Feb 1992

[11] Glover, J. Duncan., and Mulukutla S. Sarma. Power System Analysis and Design. Boston: PWS Pub., 1994. Print. 
[12] C. Canizares, "Voltage Stability Assessment: Concepts, Practices and Tools," IEEE/PES, 2003]

[13] A.C. Zambroni de Souza, Fritz W. Mohn, Isabella F. Borges, Tito R. Ocariz, Using $\mathrm{PV}$ and QV curves with the meaning of static contingency screening and planning, Electric Power Systems Research, Volume 81, Issue 7, July 2011, Pages 1491-1498]

[14] "IEEE Standard for Synchrophasor Measurements for Power Systems," IEEE Std C37.118.1-2011 (Revision of IEEE Std C37.118-2005), vol., no., pp.1-61, Dec. 282011

[15]Moxley, Roy. Synchrophasors in the Real World. Tech. Pullman: Schweitzer Engineering Laboratories, 2006. Print.

[16] Gao, B.; Morison, G.K.; Kundur, P., "Voltage Stability Evaluation Using Modal Analysis," Power Engineering Review, IEEE , vol.12, no.11, pp.41,, November 1992 doi: 10.1109/MPER.1992.161430

[17]Sharma, C.; Ganness, M.G., "Determination of the applicability of using modal analysis for the prediction of voltage stability," Transmission and Distribution Conference and Exposition, 2008. T\&D. IEEE/PES , vol., no., pp.1,7, 21-24 April 2008

[18]El-Sheikhi, F.A.; Saad, Y.M.; Osman, S.O.; El-Arroudi, K.M., "Voltage stability assessment using modal analysis of power systems including flexible AC transmission system (FACTS),"Power Engineering, 2003 Large Engineering Systems Conference on, vol., no., pp.105,108, 7-9 May 2003

[19] Rios, M.A.; Zapata, C. J.; Gomez, O.; Sanchez, J.L., "Voltage Stability assessment with Ranking of Contingencies using QV sensibility," Latin America Transactions, IEEE (Revista IEEE America Latina), vol.7, no.6, pp.665,672, Dec. 2009

[20] Salehi, V.; Mohammed, O.; , "Real-time voltage stability monitoring and evaluation using synchorophasors," North American Power Symposium (NAPS), 2011 , vol., no., pp.1-7, 4-6 Aug. 2011

[21] Moghavvemi, M.; Omar, F.M.; , "Technique for contingency monitoring and voltage collapse prediction," Generation, Transmission and Distribution, IEE Proceedings- , vol.145, no.6, pp.634-640, Nov 1998

[22] A. Berizzi, P. Finazzi, D. Dosi, P. Marannino and S. Corsi, "First and second order methods for voltage collapse assessment and security enhancement", IEEE Trans. on Power Systems, vol. 13, no. L10 \& L11 2, May 1998, pp. 543-551

[23] K. Chen, A. Hussein, M. E. Bradley and H. Wan, "A performance-index guided continuation method for fast computation for saddle-node bifurcation in power systems", IEEE Trans. on Power Systems, vol. 18, no. 2, May 2003, pp. 753-760. 
[24] De La Ree, J.; Centeno, V.; Thorp, J.S.; Phadke, A.G.; , "Synchronized Phasor Measurement Applications in Power Systems," Smart Grid, IEEE Transactions on, vol.1, no.1, pp.20-27, June 2010

[25]M. Zima, M. Larsson, P. Korba, C. Rehtanz and G. Andersson, "Design aspects for wide-area monitoring and control systems", Proceedings of the IEEE, Vol. 93, No. 5, May 2005, pp.980-996.

[26] Li, W.; Wang, Y.; Chen, T., "Investigation on the Thevenin equivalent parameters for online estimation of maximum power transfer limits," Generation, Transmission \& Distribution, IET , vol.4, no.10, pp.1180,1187, October 2010

[27]Yanfeng Gong; Schulz, N.; Guzman, A., "Synchrophasor-Based Real-Time Voltage Stability Index," Power Systems Conference and Exposition, 2006. PSCE '06. 2006 IEEE PES , vol., no., pp.1029,1036, Oct. 29 2006-Nov. 12006

[28] M. Moghavvemi, "New method for indicating voltage stability in power system" Proceedings of IEEE International Conference on Power Engineering, Singapore, IPEC, 1997, pp. 223-227.

[29] I. Musirin and T.K.A. Rahman, "Novel Fast Voltage Stability Index (FVSI) for Voltage Stability Analysis in Power Transmission System", 2002 Student Conference on Research and Development Proceedings, Shah Alam, Malasia, July 2002.

[30] A. Mohamed, G. B. Jasmon and S. Yusoff, "A static voltage collapse indicator using line stability factors", Journal of Industrial Technology, Vol. 7, No. 1, 1989, pp. 73-85.

[31] V. Balamourougan, T. S. Sidhu, and M. S. Sachdev, "Technique for online prediction of voltage collapse," IEEE Proceedings in Generation, Transmission and Distribution, Vol. 151, No. 4, July 2004, pp. 453-460.

[32]Hart, E.K.; Stoutenburg, E.D.; Jacobson, M.Z.; , "The Potential of Intermittent Renewables to Meet Electric Power Demand: Current Methods and Emerging Analytical Techniques," Proceedings of the IEEE, vol.100, no.2, pp.322-334,

[33]Weng, Z. X.; Shi, L. B.; Xu, Z.; Yao, L. Z.; Ni, Y. X.; Bazargan, M.; , "Effects of wind power variability and intermittency on power flow," Power and Energy Society General Meeting, 2012 IEEE, vol., no., pp.1-7, 22-26 July 2012]

[34] [J.G. Slootweg, W.L. Kling, "Aggregated modeling of wind parks in power system dynamics simulations," Power Tech Conference Proceedings, 2003 IEEE Bologna.]

[35] Konopinski, Ryan, "Voltage security assessment with high penetration levels of utility-scale doubly fed induction generator wind plants" (2009). Graduate Theses and Dissertations. Paper 10606. 


\section{APPENDIX A- Matlab Code}

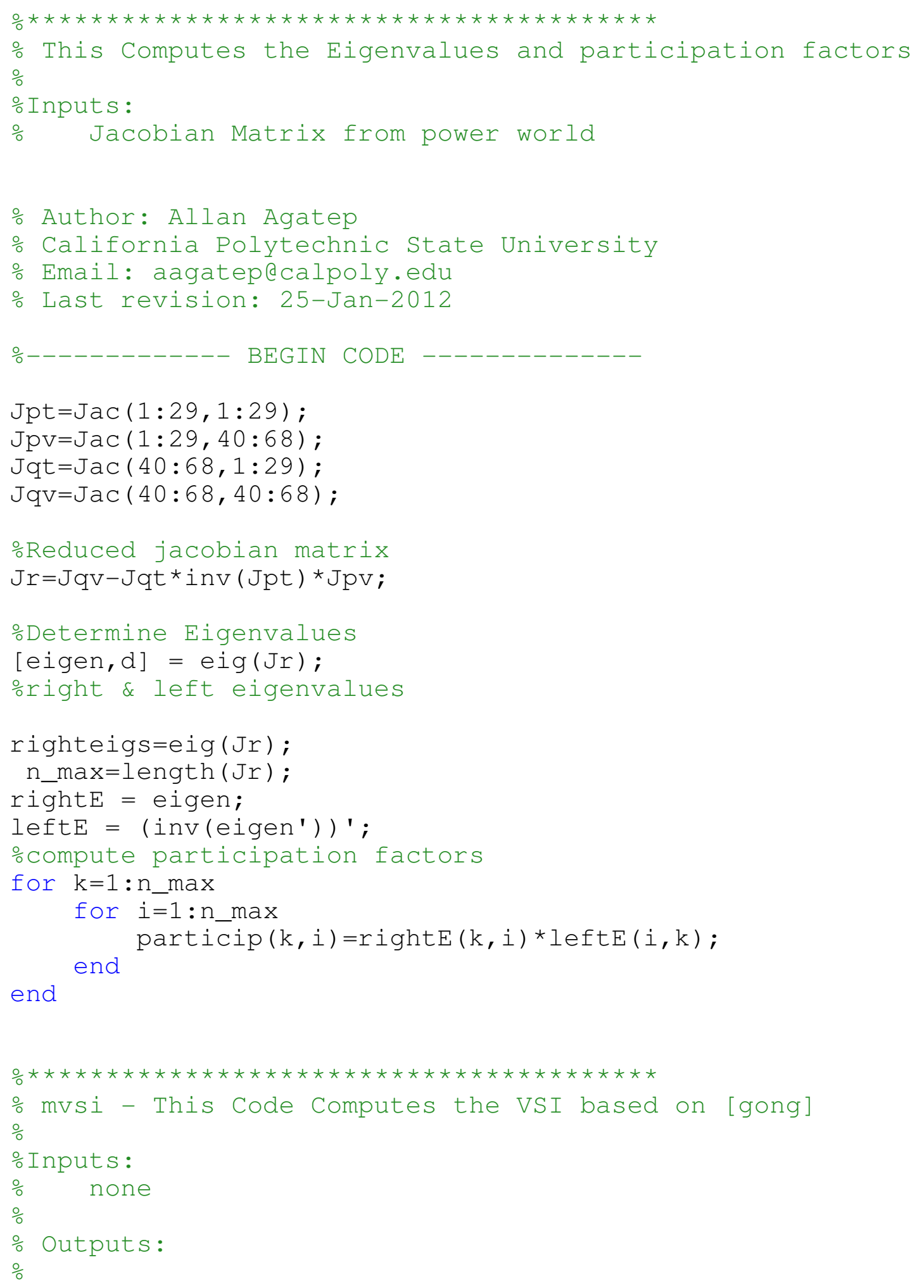




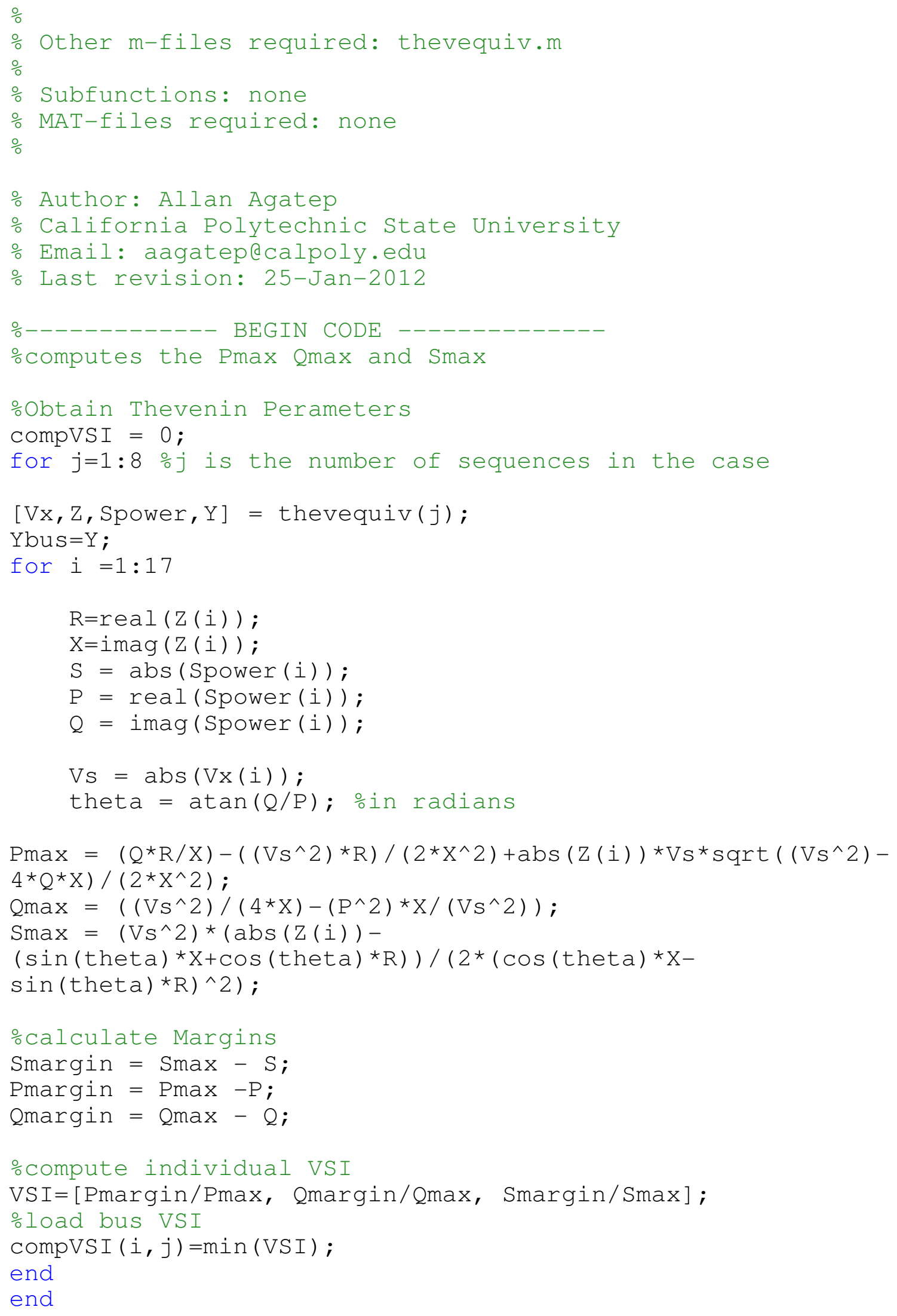




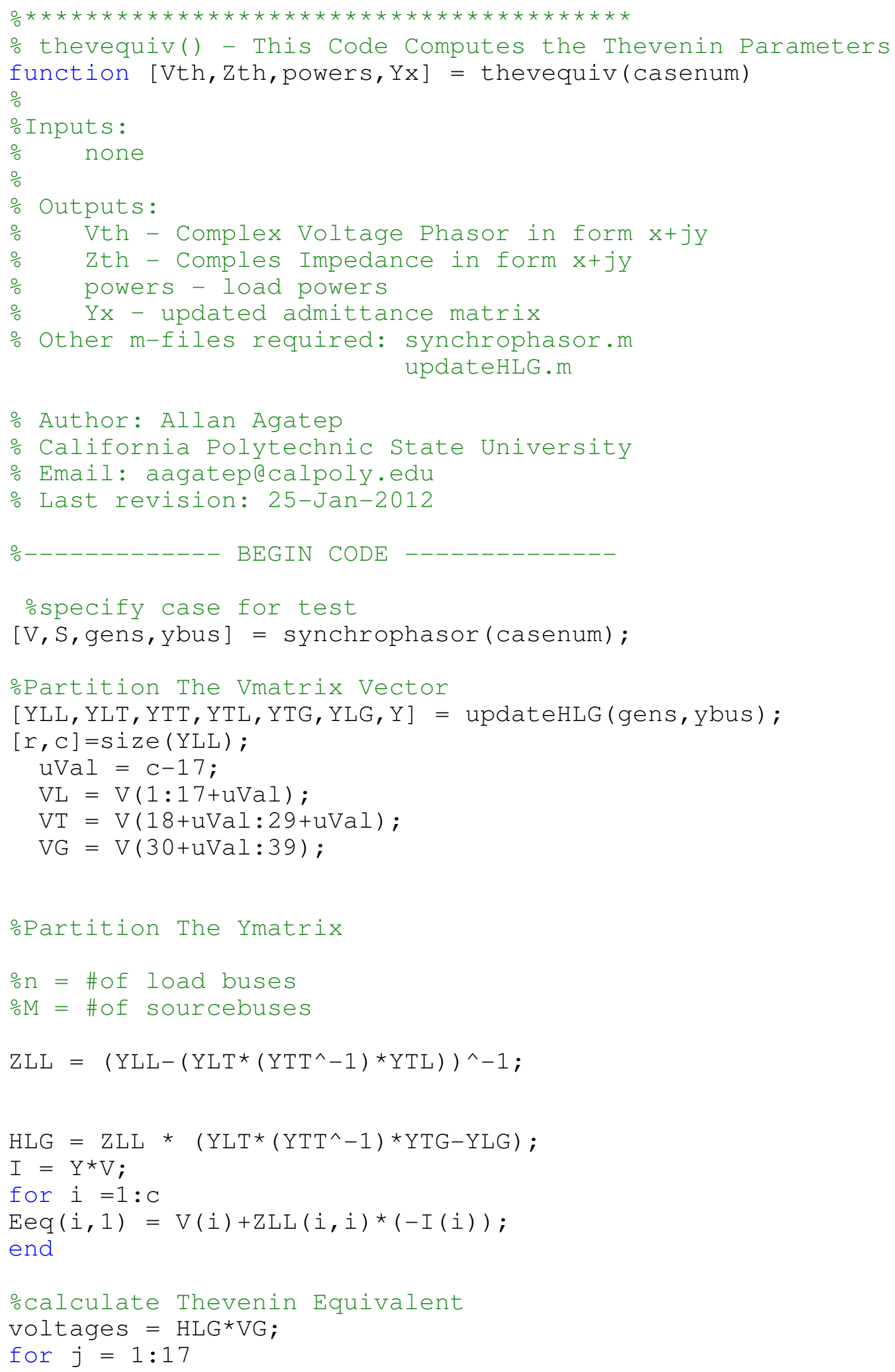




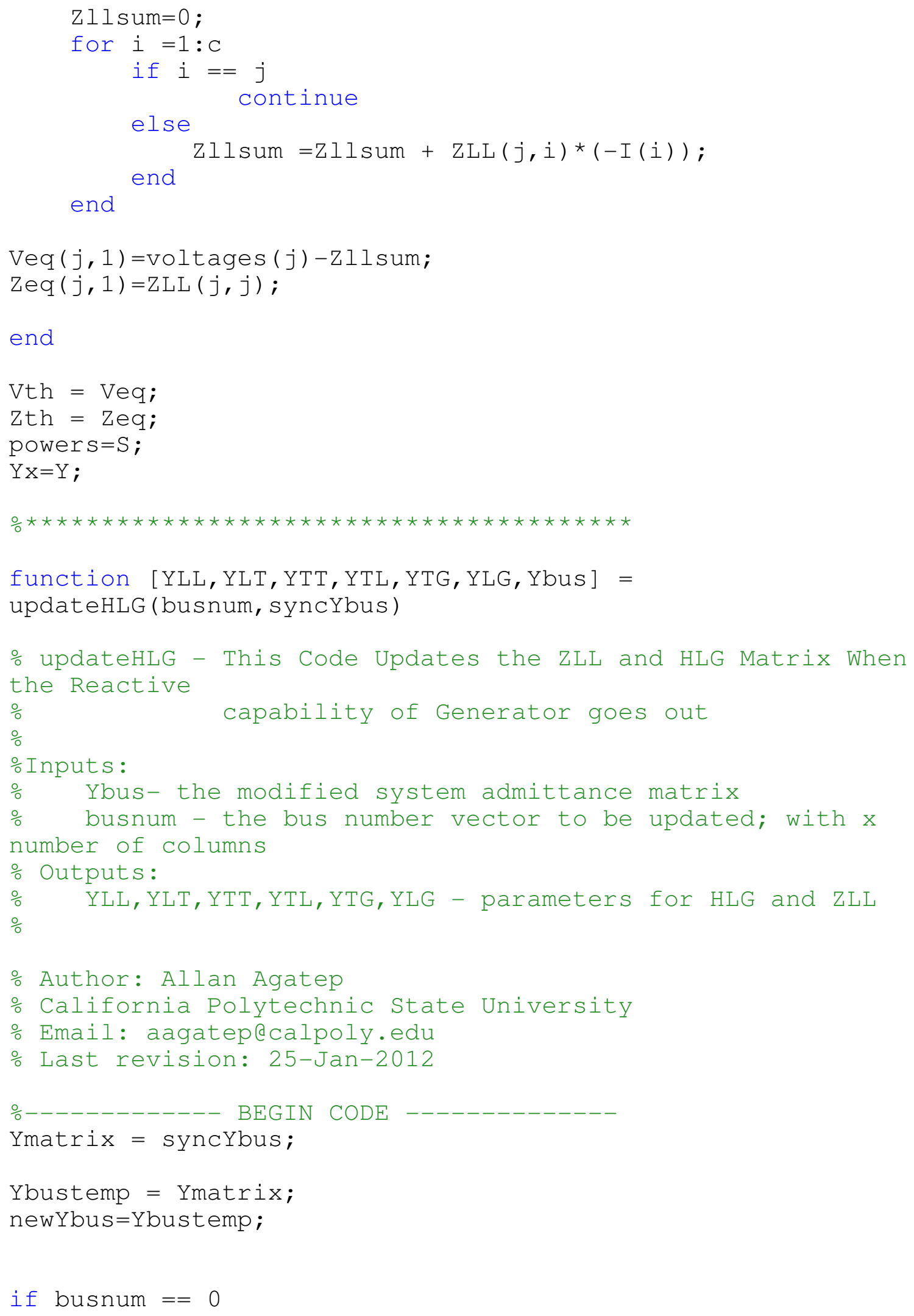




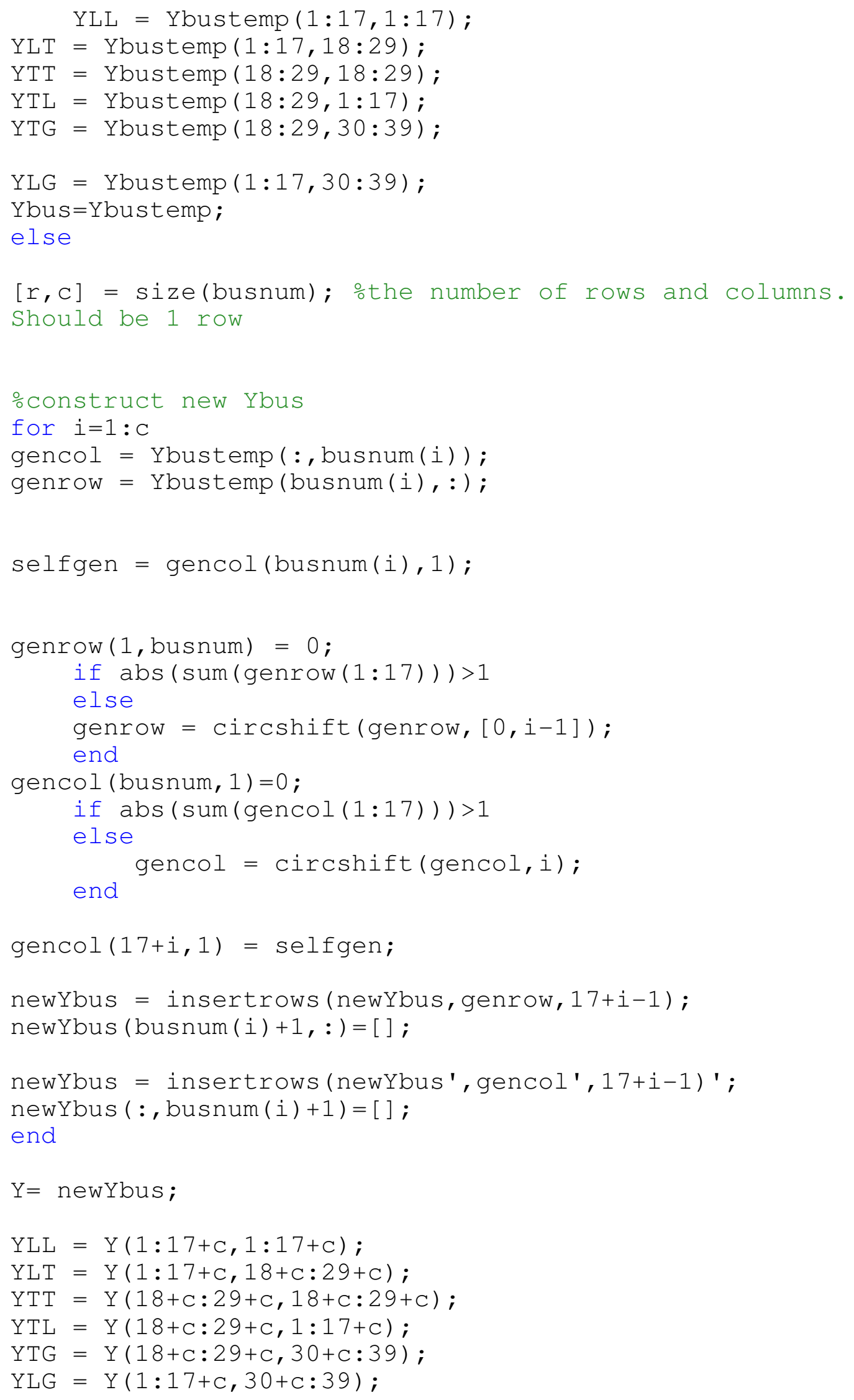




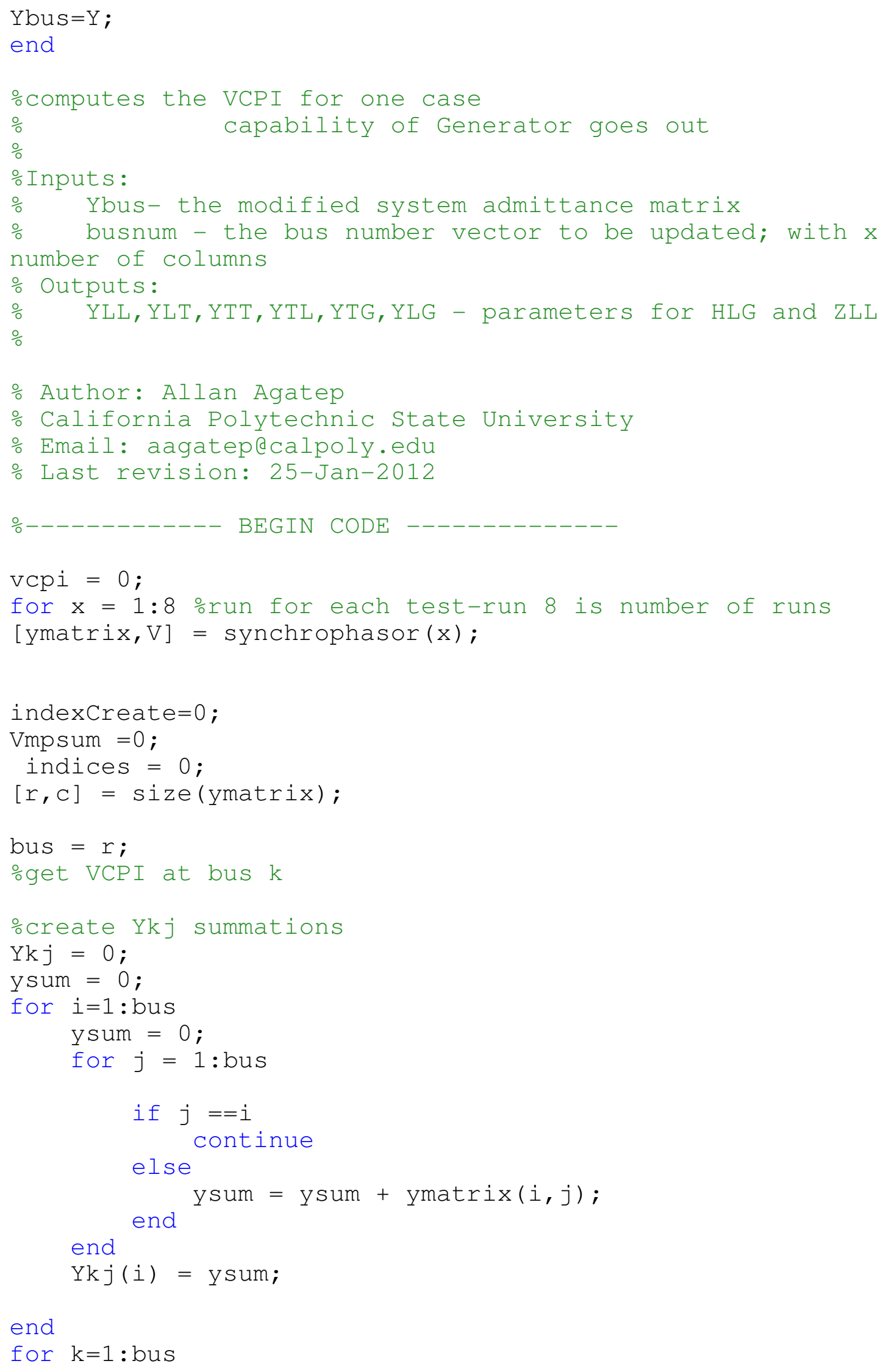




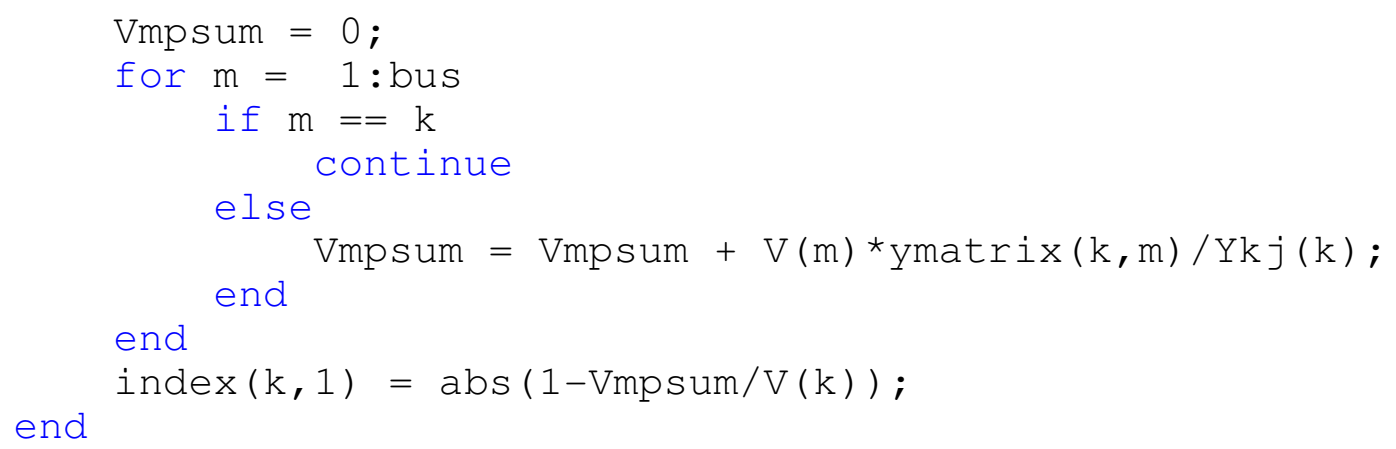




\section{APPENDIX B- Power Flow and Line Loses for Case 3}

\begin{tabular}{|c|c|c|c|c|c|c|c|c|c|}
\hline \multicolumn{2}{|c|}{ Branch } & \multicolumn{4}{|c|}{ Before } & \multicolumn{4}{|c|}{ After } \\
\hline FROM & TO & $\begin{array}{l}\text { Line } \\
\text { MW }\end{array}$ & $\begin{array}{l}\text { Line } \\
\text { MVR }\end{array}$ & $\begin{array}{l}\text { Line } \\
\text { MVA }\end{array}$ & $\begin{array}{l}\text { Loss } \\
\text { MVR }\end{array}$ & $\begin{array}{l}\text { Line } \\
\text { MW }\end{array}$ & $\begin{array}{l}\text { Line } \\
\text { MVR }\end{array}$ & $\begin{array}{l}\text { Line } \\
\text { MVA }\end{array}$ & $\begin{array}{l}\text { Loss } \\
\text { MVR }\end{array}$ \\
\hline 1 & 2 & -51.2 & -26.6 & 57.7 & -75.3 & -14.7 & -24.8 & 28.8 & -75.93 \\
\hline 1 & 39 & 51.2 & 26.6 & 57.7 & -79.22 & 14.7 & 24.8 & 28.8 & -79.76 \\
\hline 10 & 13 & 490.6 & 49 & 493 & 3.49 & 0 & 0 & 0 & 0 \\
\hline 10 & 32 & -650 & -193 & 678.1 & 106.95 & -650 & -191.7 & 677.7 & 108.33 \\
\hline 10 & 11 & 159.4 & 144.1 & 214.9 & -5.06 & 650 & 191.7 & 677.7 & 13.4 \\
\hline 12 & 11 & -20.4 & -46.7 & 51 & 1.22 & -178.6 & -54.6 & 186.7 & 17.06 \\
\hline 12 & 13 & 11.8 & -54.5 & 55.8 & 1.46 & 169.9 & -46.6 & 176.2 & 15.19 \\
\hline 13 & 14 & 501.4 & -10.5 & 501.5 & 9.23 & 169.4 & -61.8 & 180.3 & -12.68 \\
\hline 14 & 15 & 262.4 & -79.7 & 274.3 & -19.64 & 204 & -109.9 & 231.8 & -23.4 \\
\hline 15 & 16 & -106.9 & -236.1 & 259.1 & -11.35 & -164.9 & -262.5 & 310 & -8.24 \\
\hline 16 & 19 & -409.2 & -85.6 & 418.1 & 0.19 & -409.1 & -102.3 & 421.7 & 1.25 \\
\hline 16 & 17 & 171.6 & -36.1 & 175.4 & -11.33 & 113.1 & -29.8 & 117 & -12.63 \\
\hline 16 & 24 & 24.5 & -129.7 & 132 & -6.16 & 24.5 & -137.2 & 139.4 & -5.97 \\
\hline 16 & 21 & -272.7 & -10.5 & 272.9 & -16.79 & -272.7 & -22 & 273.6 & -16.45 \\
\hline 17 & 27 & 77.7 & -50.5 & 92.7 & -32.4 & 61.4 & -54.9 & 82.4 & -32.38 \\
\hline 17 & 18 & 93.7 & 25.8 & 97.2 & -12.89 & 51.6 & 37.7 & 63.9 & -13.14 \\
\hline 19 & 20 & 217.1 & 22.2 & 218.2 & 6.82 & 217.1 & 16.6 & 217.7 & 6.81 \\
\hline 19 & 33 & -629 & -107.9 & 638.2 & 61.12 & -629 & -120.2 & 640.3 & 61.76 \\
\hline 2 & 30 & -250 & -158.2 & 295.8 & 15.27 & -250 & -173.2 & 304.1 & 16.23 \\
\hline 2 & 3 & 329.9 & 161.5 & 367.3 & -7.93 & 350.2 & 190.8 & 398.8 & -4.08 \\
\hline 2 & 25 & -131.1 & 45.4 & 138.8 & -14.4 & -114.9 & 33.6 & 119.7 & -14.71 \\
\hline 20 & 34 & -505.4 & -103.1 & 515.9 & 51.01 & -505.4 & -108.6 & 517 & 51.35 \\
\hline 21 & 22 & -588.4 & -126 & 601.7 & 20.91 & -588.3 & -137.8 & 604.3 & 21.79 \\
\hline 22 & 35 & -650 & -192.7 & 678 & 63.39 & -650 & -206.2 & 681.9 & 64.38 \\
\hline 22 & 23 & 58.8 & 45.9 & 74.6 & -19.42 & 58.9 & 46.6 & 75.1 & -19.34 \\
\hline 23 & 36 & -558.5 & -47.7 & 560.6 & 79.29 & -558.5 & -55.3 & 561.3 & 79.81 \\
\hline 23 & 24 & 332.7 & 15.7 & 333.1 & -2.09 & 332.7 & 24 & 333.6 & -1.48 \\
\hline 25 & 37 & -538.3 & 24.2 & 538.9 & 64.4 & -538.3 & 11.6 & 538.5 & 64.66 \\
\hline 25 & 26 & 148.3 & -18.7 & 149.5 & -51.5 & 164.9 & -17.7 & 165.8 & -49.56 \\
\hline 26 & 29 & -157.7 & -40.5 & 162.8 & -97.59 & -157.7 & -43.9 & 163.7 & -97.05 \\
\hline 26 & 28 & -100.9 & -35.3 & 106.9 & -80.35 & -100.9 & -38.7 & 108.1 & -79.9 \\
\hline 26 & 27 & 246.4 & 89 & 262 & -15.94 & 262.8 & 95 & 279.5 & -14.36 \\
\hline 28 & 29 & -338.2 & 13.3 & 338.5 & -11.21 & -338.2 & 9.4 & 338.4 & -11.1 \\
\hline 29 & 38 & -824.7 & 50.7 & 826.3 & 102.39 & -824.7 & 42.8 & 825.9 & 102.52 \\
\hline 3 & 18 & 88.2 & -25.2 & 91.7 & -21.04 & 130.3 & -35.8 & 135.1 & -19.47 \\
\hline 3 & 4 & -130.2 & 191.9 & 232 & -9.89 & -152.4 & 227.9 & 274.2 & -4.61 \\
\hline 4 & 14 & -236.1 & -65.2 & 245 & -5.24 & 35 & -72.9 & 80.9 & -12.07 \\
\hline 4 & 5 & -469.9 & 55.5 & 473.1 & 17.52 & -763.4 & 93.8 & 769.2 & 69.93 \\
\hline 5 & 8 & 374.5 & 61.3 & 379.4 & 3.33 & 365.2 & 52 & 368.9 & 3.23 \\
\hline 5 & 6 & -846.2 & -23.3 & 846.5 & 15.58 & -1133.7 & -28.1 & 1134.1 & 32.37 \\
\hline 6 & 31 & -1261 & -27.9 & 1261.3 & 478.82 & -1267.8 & -61.9 & 1269.3 & 497.9 \\
\hline 6 & 11 & -138.5 & -112 & 178.1 & -10.73 & -467.2 & -99.5 & 477.7 & 7.07 \\
\hline 6 & 7 & 551.8 & 101 & 560.9 & 19.93 & 598.4 & 100.9 & 606.9 & 26.36 \\
\hline 7 & 8 & 280.9 & -15.5 & 281.3 & -3.31 & 327.2 & -22.1 & 327.9 & -1.57 \\
\hline 8 & 9 & 53.5 & -156.6 & 165.5 & -28.49 & 90.4 & -174.1 & 196.2 & -23.3 \\
\hline 9 & 39 & 53 & -128.2 & 138.7 & -123.6 & 89.5 & -150.8 & 175.4 & -120.71 \\
\hline
\end{tabular}




\section{APPENDIX C- QV Analysis Calculations for IEEE-39 Bus System}

\begin{tabular}{|r|c|c|c|c|c|c|}
\hline \multirow{3}{*}{$\begin{array}{l}\text { Bus } \\
\text { Number }\end{array}$} & \multicolumn{6}{|c|}{ Reactive Margin [MVAR] } \\
\cline { 2 - 7 } & $\begin{array}{c}\text { Load } \\
\text { Factor }=\end{array}$ & $\begin{array}{c}\text { Load } \\
\text { Factor }= \\
1.05\end{array}$ & $\begin{array}{c}\text { Load } \\
\text { Factor }= \\
1.10\end{array}$ & $\begin{array}{c}\text { Load } \\
\text { Factor }= \\
1.15\end{array}$ & $\begin{array}{c}\text { Load } \\
\text { Factor }= \\
1.20\end{array}$ & $\begin{array}{c}\text { Load } \\
\text { Factor }= \\
1.25\end{array}$ \\
\hline 3 & -1087.59 & -1017.63 & -912.03 & -758.12 & -537.5 & -184.19 \\
\hline 4 & -1043.78 & -957.24 & -829.07 & -654.45 & -425.6 & -129.19 \\
\hline 7 & -1008.73 & -920.28 & -793.68 & -623.51 & -401.46 & -118.71 \\
\hline 8 & -1002.09 & -915.2 & -790.37 & -621.92 & -401.4 & -119.39 \\
\hline 12 & -595.89 & -567.52 & -522.09 & -446.45 & -321.55 & -106.88 \\
\hline 15 & -811.21 & -760.08 & -681.33 & -565.13 & -394.18 & -130.48 \\
\hline 16 & -811.01 & -768.58 & -696.87 & -585.29 & -417.33 & -145.65 \\
\hline 18 & -986.68 & -927.21 & -835.05 & -697.58 & -497.28 & -173.16 \\
\hline 20 & -460.83 & -439.85 & -410.58 & -369.98 & -298.95 & -127.08 \\
\hline 21 & -633.1 & -603.2 & -558.83 & -485.94 & -363.45 & -134.15 \\
\hline 23 & -559.8 & -534.34 & -497.55 & -442.66 & -341.8 & -132.33 \\
\hline 24 & -745.75 & -710.44 & -651.75 & -555.81 & -403.18 & -143.67 \\
\hline 25 & -1074.78 & -1025.02 & -943.96 & -812.67 & -617.68 & -304.72 \\
\hline 26 & -890.19 & -860.33 & -812.23 & -739.28 & -611.46 & -292.57 \\
\hline 27 & -917.6 & -874.61 & -813.5 & -725.03 & -563.63 & -212.69 \\
\hline 28 & -512.83 & -507.05 & -497.29 & -479.25 & -443.93 & -287.12 \\
\hline 29 & -490.19 & -485.02 & -475.88 & -460.09 & -430.85 & -286.8 \\
\hline
\end{tabular}


APPENDIX D- Modal Analysis Calculations for IEEE-39 Bus System

\begin{tabular}{|c|c|c|}
\hline \multirow[t]{2}{*}{ Mode } & \multicolumn{2}{|c|}{ Eigenvalue } \\
\hline & Load Factor $=1.0$ & Load Factor $=1.25$ \\
\hline$\lambda_{1}$ & 1034.70 & 922.97 \\
\hline$\lambda_{z}$ & 785.56 & 709.81 \\
\hline$\lambda_{\mathrm{a}}$ & 674.14 & 646.51 \\
\hline$\lambda_{4}$ & 525.53 & 468.90 \\
\hline$\lambda_{\mathrm{s}}$ & 410.53 & 377.28 \\
\hline$\lambda_{s}$ & 393.17 & 369.79 \\
\hline$\lambda_{z}$ & 354.38 & 344.50 \\
\hline$\lambda_{\mathrm{g}}$ & 350.79 & 342.66 \\
\hline$\lambda_{g}$ & 332.44 & 300.99 \\
\hline$\lambda_{10}$ & 232.49 & 224.36 \\
\hline$\lambda_{11}$ & 229.17 & 217.43 \\
\hline$\lambda_{1 z}$ & 220.38 & 203.93 \\
\hline$\lambda_{1 s}$ & 208.95 & 200.25 \\
\hline$\lambda_{14}$ & 194.97 & 192.36 \\
\hline$\lambda_{15}$ & 176.08 & 166.01 \\
\hline$\lambda_{16}$ & 152.08 & 147.16 \\
\hline$\lambda_{17}$ & 137.24 & 8.39 \\
\hline$\lambda_{18}$ & 9.73 & 125.85 \\
\hline$\lambda_{19}$ & 19.83 & 18.44 \\
\hline$\lambda_{20}$ & 112.92 & 107.54 \\
\hline$\lambda_{21}$ & 32.90 & 31.71 \\
\hline$\lambda_{2 \pi}$ & 39.21 & 34.81 \\
\hline$\lambda_{23}$ & 50.40 & 49.00 \\
\hline$\lambda_{24}$ & 96.90 & 93.07 \\
\hline$\lambda_{2 \mathrm{~s}}$ & 62.61 & 61.67 \\
\hline$\lambda_{26}$ & 64.88 & 60.53 \\
\hline$\lambda_{27}$ & 86.27 & 81.34 \\
\hline
\end{tabular}




\begin{tabular}{|l|l|l|}
\hline$\lambda_{2 \mathrm{~g}}$ & 80.02 & 75.82 \\
\hline$\lambda_{29}$ & 76.34 & 73.65 \\
\hline
\end{tabular}

\begin{tabular}{|c|c|c|c|c|c|c|c|}
\hline \multirow[b]{2}{*}{ Bus } & \multicolumn{7}{|c|}{ Bus Participation Factors, (Load Factor $=1)$} \\
\hline & $\lambda_{1}$ & $\lambda_{2}$ & $\lambda_{\mathrm{s}}$ & $\lambda_{a}$ & $\lambda_{\mathbf{5}}$ & $\lambda_{\mathrm{f}}$ & $\lambda_{\pi}$ \\
\hline 1 & 0.00000 & 0.00000 & 0.00000 & 0.00000 & 0.00000 & 0.00050 & 0.00354 \\
\hline 2 & 0.00000 & 0.00000 & 0.00001 & 0.00000 & 0.00048 & 0.08034 & 0.44594 \\
\hline 3 & 0.00001 & 0.00001 & 0.00018 & 0.00004 & 0.00112 & 0.11361 & 0.06380 \\
\hline 4 & 0.00346 & 0.00099 & 0.00000 & 0.00145 & 0.00114 & 0.01811 & 0.03054 \\
\hline 5 & 0.38602 & 0.02208 & 0.00000 & 0.01447 & 0.08448 & 0.00577 & 0.00633 \\
\hline 6 & 0.53326 & 0.00508 & 0.00000 & 0.02107 & 0.01046 & 0.00418 & 0.00732 \\
\hline 7 & 0.02248 & 0.00155 & 0.00000 & 0.45672 & 0.01401 & 0.00060 & 0.00084 \\
\hline 8 & 0.01564 & 0.00230 & 0.00000 & 0.49269 & 0.00002 & 0.00061 & 0.00152 \\
\hline 9 & 0.00001 & 0.00000 & 0.00000 & 0.00183 & 0.00000 & 0.00000 & 0.00001 \\
\hline 10 & 0.00860 & 0.58204 & 0.00005 & 0.00119 & 0.00041 & 0.00090 & 0.00219 \\
\hline 11 & 0.02943 & 0.18111 & 0.00003 & 0.00461 & 0.36721 & 0.00639 & 0.00025 \\
\hline 12 & 0.00002 & 0.00073 & 0.00000 & 0.00000 & 0.00000 & 0.00000 & 0.00001 \\
\hline 13 & 0.00105 & 0.19693 & 0.00000 & 0.00477 & 0.37750 & 0.00412 & 0.00026 \\
\hline 14 & 0.00000 & 0.00712 & 0.00037 & 0.00112 & 0.13107 & 0.00238 & 0.01183 \\
\hline 15 & 0.00000 & 0.00005 & 0.03276 & 0.00001 & 0.00440 & 0.00793 & 0.00058 \\
\hline 16 & 0.00000 & 0.00002 & 0.73872 & 0.00000 & 0.00000 & 0.03093 & 0.00837 \\
\hline 17 & 0.00000 & 0.00000 & 0.09013 & 0.00000 & 0.00455 & 0.36268 & 0.13648 \\
\hline 18 & 0.00000 & 0.00000 & 0.00677 & 0.00001 & 0.00283 & 0.28546 & 0.03140 \\
\hline 19 & 0.00000 & 0.00000 & 0.00903 & 0.00000 & 0.00000 & 0.00240 & 0.00107 \\
\hline 20 & 0.00000 & 0.00000 & 0.00015 & 0.00000 & 0.00000 & 0.00016 & 0.00010 \\
\hline 21 & 0.00000 & 0.00000 & 0.01621 & 0.00000 & 0.00000 & 0.00391 & 0.00446 \\
\hline 22 & 0.00000 & 0.00000 & 0.00035 & 0.00000 & 0.00000 & 0.00067 & 0.00841 \\
\hline 23 & 0.00000 & 0.00000 & 0.00022 & 0.00000 & 0.00000 & 0.00009 & 0.00156 \\
\hline 24 & 0.00000 & 0.00000 & 0.10387 & 0.00000 & 0.00000 & 0.02747 & 0.00997 \\
\hline 25 & 0.00000 & 0.00000 & 0.00000 & 0.00000 & 0.00010 & 0.01996 & 0.19247 \\
\hline 26 & 0.00000 & 0.00000 & 0.00002 & 0.00000 & 0.00001 & 0.00049 & 0.01281 \\
\hline 27 & 0.00000 & 0.00000 & 0.00112 & 0.00000 & 0.00023 & 0.02034 & 0.01786 \\
\hline 28 & 0.00000 & 0.00000 & 0.00000 & 0.00000 & 0.00000 & 0.00000 & 0.00006 \\
\hline 29 & 0.00000 & 0.00000 & 0.00000 & 0.00000 & 0.00000 & 0.00000 & 0.00003 \\
\hline
\end{tabular}




\begin{tabular}{|r|c|c|c|c|c|c|c|}
\hline \multirow{2}{*}{ Bus } & \multicolumn{7}{|c|}{ Bus Participation Factors, (Load Factor $=1)$} \\
\cline { 2 - 8 } & $\lambda_{\mathbf{g}}$ & $\lambda_{\mathbf{9}}$ & $\lambda_{1 \mathbf{0}}$ & $\lambda_{1 \mathbf{z}}$ & $\lambda_{1 \mathbf{z}}$ & $\lambda_{1 \mathbf{z}}$ & $\lambda_{1}$ \\
\hline 1 & 0.00004 & 0.00065 & 0.00043 & 0.00002 & 0.00042 & 0.00081 & 0.00001 \\
\hline 2 & 0.00441 & 0.06970 & 0.01749 & 0.00091 & 0.01490 & 0.02569 & 0.00021 \\
\hline 3 & 0.00039 & 0.00042 & 0.21380 & 0.01099 & 0.16306 & 0.05028 & 0.00014 \\
\hline 4 & 0.00014 & 0.26292 & 0.06416 & 0.00416 & 0.00035 & 0.00493 & 0.00005 \\
\hline 5 & 0.00002 & 0.13985 & 0.11468 & 0.00495 & 0.03820 & 0.00003 & 0.00000 \\
\hline 6 & 0.00003 & 0.12878 & 0.08387 & 0.00342 & 0.04395 & 0.00054 & 0.00000 \\
\hline 7 & 0.00000 & 0.02407 & 0.06826 & 0.00306 & 0.03367 & 0.00017 & 0.00000 \\
\hline 8 & 0.00001 & 0.03045 & 0.06557 & 0.00287 & 0.03732 & 0.00034 & 0.00000 \\
\hline 9 & 0.00000 & 0.00034 & 0.00190 & 0.00009 & 0.00126 & 0.00001 & 0.00000 \\
\hline 10 & 0.00001 & 0.04405 & 0.00263 & 0.00014 & 0.00774 & 0.00217 & 0.00001 \\
\hline 11 & 0.00000 & 0.01011 & 0.02529 & 0.00087 & 0.08194 & 0.00622 & 0.00001 \\
\hline 12 & 0.00000 & 0.00017 & 0.00006 & 0.00000 & 0.00021 & 0.00007 & 0.00000 \\
\hline 13 & 0.00001 & 0.00401 & 0.04858 & 0.00191 & 0.03097 & 0.00033 & 0.00000 \\
\hline 14 & 0.00004 & 0.22485 & 0.02551 & 0.00089 & 0.20058 & 0.01826 & 0.00004 \\
\hline 15 & 0.00007 & 0.01035 & 0.00572 & 0.00013 & 0.09339 & 0.01237 & 0.00006 \\
\hline 16 & 0.00033 & 0.00125 & 0.00017 & 0.00044 & 0.00000 & 0.00000 & 0.00000 \\
\hline 17 & 0.00186 & 0.00336 & 0.05678 & 0.00327 & 0.04480 & 0.02107 & 0.00004 \\
\hline 18 & 0.00069 & 0.00193 & 0.03799 & 0.00125 & 0.06816 & 0.01972 & 0.00031 \\
\hline 19 & 0.00004 & 0.00022 & 0.04076 & 0.63180 & 0.00008 & 0.00000 & 0.00000 \\
\hline 20 & 0.00000 & 0.00002 & 0.01650 & 0.27181 & 0.00004 & 0.00000 & 0.00000 \\
\hline 21 & 0.08031 & 0.00020 & 0.00075 & 0.00283 & 0.00011 & 0.00003 & 0.00000 \\
\hline 22 & 0.62783 & 0.00000 & 0.00033 & 0.00126 & 0.00006 & 0.00003 & 0.00000 \\
\hline 23 & 0.26529 & 0.00011 & 0.00051 & 0.00186 & 0.00009 & 0.00003 & 0.00000 \\
\hline 24 & 0.01609 & 0.00263 & 0.01194 & 0.04534 & 0.00196 & 0.00063 & 0.00000 \\
\hline 25 & 0.00201 & 0.03849 & 0.06930 & 0.00341 & 0.04592 & 0.04900 & 0.00025 \\
\hline 26 & 0.00016 & 0.00100 & 0.00108 & 0.00024 & 0.02329 & 0.49665 & 0.00254 \\
\hline 27 & 0.00024 & 0.00004 & 0.02594 & 0.00208 & 0.06683 & 0.27067 & 0.00233 \\
\hline 28 & 0.00000 & 0.00001 & 0.00001 & 0.00000 & 0.00019 & 0.00314 & 0.31433 \\
\hline 29 & 0.00000 & 0.00000 & 0.00001 & 0.00001 & 0.00049 & 0.01681 & 0.67966 \\
\hline & & & & & & & \\
\hline
\end{tabular}




\begin{tabular}{|r|c|c|c|c|c|c|c|}
\hline \multirow{2}{*}{ Bus } & \multicolumn{7}{|c|}{ Bus Participation Factors, (Load Factor $=1)$} \\
\cline { 2 - 8 } & $\lambda_{1 \mathbf{s}}$ & $\lambda_{1 \mathbf{6}}$ & $\lambda_{17}$ & $\lambda_{1 \mathbf{s}}$ & $\lambda_{1 \mathbf{g}}$ & $\lambda_{2 \mathbf{0}}$ & $\lambda_{2 \mathbf{1}}$ \\
\hline 1 & 0.00003 & 0.00000 & 0.00213 & 0.00166 & 0.00314 & 0.02105 & 0.00039 \\
\hline 2 & 0.00054 & 0.00003 & 0.01674 & 0.00840 & 0.01075 & 0.07071 & 0.00071 \\
\hline 3 & 0.00327 & 0.00009 & 0.01308 & 0.03311 & 0.01609 & 0.01107 & 0.00219 \\
\hline 4 & 0.13665 & 0.00114 & 0.29992 & 0.06308 & 0.00393 & 0.00104 & 0.00021 \\
\hline 5 & 0.01164 & 0.00003 & 0.00390 & 0.06183 & 0.02592 & 0.00030 & 0.00307 \\
\hline 6 & 0.00346 & 0.00000 & 0.00183 & 0.05778 & 0.02725 & 0.00000 & 0.00338 \\
\hline 7 & 0.01847 & 0.00006 & 0.00088 & 0.06689 & 0.03879 & 0.01401 & 0.00714 \\
\hline 8 & 0.01498 & 0.00004 & 0.00002 & 0.06465 & 0.03743 & 0.01335 & 0.00710 \\
\hline 9 & 0.00101 & 0.00000 & 0.00000 & 0.01450 & 0.01222 & 0.00529 & 0.00432 \\
\hline 10 & 0.00053 & 0.00008 & 0.12531 & 0.05385 & 0.02171 & 0.03772 & 0.00066 \\
\hline 11 & 0.00009 & 0.00007 & 0.12212 & 0.06100 & 0.02907 & 0.02591 & 0.00200 \\
\hline 12 & 0.00003 & 0.00001 & 0.01993 & 0.10847 & 0.08357 & 0.01249 & 0.01088 \\
\hline 13 & 0.00182 & 0.00003 & 0.04827 & 0.06319 & 0.01962 & 0.02936 & 0.00006 \\
\hline 14 & 0.00063 & 0.00009 & 0.09909 & 0.06462 & 0.00374 & 0.00011 & 0.00336 \\
\hline 15 & 0.30610 & 0.00094 & 0.02094 & 0.04410 & 0.01876 & 0.19962 & 0.05365 \\
\hline 16 & 0.01676 & 0.00002 & 0.01305 & 0.02789 & 0.03756 & 0.02533 & 0.05312 \\
\hline 17 & 0.00579 & 0.00011 & 0.02042 & 0.03481 & 0.06638 & 0.02789 & 0.01484 \\
\hline 18 & 0.03783 & 0.00011 & 0.01949 & 0.03792 & 0.05335 & 0.10565 & 0.01246 \\
\hline 19 & 0.00474 & 0.00000 & 0.00024 & 0.00455 & 0.00766 & 0.00046 & 0.01544 \\
\hline 20 & 0.00820 & 0.00001 & 0.00591 & 0.00170 & 0.00344 & 0.02062 & 0.00907 \\
\hline 21 & 0.00562 & 0.58948 & 0.00111 & 0.01773 & 0.03247 & 0.00161 & 0.07698 \\
\hline 22 & 0.02631 & 0.00112 & 0.01278 & 0.00581 & 0.01257 & 0.03356 & 0.03781 \\
\hline 23 & 0.02754 & 0.30549 & 0.02045 & 0.00629 & 0.01371 & 0.04341 & 0.04178 \\
\hline 24 & 0.36047 & 0.10081 & 0.05326 & 0.02606 & 0.04058 & 0.05478 & 0.07219 \\
\hline 25 & 0.00307 & 0.00014 & 0.05158 & 0.00691 & 0.01786 & 0.20886 & 0.00666 \\
\hline 26 & 0.00419 & 0.00005 & 0.01140 & 0.01951 & 0.10537 & 0.01113 & 0.08130 \\
\hline 27 & 0.00000 & 0.00003 & 0.01515 & 0.03107 & 0.12330 & 0.02280 & 0.01796 \\
\hline 28 & 0.00021 & 0.00000 & 0.00075 & 0.00861 & 0.09161 & 0.00139 & 0.31783 \\
\hline 29 & 0.00001 & 0.00000 & 0.00024 & 0.00402 & 0.04214 & 0.00048 & 0.14345 \\
\hline
\end{tabular}




\begin{tabular}{|c|c|c|c|c|c|c|c|c|}
\hline \multirow[b]{2}{*}{ Bus } & \multicolumn{8}{|c|}{ Bus Participation Factors, $($ Load Factor $=1)$} \\
\hline & $\lambda_{2 \mathbf{z}}$ & $\lambda_{2 g}$ & $\lambda_{2 \star}$ & $\lambda_{25}$ & $\lambda_{2 \mathrm{\epsilon}}$ & $\lambda_{27}$ & $\lambda_{2 \mathrm{~g}}$ & $\lambda_{29}$ \\
\hline 1 & 0.00139 & & 0.06536 & & & & & \\
\hline 2 & & & & & & & & \\
\hline 3 & & & & & & 07 & & \\
\hline 4 & & & 0.01063 & & & 0.00037 & & \\
\hline 5 & & & 0 & & & 18 & & \\
\hline 6 & & & & 00 & & 61 & & \\
\hline 7 & & & & & & & & \\
\hline 8 & & & & 00 & & 55 & & \\
\hline 9 & & & & & & & & \\
\hline 10 & & & & 03 & & 40 & & \\
\hline 11 & & & & & & & & \\
\hline 12 & & & 0.0 & 17 & & 95 & & \\
\hline 13 & & & & & & & & \\
\hline 14 & & & & 12 & & 91 & & \\
\hline 15 & & & & & & & & \\
\hline 16 & & & & 0.00 & & & & \\
\hline 17 & & & & & & & & \\
\hline 18 & & & & 21 & & & & \\
\hline 19 & & & & & & 447 & & \\
\hline 20 & & & & & & & & \\
\hline 21 & & & & & & 0.00320 & & \\
\hline 22 & & & & & & & & \\
\hline 23 & & & & 0.03 & & 0.00928 & 0.00689 & 0.08402 \\
\hline 24 & & & & 0.00 & & & & 0.00016 \\
\hline 25 & & & & 0.00 & & 0.01215 & & 0.00170 \\
\hline 26 & & & & & & 0.06096 & 0.07134 & 0.01012 \\
\hline 27 & & & & & & 0.09353 & & \\
\hline 28 & & & 0.00 & 0.05 & & 0.01886 & 0.03069 & 0.00537 \\
\hline 29 & 0.00141 & & & & & 0.00767 & & \\
\hline
\end{tabular}




\begin{tabular}{|r|c|c|c|c|c|c|c|}
\hline \multirow{2}{*}{ Bus } & \multicolumn{7}{|c|}{ Bus Participation Factors, (Load Factor $=1.25)$} \\
\cline { 2 - 8 } & $\lambda_{\mathbf{2}}$ & $\lambda_{\mathbf{z}}$ & $\lambda_{\mathbf{3}}$ & $\lambda_{\mathbf{a}}$ & $\lambda_{\mathbf{5}}$ & $\lambda_{\mathbf{6}}$ & $\lambda_{\boldsymbol{z}}$ \\
\hline 1 & 0.00000 & 0.00000 & 0.00000 & 0.00000 & 0.00068 & 0.00006 & 0.00382 \\
\hline 2 & 0.00000 & 0.00000 & 0.00001 & 0.00001 & 0.10266 & 0.00803 & 0.45917 \\
\hline 3 & 0.00001 & 0.00001 & 0.00018 & 0.00006 & 0.11082 & 0.00620 & 0.04629 \\
\hline 4 & 0.00364 & 0.00101 & 0.00000 & 0.00160 & 0.01265 & 0.00001 & 0.00689 \\
\hline 5 & 0.38672 & 0.02275 & 0.00001 & 0.01450 & 0.00139 & 0.08300 & 0.00040 \\
\hline 6 & 0.53034 & 0.00576 & 0.00000 & 0.02084 & 0.00021 & 0.01101 & 0.00083 \\
\hline 7 & 0.02241 & 0.00154 & 0.00000 & 0.45597 & 0.00052 & 0.01378 & 0.00003 \\
\hline 8 & 0.01579 & 0.00225 & 0.00000 & 0.49238 & 0.00031 & 0.00001 & 0.00017 \\
\hline 9 & 0.00001 & 0.00000 & 0.00000 & 0.00202 & 0.00000 & 0.00000 & 0.00000 \\
\hline 10 & 0.00933 & 0.58208 & 0.00009 & 0.00108 & 0.00072 & 0.00031 & 0.00023 \\
\hline 11 & 0.03055 & 0.17901 & 0.00004 & 0.00515 & 0.01526 & 0.35635 & 0.00001 \\
\hline 12 & 0.00002 & 0.00071 & 0.00000 & 0.00000 & 0.00000 & 0.00000 & 0.00000 \\
\hline 13 & 0.00117 & 0.19744 & 0.00000 & 0.00507 & 0.01802 & 0.36350 & 0.00015 \\
\hline 14 & 0.00000 & 0.00729 & 0.00029 & 0.00128 & 0.01985 & 0.11707 & 0.00146 \\
\hline 15 & 0.00000 & 0.00006 & 0.03148 & 0.00002 & 0.00967 & 0.00173 & 0.00145 \\
\hline 16 & 0.00000 & 0.00006 & 0.73879 & 0.00000 & 0.02714 & 0.00274 & 0.00824 \\
\hline 17 & 0.00000 & 0.00000 & 0.08987 & 0.00001 & 0.33770 & 0.01554 & 0.14168 \\
\hline 18 & 0.00000 & 0.00000 & 0.00669 & 0.00001 & 0.26435 & 0.01393 & 0.03783 \\
\hline 19 & 0.00000 & 0.00000 & 0.00940 & 0.00000 & 0.00226 & 0.00025 & 0.00107 \\
\hline 20 & 0.00000 & 0.00000 & 0.00016 & 0.00000 & 0.00016 & 0.00002 & 0.00010 \\
\hline 21 & 0.00000 & 0.00000 & 0.01639 & 0.00000 & 0.00358 & 0.00041 & 0.00911 \\
\hline 22 & 0.00000 & 0.00000 & 0.00037 & 0.00000 & 0.00074 & 0.00010 & 0.03050 \\
\hline 23 & 0.00000 & 0.00000 & 0.00023 & 0.00000 & 0.00006 & 0.00000 & 0.00875 \\
\hline 24 & 0.00000 & 0.00001 & 0.10485 & 0.00000 & 0.02440 & 0.00268 & 0.00741 \\
\hline 25 & 0.00000 & 0.00000 & 0.00000 & 0.00000 & 0.02780 & 0.00238 & 0.20313 \\
\hline 26 & 0.00000 & 0.00000 & 0.00002 & 0.00000 & 0.00030 & 0.00000 & 0.01324 \\
\hline 27 & 0.00000 & 0.00000 & 0.00113 & 0.00000 & 0.01876 & 0.00089 & 0.01794 \\
\hline 28 & 0.00000 & 0.00000 & 0.00000 & 0.00000 & 0.00000 & 0.00000 & 0.00007 \\
\hline 29 & 0.00000 & 0.00000 & 0.00000 & 0.00000 & 0.00000 & 0.00000 & 0.00003 \\
\hline
\end{tabular}




\begin{tabular}{|r|c|c|c|c|c|c|c|}
\hline \multirow{2}{*}{ Bus } & \multicolumn{7}{|c|}{ Bus Participation Factors, (Load Factor $=1.25)$} \\
\cline { 2 - 8 } & $\lambda_{\mathbf{z}}$ & $\lambda_{\mathbf{g}}$ & $\lambda_{1 \mathbf{0}}$ & $\lambda_{1 \mathbf{1}}$ & $\lambda_{1 \mathbf{z}}$ & $\lambda_{1 \mathbf{z}}$ & $\lambda_{1 \mathbf{}}$ \\
\hline 1 & 0.00016 & 0.00025 & 0.00001 & 0.00068 & 0.00074 & 0.00024 & 0.00002 \\
\hline 2 & 0.01877 & 0.02173 & 0.00020 & 0.02379 & 0.02200 & 0.00705 & 0.00051 \\
\hline 3 & 0.00141 & 0.01040 & 0.00341 & 0.35618 & 0.00079 & 0.08824 & 0.00057 \\
\hline 4 & 0.00018 & 0.29653 & 0.00011 & 0.03269 & 0.01390 & 0.00097 & 0.00005 \\
\hline 5 & 0.00000 & 0.14049 & 0.00098 & 0.05844 & 0.05633 & 0.04543 & 0.00001 \\
\hline 6 & 0.00001 & 0.13105 & 0.00075 & 0.03771 & 0.04922 & 0.04927 & 0.00002 \\
\hline 7 & 0.00000 & 0.02287 & 0.00044 & 0.02778 & 0.03801 & 0.03590 & 0.00001 \\
\hline 8 & 0.00000 & 0.02980 & 0.00043 & 0.02529 & 0.03875 & 0.03923 & 0.00001 \\
\hline 9 & 0.00000 & 0.00037 & 0.00001 & 0.00074 & 0.00137 & 0.00146 & 0.00000 \\
\hline 10 & 0.00000 & 0.04577 & 0.00008 & 0.00697 & 0.00006 & 0.00423 & 0.00002 \\
\hline 11 & 0.00001 & 0.01503 & 0.00009 & 0.00095 & 0.03496 & 0.07044 & 0.00009 \\
\hline 12 & 0.00000 & 0.00018 & 0.00000 & 0.00013 & 0.00000 & 0.00011 & 0.00000 \\
\hline 13 & 0.00002 & 0.00203 & 0.00038 & 0.01667 & 0.03161 & 0.03413 & 0.00001 \\
\hline 14 & 0.00003 & 0.23779 & 0.00000 & 0.00165 & 0.06492 & 0.16242 & 0.00027 \\
\hline 15 & 0.00019 & 0.01153 & 0.00066 & 0.00154 & 0.03291 & 0.09313 & 0.00020 \\
\hline 16 & 0.00085 & 0.00145 & 0.00039 & 0.00014 & 0.00000 & 0.00000 & 0.00000 \\
\hline 17 & 0.00747 & 0.00849 & 0.00067 & 0.09375 & 0.00119 & 0.03307 & 0.00021 \\
\hline 18 & 0.00261 & 0.00109 & 0.00152 & 0.09061 & 0.03849 & 0.00000 & 0.00046 \\
\hline 19 & 0.00011 & 0.00040 & 0.67114 & 0.00302 & 0.00000 & 0.00000 & 0.00000 \\
\hline 20 & 0.00001 & 0.00006 & 0.28603 & 0.00148 & 0.00000 & 0.00000 & 0.00000 \\
\hline 21 & 0.07320 & 0.00037 & 0.00181 & 0.00149 & 0.00077 & 0.00020 & 0.00000 \\
\hline 22 & 0.60702 & 0.00002 & 0.00074 & 0.00077 & 0.00059 & 0.00017 & 0.00000 \\
\hline 23 & 0.25949 & 0.00014 & 0.00115 & 0.00114 & 0.00082 & 0.00022 & 0.00000 \\
\hline 24 & 0.01835 & 0.00428 & 0.02770 & 0.02478 & 0.01517 & 0.00409 & 0.00000 \\
\hline 25 & 0.00855 & 0.01708 & 0.00109 & 0.10376 & 0.00827 & 0.05471 & 0.00081 \\
\hline 26 & 0.00062 & 0.00024 & 0.00000 & 0.01275 & 0.31865 & 0.19315 & 0.00673 \\
\hline 27 & 0.00093 & 0.00058 & 0.00022 & 0.07471 & 0.21475 & 0.06790 & 0.00496 \\
\hline 28 & 0.00000 & 0.00000 & 0.00000 & 0.00007 & 0.00120 & 0.00015 & 0.31522 \\
\hline 29 & 0.00000 & 0.00000 & 0.00000 & 0.00033 & 0.01450 & 0.01410 & 0.66982 \\
\hline
\end{tabular}




\begin{tabular}{|r|c|c|c|c|c|c|c|}
\hline \multirow{2}{*}{ Bus } & \multicolumn{7}{|c|}{ Bus Participation Factors, (Load Factor $=1.25)$} \\
\cline { 2 - 8 } & $\lambda_{1 \mathbf{s}}$ & $\lambda_{1 \mathbf{6}}$ & $\lambda_{1 \mathbf{7}}$ & $\lambda_{1 \mathbf{s}}$ & $\lambda_{1 \mathbf{g}}$ & $\lambda_{2 \mathbf{0}}$ & $\lambda_{2 \mathbf{1}}$ \\
\hline 1 & 0.00002 & 0.00000 & 0.00134 & 0.00482 & 0.00321 & 0.02872 & 0.00054 \\
\hline 2 & 0.00032 & 0.00001 & 0.00707 & 0.02724 & 0.01159 & 0.07785 & 0.00103 \\
\hline 3 & 0.00038 & 0.00002 & 0.02951 & 0.00740 & 0.02041 & 0.00654 & 0.00175 \\
\hline 4 & 0.12714 & 0.00055 & 0.06549 & 0.32048 & 0.00179 & 0.00100 & 0.00045 \\
\hline 5 & 0.01702 & 0.00003 & 0.06804 & 0.00452 & 0.02262 & 0.00017 & 0.00290 \\
\hline 6 & 0.00688 & 0.00000 & 0.06384 & 0.00164 & 0.02430 & 0.00020 & 0.00320 \\
\hline 7 & 0.02214 & 0.00005 & 0.07472 & 0.00176 & 0.03575 & 0.00918 & 0.00751 \\
\hline 8 & 0.01847 & 0.00003 & 0.07192 & 0.00023 & 0.03430 & 0.00933 & 0.00748 \\
\hline 9 & 0.00122 & 0.00000 & 0.01418 & 0.00003 & 0.00998 & 0.00355 & 0.00421 \\
\hline 10 & 0.00019 & 0.00001 & 0.05667 & 0.11056 & 0.01797 & 0.04304 & 0.00025 \\
\hline 11 & 0.00125 & 0.00001 & 0.06511 & 0.11084 & 0.02503 & 0.03392 & 0.00134 \\
\hline 12 & 0.00001 & 0.00000 & 0.11689 & 0.01609 & 0.07778 & 0.01120 & 0.00755 \\
\hline 13 & 0.00307 & 0.00001 & 0.06602 & 0.03943 & 0.01532 & 0.02734 & 0.00002 \\
\hline 14 & 0.00438 & 0.00001 & 0.06574 & 0.10083 & 0.00149 & 0.00473 & 0.00512 \\
\hline 15 & 0.27987 & 0.00083 & 0.04023 & 0.00319 & 0.02604 & 0.20023 & 0.05747 \\
\hline 16 & 0.02189 & 0.00000 & 0.02383 & 0.01556 & 0.04365 & 0.01509 & 0.05155 \\
\hline 17 & 0.00308 & 0.00004 & 0.02913 & 0.01489 & 0.07393 & 0.04145 & 0.01237 \\
\hline 18 & 0.03692 & 0.00008 & 0.03224 & 0.01522 & 0.06123 & 0.12588 & 0.01039 \\
\hline 19 & 0.00430 & 0.00000 & 0.00382 & 0.00006 & 0.00852 & 0.00047 & 0.01463 \\
\hline 20 & 0.00944 & 0.00000 & 0.00143 & 0.00775 & 0.00381 & 0.01361 & 0.00865 \\
\hline 21 & 0.00485 & 0.58648 & 0.01504 & 0.00005 & 0.03674 & 0.00174 & 0.07463 \\
\hline 22 & 0.03007 & 0.00198 & 0.00490 & 0.01574 & 0.01379 & 0.02132 & 0.03594 \\
\hline 23 & 0.03227 & 0.31243 & 0.00529 & 0.02185 & 0.01501 & 0.02683 & 0.03962 \\
\hline 24 & 0.37158 & 0.09736 & 0.02193 & 0.05328 & 0.04628 & 0.03093 & 0.06954 \\
\hline 25 & 0.00083 & 0.00004 & 0.00570 & 0.07215 & 0.01825 & 0.22491 & 0.00788 \\
\hline 26 & 0.00229 & 0.00002 & 0.01538 & 0.00976 & 0.10350 & 0.01866 & 0.09035 \\
\hline 27 & 0.00001 & 0.00001 & 0.02503 & 0.02358 & 0.12703 & 0.01861 & 0.02370 \\
\hline 28 & 0.00012 & 0.00000 & 0.00647 & 0.00078 & 0.08268 & 0.00260 & 0.31734 \\
\hline 29 & 0.00001 & 0.00000 & 0.00305 & 0.00026 & 0.03798 & 0.00089 & 0.14262 \\
\hline
\end{tabular}




\begin{tabular}{|c|c|c|c|c|c|c|c|c|}
\hline \multirow[b]{2}{*}{ Bus } & \multicolumn{8}{|c|}{ Bus Participation Factors, $($ Load Factor $=1.25)$} \\
\hline & $\lambda_{22}$ & $\lambda_{2 \mathrm{z}}$ & $\lambda_{24}$ & $\lambda_{25}$ & $\lambda_{26}$ & $\lambda_{2 \bar{\gamma}}$ & $\lambda_{2 g}$ & $\lambda_{29}$ \\
\hline 1 & 0.00052 & 0.17573 & 0.07246 & 0.62278 & 0.00343 & 0.06113 & 0.01827 & 0.00036 \\
\hline 2 & 0.00081 & 0.08226 & 0.08275 & 0.02043 & 0.00017 & 0.02187 & 0.00262 & 0.00003 \\
\hline 3 & 0.00339 & 0.09897 & 0.02037 & 0.00087 & 0.00312 & 0.14529 & 0.02998 & 0.00745 \\
\hline 4 & 0.01084 & 0.01070 & 0.00980 & 0.00265 & 0.05126 & 0.00478 & 0.01739 & 0.00547 \\
\hline 5 & 0.04406 & 0.00035 & 0.00067 & 0.00021 & 0.00240 & 0.00472 & 0.01164 & 0.01019 \\
\hline 6 & 0.03285 & 0.00079 & 0.00048 & 0.00017 & 0.00199 & 0.00159 & 0.01365 & 0.01139 \\
\hline 7 & 0.10487 & 0.00507 & 0.01798 & 0.00020 & 0.00795 & 0.01788 & 0.04635 & 0.02934 \\
\hline 8 & 0.11090 & 0.00546 & 0.01543 & 0.00054 & 0.01630 & 0.01510 & 0.03153 & 0.01855 \\
\hline 9 & 0.07621 & 0.01276 & 0.01361 & 0.02226 & 0.41342 & 0.04036 & 0.19644 & 0.18577 \\
\hline 10 & 0.00672 & 0.00003 & 0.01927 & 0.00153 & 0.03675 & 0.05135 & 0.00369 & 0.00100 \\
\hline 11 & 0.00181 & 0.00017 & 0.01009 & 0.00087 & 0.01834 & 0.02280 & 0.00002 & 0.00046 \\
\hline 12 & 0.58220 & 0.00056 & 0.00875 & 0.00475 & 0.12703 & 0.04074 & 0.00405 & 0.00126 \\
\hline 13 & 0.01538 & 0.00000 & 0.02041 & 0.00203 & 0.05316 & 0.06738 & 0.01374 & 0.00649 \\
\hline 14 & 0.00088 & 0.00021 & 0.00339 & 0.00296 & 0.09048 & 0.04362 & 0.03725 & 0.02462 \\
\hline 15 & 0.00075 & 0.01484 & 0.09932 & 0.00000 & 0.02058 & 0.02659 & 0.01206 & 0.03342 \\
\hline 16 & 0.00026 & 0.01350 & 0.03092 & 0.00045 & 0.00012 & 0.00010 & 0.00009 & 0.00318 \\
\hline 17 & 0.00002 & 0.01292 & 0.04269 & 0.02047 & 0.00880 & 0.00952 & 0.00037 & 0.00068 \\
\hline 18 & 0.00090 & 0.06525 & 0.02037 & 0.02165 & 0.00291 & 0.12658 & 0.01646 & 0.00632 \\
\hline 19 & 0.00008 & 0.00813 & 0.01172 & 0.00081 & 0.00013 & 0.00083 & 0.14143 & 0.11742 \\
\hline 20 & 0.00005 & 0.00744 & 0.07402 & 0.00110 & 0.00017 & 0.00253 & 0.33176 & 0.25024 \\
\hline 21 & 0.00043 & 0.06692 & 0.02018 & 0.03088 & 0.00351 & 0.00037 & 0.00098 & 0.04988 \\
\hline 22 & 0.00022 & 0.04760 & 0.07663 & 0.03104 & 0.00327 & 0.00073 & 0.00154 & 0.07421 \\
\hline 23 & 0.00024 & 0.05350 & 0.09274 & 0.03548 & 0.00372 & 0.00086 & 0.00180 & 0.08634 \\
\hline 24 & 0.00037 & 0.03080 & 0.03906 & 0.00432 & 0.00067 & 0.00006 & 0.00001 & 0.00007 \\
\hline 25 & 0.00025 & 0.06351 & 0.16578 & 0.00747 & 0.00069 & 0.00321 & 0.00007 & 0.00167 \\
\hline 26 & 0.00048 & 0.00709 & 0.02048 & 0.02309 & 0.02036 & 0.09924 & 0.02026 & 0.02335 \\
\hline 27 & 0.00020 & 0.02671 & 0.00416 & 0.08869 & 0.05671 & 0.13922 & 0.03203 & 0.03155 \\
\hline 28 & 0.00297 & 0.13138 & 0.00472 & 0.03669 & 0.03683 & 0.03672 & 0.01030 & 0.01368 \\
\hline 29 & 0.00132 & 0.05733 & 0.00175 & 0.01563 & 0.01574 & 0.01481 & 0.00421 & 0.00562 \\
\hline
\end{tabular}

